Florida International University FIU Digital Commons

4-2-1998

\title{
Crack tip stress study for elastic-perfectly plastic materials with some applications
}

Ivan Enrique Esparragoza

Florida International University

DOI: $10.25148 /$ etd.FI15101313

Follow this and additional works at: https://digitalcommons.fiu.edu/etd

Part of the Mechanical Engineering Commons

\section{Recommended Citation}

Esparragoza, Ivan Enrique, "Crack tip stress study for elastic-perfectly plastic materials with some applications" (1998). FIU Electronic Theses and Dissertations. 3242.

https://digitalcommons.fiu.edu/etd/3242

This work is brought to you for free and open access by the University Graduate School at FIU Digital Commons. It has been accepted for inclusion in FIU Electronic Theses and Dissertations by an authorized administrator of FIU Digital Commons. For more information, please contact dcc@fiu.edu. 
FLORIDA INTERNATIONAL UNIVERSITY

Miami, Florida

CRACK TIP STRESS STUDY

FOR ELASTIC-PERFECTLY PLASTIC MATERIALS

WITH SOME APPLICATIONS

A dissertation submitted in partial satisfaction of the requirements for the degree of

DOCTOR OF PHILOSOPHY

IN

MECHANICAL ENGINEERING

by

Ivan Enrique Esparragoza 
To: Dean Gordon Hopkins

College of Engineering and Design.

This dissertation, written by Ivan Enrique Esparragoza, and entitled Crack Tip Stress Study for Elastic-Perfectly Plastic Materials with Some Applications, having been approved in respect to style and intellectual content, is referred to you for judgement.

We have read this dissertation and recommend that it be approved.

Norman Munroe

Ibrahim Tansel

Sabri Tosunoglu

Genady P. Cherepanov

Major Professor

Date of defense: April 2nd, 1998

The dissertation of Ivan Enrique Esparragoza is approved

Dean Gordon Hopkins

College of Engineering and Design

Dr. Richard L. Campbell

Dean Of Graduate Studies

Florida International University, 1998 
To my mother, my family and to my future wife Haydee. 


\section{ACKNOWLEDGMENTS}

I would like to express my gratitude to all the people who helped me to complete my Ph.D. program and this dissertation. To mention all of them by the names would be a long task and I would not like to leave anybody out from this list. Therefore, thanks to all my classmates, students, professors, members of the Mechanical Engineering Department and everybody who, in one or other way, helped me to reach this goal.

I wish to thank the members of my committee for their support and their helpful comments.

I am deeply grateful with Dr. Genady P. Cherepanov for his invaluable support, guidance and encouraging comments throughout the Ph.D. program, and especially for having the confidence in me to give me the opportunity to work with him in this project and during my whole stay as a research assistant in the department. It has been my privilege to discuss with him novel topics in applied mechanics. It has been an honor for me to work with Dr. Cherepanov and I look forward to collaborating with him in future research work.

I would like to express my sincere appreciation to Dr. Richard Irey, chairman of the Department of Mechanical Engineering, for his support, amiability and for giving me the opportunity to work as an instructor in the department.

Finally, I would like to say thanks to my mother, family, friends and my lovely Wary. Without their patience, understanding, support, and most of all love, the completion of this work would have not been possible. 


\title{
ABSTRACT OF THE DISSERTATION
}

\author{
CRACK TIP STRESS STUDY \\ FOR ELASTIC-PERFECTLY PLASTIC MATERIALS \\ WITH SOME APPLICATIONS \\ by \\ Ivan Enrique Esparragoza \\ Florida International University, 1998 \\ Miami, Florida \\ Professor Genady P. Cherepanov, Major Professor
}

The problems of plasticity and non-linear fracture mechanics have been generally recognized as the most difficult problems of solid mechanics. The present dissertation is devoted to some problems on the intersection of both plasticity and non-linear fracture mechanics. The crack tip is responsible for the crack growth and therefore is the focus of fracture science. The problem of crack tip has been studied by an army of outstanding scholars and engineers in this century, but has not, as yet, been solved for many important practical situations. The aim of this investigation is to provide an analytical solution to the problem of plasticity at the crack tip for elastic-perfectly plastic materials and to apply the solution to a classical problems of the mechanics of composite materials. 
In this work, the stresses inside the plastic region near the crack tip in a composite material made of two different elastic-perfectly plastic materials are studied. The problems of an interface crack, a crack impinging an interface at the right angle and at arbitrary angles are examined. The constituent materials are assumed to obey the Huber-Mises yielding condition criterion. The theory of slip lines for plane strain is utilized. For the particular homogeneous case these problems have two solutions: the continuous solution found earlier by Prandtl and modified by Hill and Sokolovsky, and the discontinuous solution found later by Cherepanov. The same type of solutions were discovered in the inhomogeneous problems of the present study. Some reasons to prefer the discontinuous solution are provided. The method is also applied to the analysis of a contact problem and a push-in/pull-out problem to determine the critical load for plasticity in these classical problems of the mechanics of composite materials.

The results of this dissertation published in three journal articles (two of which are under revision) will also be presented in the Invited Lecture at the $7^{\text {th }}$ International Conference on Plasticity (Cancun, Mexico, January 1999). 
CHAPTER

\section{CHAPTER 1. INTRODUCTION}

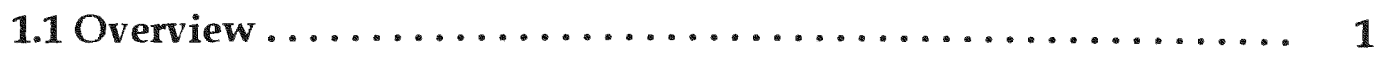

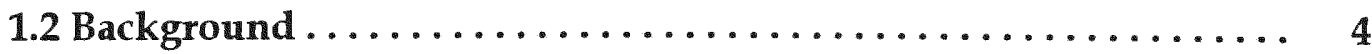

1.3 Objective and Significance $\ldots \ldots \ldots \ldots \ldots \ldots \ldots \ldots \ldots, 8$

CHAPTER 2. PLANE STRAIN SLIP LINE THEORY

2.1 Introduction .............................. 10

2.2 Fundamental Equations for Plane Strain Problem......... 11

2.3 Shear Lines or Slip Lines . . . . . . . . . . . . . . . . . 14

2.4 Hencky Stress Equations....................... 18

2.5 Geringer Velocity Equations ................... 20

CHAPTER 3. AN INTERFACE CRACK

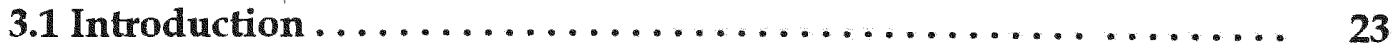

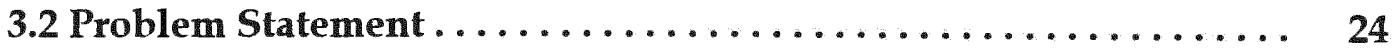

3.3 Continuous Solution ........................ 29

3.4 Discontinuous Solution ..................... 36

3.5 Velocity Distribution $\ldots \ldots \ldots \ldots \ldots \ldots \ldots \ldots \ldots \ldots, 41$

CHAPTER 4. A CRACK IMPINGING THE INTERFACE AT A RIGHT ANGLE

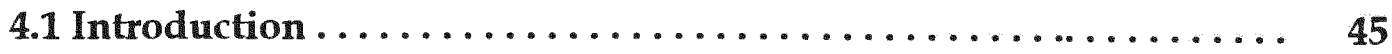

4.2 Problem Statement . . . . . . . . . . . . . . . . . . 46

4.3 Continuous Solution ....................... 51

4.4 Discontinuous Solution ...................... 55

4.5 Velocity Distribution $\ldots \ldots \ldots \ldots \ldots \ldots \ldots \ldots \ldots \ldots \ldots \ldots \ldots$

\section{CHAPTER 5. A CRACK IMPINGING THE INTERFACE AT AN} ARBITRARY ANGLE

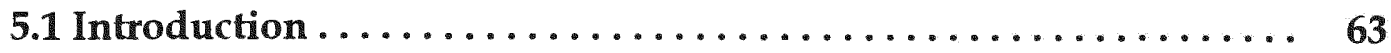

5.2 Problem Statement ............................ 64

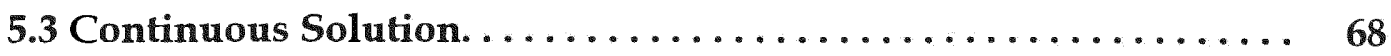

5.4 Discontinuous Solution. ...................... 70

5.5 Velocity Distribution $\ldots \ldots \ldots \ldots \ldots \ldots \ldots \ldots \ldots \ldots \ldots \ldots$ 
CHAPTER 6. PROBLEM OF SMOOTH RIGID PUNCH

6.1 Introduction ............................ 80

6.2 Problem Statement .......................... 80

6.3 Continuous Solution ......................... 82

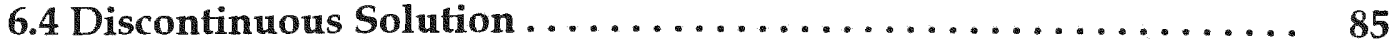

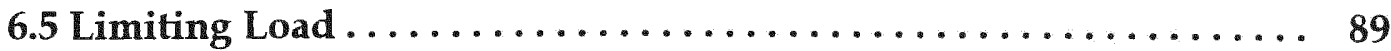

6.6 Velocity Distribution ........................ 90

CHAPTER 7. THE PUSH-IN AND PULL-OUT PROBLEMS

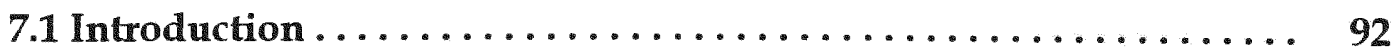

7.2 Some Results of Fiber Stress Analysis ............... 93

7.3 Statement of the Plasticity Problem ................ 98

7.4 Continuous Solution ........................ 100

7.5 Discontinuous Solution . . . . . . . . . . . . . . . 103

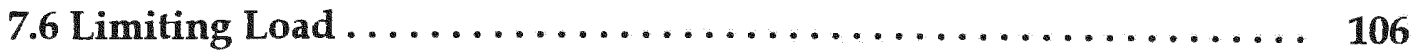

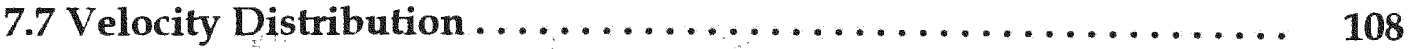

CHAPTER 8. DISCUSSION AND CONCLUSIONS

8.1 Introduction .................................. 109

8.2 The Plasticity Problem at the Crack Tip for an Inhomogeneous

Materials ............................. 110

8.3 The Push-in and Pull-out Problem .................. 113

8.4 Final Remarks and Recommendations .............. 116

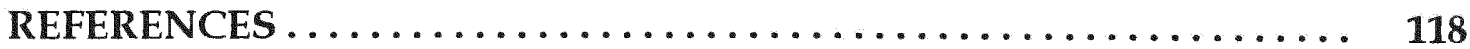

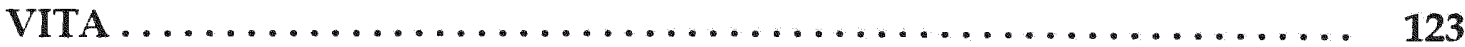




\section{LIST OF FIGURES}

Fig. 2.1 Principal stress directions and the $\alpha$ and $\beta$ directions at a point in a plastically deforming region. ................ 14

Fig. 3.1 The interface crack tip between two perfectly plastic materials: the geometry of characteristic lines in the crack tip

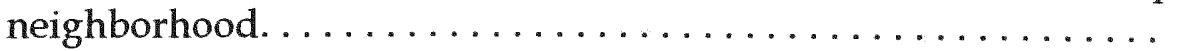

Fig. 3.2 Graph $\theta_{1}$ vs. $\lambda=\tau_{1} / \tau_{2}$ for the continuous solution of the inhomogeneous problem, Eq. (3.24).

Fig 3.3 Graph $\theta_{2}$ vs. $\lambda=\tau_{1} / \tau_{2}$ where $\theta_{2}=\frac{1}{2} \cos ^{-1}\left[-\lambda \cos 2 \theta_{1}\right]$ for the continuous solution of the inhomogeneous problem, Eq. (3.24).

Fig. 3.4 Graph $\frac{\sigma_{y}}{\tau_{1}} \quad$ vs. $\lambda=\frac{\tau_{1}}{\tau_{2}}$ for $0.71 \leq \lambda \leq 1$. The $\sigma_{y}$ is the corresponding stress for $\theta_{1}>\theta>-\theta_{2} \ldots \ldots \ldots \ldots \ldots \ldots \ldots$

Fig. 3.5 Graph $\frac{\tau_{x y}}{\tau_{1}} \quad$ vs. $\lambda=\frac{\tau_{1}}{\tau_{2}}$ for $0.71 \leq \lambda \leq 1$. The $\tau_{x y}$ is the corresponding stress for $\theta_{1}>\theta>-\theta_{2} \ldots \ldots \ldots \ldots \ldots \ldots$

Fig. 3.6 Graph $\frac{\sigma_{x}^{+}}{\tau_{1}}$ and $\frac{\sigma_{x}^{-}}{\tau_{1}}$ vs. $\lambda=\frac{\tau_{1}}{\tau_{2}}$ for $0.71 \leq \lambda \leq 1$. The $\sigma_{x}^{+}$is the corresponding stress for $\theta_{1}>\theta>0$, and the $\sigma_{x}^{-}$is the corresponding stress for $0>\theta>-\theta_{2} \ldots \ldots \ldots \ldots \ldots \ldots \ldots$

Fig. 3.7a Characteristic lines for the first solution of the plasticity theory. $\sigma_{y}=\tau_{1}(2+\pi), \sigma_{x}=\pi \tau_{1}, \tau_{x y}=0$ when $|\theta|<\frac{\pi}{4} \ldots \ldots \ldots \ldots \ldots$

Fig. 3.7b Characteristic lines for the second solution of the plasticity theory. $\sigma_{y}=4 \tau_{1}, \sigma_{x}=2 \tau_{1}, \tau_{x y}=0$ when $\theta_{1}=\theta_{2}=\frac{\pi}{2} \ldots \ldots \ldots$

Fig. 4.1 The crack impinging the interface of two elastic-perfectly plastic materials bonded along $x=0$. 
Fig. 4.2a Characteristic lines for the first solution of the plasticity theory. $\sigma_{y}=\tau_{1}(2+\pi), \sigma_{x}=\pi \tau_{1}, \tau_{x y}=0$ when $|\theta|<\frac{\pi}{4} \ldots \ldots \ldots \ldots \ldots$

Fig. 4.2b Characteristic lines for the second solution of the plasticity theory. $\sigma_{y}=4 \tau_{1}, \sigma_{x}=2 \tau_{1}, \tau_{x y}=0$ when $\theta_{1}=\theta_{2}=\frac{\pi}{2} \ldots \ldots \ldots$

Fig. 5.1 The crack impinging the interface of two elastic-perfectly plastic

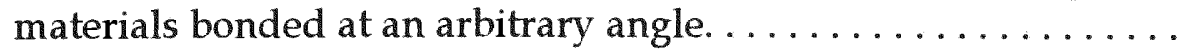

Fig. 5.2 The crack impinging an interface of two different elastic-plastic solids under an arbitrary angle $\varphi$ : the case when $\pi / 4 \leq \varphi \leq \pi / 2$

Fig 5.3 Graph $\frac{\sigma_{y}}{\tau_{2}}$ vs. $\lambda$ for discontinuous solution in the case of a crack impinging an interface at an arbitrary angle. The $\sigma_{y}$ is the corresponding stress for $\theta_{2}>\theta>0 \ldots \ldots \ldots \ldots \ldots \ldots \ldots$

Fig 5.4 Graph $\frac{\sigma_{x}}{\tau_{2}}$ vs. $\lambda$ for discontinuous solution in the case of a crack impinging an interface at an arbitrary angle. The $\sigma_{x}$ is the corresponding stress for $\theta_{2}>\theta>0 \ldots \ldots \ldots \ldots \ldots \ldots \ldots$

Fig. 6.1 The smooth rigid punch problem for an inhomogeneous

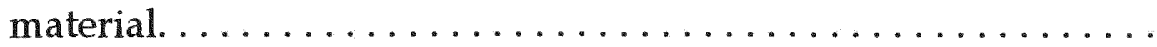

Fig. 7.1 The axisymmetric problem of pull-out of a foregoing elastic fiber embedded in a softer elastic matrix. The cylindrical domain of non-zero perturbed field. . . . . . . . . . . . . . . . . .

Fig. 7.2 The plasticity region in the push-in or pull-out problem. . . . . . 99 


\section{LIST OF TABLES}

Table 3.1 Numerical solution of the equation system (3.24) when

Page

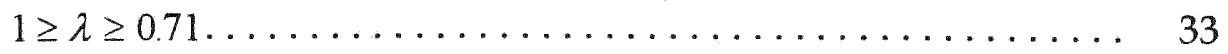

Table $5.1 \quad$ Numerical solution of equation $(5.20) \ldots \ldots \ldots \ldots \ldots \ldots$

Table 5.2 Numerical solution for $\theta_{2} \ldots \ldots \ldots \ldots \ldots \ldots \ldots \ldots \ldots$

Table 5.3a Numerical solution of the equation system (5.24) for $\frac{\sigma_{y}}{\tau_{2}} \ldots \ldots 75$

Table 5.3b Numerical solution of the equation system (5.24) for $\frac{\sigma_{x}}{\tau_{2}} \ldots \ldots 76$ 


\section{LIST OF SYMBOLS}

$\begin{array}{ll}K_{c} & \text { Fracture toughness } \\ \Gamma & \text { Invariant } \Gamma \text {-integral } \\ Y & \text { Yield stress } \\ K & \text { Stress intensity factor } \\ k & \text { Yield shear stress } \\ x, y, z & \text { Cartesian coordinates } \\ \sigma_{y}, \sigma_{x} & \text { Normal stresses in Cartesian coordinates } \\ \tau_{x y} & \text { Shear stress in Cartesian coordinates } \\ p & \text { Hydrostatic pressure } \\ \varphi & \text { Angle of first principal direction } \\ \sigma_{1}, \sigma_{2} \sigma_{3} & \text { Principal stresses } \\ \alpha, \beta & \text { Shear directions } \\ \theta & \text { Angle of first shear direction } \\ v_{\alpha}, v_{\beta} & \text { Velocity along } \alpha \text { and } \beta \text { directions } \\ v_{x}, v_{y} & \text { Velocity components in Cartesian coordinates } \\ \tau_{1} & \text { Yield shear stress for material \#1 } \\ \tau_{2} & \text { Yield shear stress for material \#2 } \\ \Delta & \text { Region defined by three points } \\ r, \theta & \text { Polar coordinates } \\ \sigma_{r}, \sigma_{\theta} & \text { Stresses in polar coordinates } \\ \tau_{r \theta} & \text { Shear stress in polar coordinates } \\ f(\theta) & \text { Arbitrary function of } \theta \\ C_{1}, C_{2}, C_{3} & \text { Arbitrary constants } \\ \lambda & \end{array}$




\begin{tabular}{|c|c|}
\hline$v_{r}, v_{\theta}$ & Velocity components in polar coordinates \\
\hline$V_{0}$ & Undetermined constant velocity \\
\hline$\varphi$ & Angle of incidence of the crack into the interface \\
\hline$F$ & Force \\
\hline$r_{0}$ & Radius of the fiber \\
\hline$l$ & Embedded length of the fiber \\
\hline$E_{f}$ & Young's modulus of the fiber \\
\hline$E_{m}$ & Young's modulus of the matrix \\
\hline$v_{f}$ & Poisson's ratio of the fiber \\
\hline$v_{m}$ & Poisson's ratio of the matrix \\
\hline$\xi$ & Dimensionless relation equals to $r_{0} / l$ \\
\hline$\varepsilon$ & Dimensionless relation equals to $E_{m} / E_{f}$ \\
\hline$\sigma_{z}$ & Normal stress in the $z$ direction \\
\hline$\tau_{r z}$ & Shear stress in the $r z$ direction \\
\hline$W, w$ & Displacement in $z$ direction \\
\hline$r_{*}$ & Radius of perturbation domain in the matrix \\
\hline$\eta$ & Fitting index \\
\hline $\sinh$ & Hyperbolic sine \\
\hline $\cosh$ & Hyperbolic cosine \\
\hline $\tanh$ & Hyperbolic tangent \\
\hline$\kappa$ & Expresion defined in Eq. (7.5) \\
\hline
\end{tabular}


$\sigma_{0} \quad$ Normal stress acting on the top of the fiber

$l_{D} \quad$ Debonding length

$\tau_{s} \quad$ Limiting shear stress at the interface 


\section{CHAPTER 1}

\section{INTRODUCTION}

\subsection{Overview}

Composite materials are different from conventional engineering material of the past. In fact, composites are two or more materials combined on a macroscopic scale to form a new useful material that exhibits the best qualities of their constituents and often some qualities that neither constituent material possesses. Usually, most engineering materials are homogeneous and isotropic; meanwhile, composites are often both inhomogeneous and anisotropic.

Generally speaking, composites are studied from a macromechanics point of view. Therefore, the composite is presumed to be a new homogeneous material, and the effects of the constituent materials are detected only as averaged apparent properties of the composite. On the other hand, micromechanics analysis studies the composite behavior wherein the interaction of constituent materials is examined on a microscopic scale.

The mechanics of failure of composite materials is a subject in the early stages of development. Although these materials have become very important in the new technology due to the improvement of some properties such as strength, 
stiffness, fatigue life and others, there are no generally recognized theories for the mechanics of failure.

Nevertheless, the presence of cracks in a composite material is almost impossible to avoid. They appear as a result of the fabrication process or due to the load applied to the composite and the interaction between the constituent materials. The most frequent cracks are those that appear along the interface or perpendicular to the interface of the two different materials. Now, it is well known from the theory of fracture mechanics that the stresses become infinite at the crack tip. Since this cannot occur in reality, plastic deformation takes place at the crack tip and the stresses remain finite. The development of plastic zones at the crack tip depends on the properties of each material among other factors. This phenomenon in inhomogeneous materials is complex and poorly understood and studied.

The theory of plasticity is still a young science and its application to the micromechanical behavior of inhomogeneous materials is limited. It is well understood that due to the high stress and strain concentrations which exists in local regions like the crack tip, the elastic limit of the constituent materials at these local regions might be exceeded before the macromechanics behavior of the composite material exhibits a nonlinearity. Therefore, a plastic region appears near the crack tip. This localized plastic behavior has a significant 
influence on the redistribution of stresses within the composite. The crack tip is responsible for the crack growth and therefore is the focus of fracture science. The problem of crack tip has been studies by an army of outstanding scholars and engineers in this century, but has not, as yet, been solved for many practical solutions.

Consequently, in this investigation attention will be focused in the distribution of stresses inside the plastic zone at the crack tip of an inhomogeneous material. This analysis is carried out at a micromechanical level where the response of the constituent materials is of importance. Additionally, the idea developed in this work is extended to the classic punch problem in the case of an inhomogeneous material.

Since the problems of plasticity and non-linear fracture mechanics have been generally recognized as most difficult problems of solid mechanics, in the context of the mechanics of materials, this type of investigation is conducive to a better understanding of the behavior of the inhomogeneous material in the presence of a crack, and the development of a theory to close the gap existing in the analysis of stresses in the plastic region at the crack tip for the case of composite materials. 


\subsection{Background}

The first yield criterion is due to Tresca who in 1864 published a preliminary account of experiments on punching and extrusion. His conclusions led to the formulation of a yield criterion which states that a metal yields plastically when the maximum shear stress attains a critical value. This was the first important investigation in this area for metals. This yield criterion was later used by SaintVenant (1870) [2] to determine the stresses in a partially plastic tube subjected to torsion or bending. The same year, Levy [3] proposed the three-dimensional relations between stress and plastic strain-rate following the ideas of SaintVenant. The method of linearisation for the plane strain problem was also introduced by Levy.

By the beginning of this century, Haar and Karman (1909) [4] obtained plasticity equations from a variational principle. Many experiments were performed during the next decade which were concerned with the yielding of tubes subjected to various stress states. From here various yield criteria were suggested. In 1904 Huber [5], and then in 1913, independently, von Mises [6] formulated a new yield criterion which was most satisfactory for the majority of metals. This criterion was later interpreted by Hencky as implying that yielding occurred when the elastic shear-strain energy attained a critical value. The 
Huber-Mises criterion is close to the Tresca criterion, but it better describes test data. Both coincide for plane strain.

In 1920 Prandtl showed that the plane plastic strain problem is hyperbolic and he determined the loads needed to indent a plane surface by a smooth flat punch. Hencky in 1923 produced the general theory applicable to the special solutions by Prandtl and also discovered the geometrical properties of the field of slip-lines for plane plastic strain. Later Geiringer (1930) obtained the velocity compatibility equations for flow along slip lines.

The correct approach to the solution of plane problem was clarified during the period 1945-1949. This period was characterized by a research stimulus due to the war. In 1950 Hill and Sokolovsky found the stresses in the plastic region at the crack tip for a homogeneous material. It was shown that these stresses coincide with those found previously by Prandtl at the punch edge and later modified by Hill (see also Prager and Hodge (1951)).

In the 1950s many authors contributed to the development of the plasticity theory. Green $(1951,53,54)$ presented a series of plane strain solutions; Prager (1953) introduced a simplification into slip line field solution; Alexander (1955) and Johnson (1956) presented some other contributions to plane strain slip line field solutions. 
Fracture mechanics is still a younger science than plasticity. Its main development has occurred after 1950. However, the basic idea of fracture mechanics was established in 1921 by Griffith. He stated that crack propagation will occur if the energy released upon crack growth is sufficient to provide all the energy that is required for crack growth. Minor progress was made in the next three decades. However, some important works can be cited. Mushkelishvili (1933) proposed a method for the solution to a plane problem for bodies containing either rectilinear or curvilinear cracks; Westergaard (1939) proposed a semi-reversed method of a solution to a plane problem for bodies containing rectilinear cracks; Irwin (1948) employed the Griffith criterion to a quasi-brittle fracture by adding the energy expended for plastic deformation of the crack fracture. The major contribution to the contemporary fracture mechanics was proposed by Irwin (1958). Considering the physical and mathematical concepts of Griffith and Westergaard he proposed that, for a given point, the coefficient of stress singularity at the incipience of both local fracture and the crack propagation is assumed to be a constant characteristic of the material. Then, the mechanical characteristic $K_{c}$ known as fracture toughness was introduced. In 1960 he formulated the length of the plastic zone appearing in the plastic correction at the crack tip. 
Other important contributions in the field of fracture mechanics and the plastic zone at the crack tip can be summarized as follows: Dugdale (1960) analyzed the length of the plastic zone ahead of the crack tip assuming the plastic zone thickness as zero; Wells (1961) introduced the plastic crack tip opening displacement as a measurement of a local plastic deformation at the crack tip as well as a fracture criterion in nonlinear fracture mechanics; Cherepanov (1967) proposed the invariant contour $\Gamma$-integral, and independently Rice (1968) the path independent J-integral, as a measurement of the elastic-plastic condition at the crack tip as well as a fracture criterion in the nonlinear fracture mechanics. Both $\Gamma$-integral and J-integral coincide in common situations.

The plasticity problem at the crack tip for hardening material was treated by Cherepanov (1967), Hutchinson (1968), and Rice and Rosengren (1968). In the 1970 's many other papers regarding the plastic crack problem were published. Among other authors it is important to mention Kahl and Reifsnider (1972), Goldman and Hutchinson (1975), Amazigo (1975, 1978), Shih and Hutchinson (1976), Shih (1976), McMeeking and Parks (1977), and Rice (1978).

Close to the topic under investigation, it is also important to include some works regarding the elastoplastic behavior of composites such as those by Marcal (1969), Adams (1970, 1973), Bert (1972), Repnau and Adams (1973). Other works 
regarding the mechanism of failure composites can be mentioned also such as those by Nair and Reifsnider (1974), Reifsnider et al (1976, 1977), Reifsnider (1977), and Stinchcomb and Reifsnider (1977).

A large list of references can be cited referent to the interaction between fiber and matrix in composite materials (see Cherepanov and Esparragoza (1995)). However, the study of the plasticity problem at the crack tip for the case of an inhomogeneous material is in its first step. The work by Cherepanov (1997) is probably the first attempt to solve a problem of this kind. In the present study special attention to this type of problems is the major objective. For this purpose, an extensive analysis is presented and some ideas are discussed to better understand this phenomenon.

\subsection{Objective and Significance}

The objectives of this investigation can be expressed as follows:

a. To study the stresses inside the plastic region at the crack tip for the case of an inhomogeneous material.

b. To show that in the homogeneous case there exist two solutions one of which is continuous and the other is discontinuous.

c. To analyze when each one of the solutions might be realized in practice since it is evident that both cannot be met in practice simultaneously. 
d. To extend this idea to estimate the load needed to indent a plane surface of an inhomogeneous material by a flat smooth punch.

e. To study the plasticity region in the push-out or pull-in problem.

The significance of this work is that this analysis provides new ideas directed to understand the mechanism of failure in an inhomogeneous material. This is also a motivation to encourage other scientists to continue working in the development of theories for the mechanics of failure of composite materials. A comparison of the theoretical results of the present work with experimental data would be a good topic for future research work. 


\section{CHAPTER 2}

\section{PLANE STRAIN SLIP LINE FIELD THEORY}

\subsection{Introduction}

In order to avoid mathematical difficulties in many of the problems of greatest practical interest, the elastic component of strain in the plastic region is disregarded. For consistency, the purely elastic strain in the non-plastic region is disregarded also. Therefore, in developing a field-type theory to provide information about the deformation from point to point in the plastically deforming region, strain hardening is usually neglected so that the material is assumed to flow at constant yield stress. This hypothetical material is referred to as rigid-perfectly plastic.

This chapter will be devoted to the behavior of a rigid-perfectly plastic material under conditions of plane strain, and in particular with certain general properties of the stress and velocity distribution in the plastic region.

A great deal of work has been done on solutions of this type of problem under conditions of plain strain, using the theory of slip lines. The theory has been shown to be very useful in many applications, despite its obvious limitations. 


\subsection{Fundamental Equations for Plane Strain Problem}

The plane strain model is appropriate when the flow is everywhere parallel to a given plane $(x, y)$ and the normal strain in the $z$ direction and the shear strains in $x z$ and $y z$ may be assumed to be zero. Here $x, y$, and $z$ are Cartesian coordinates. Since the shear stresses in the $x z$ and $y z$ directions are zero, then the normal stress in the $z$ direction is a principal stress.

The Huber-Mises yield criterion in terms of the components of the stress tensor is:

$$
\left(\sigma_{x}-\sigma_{y}\right)^{2}+\left(\sigma_{y}-\sigma_{z}\right)^{2}+\left(\sigma_{z}-\sigma_{x}\right)^{2}+6\left(\tau_{x y}^{2}+\tau_{y z}^{2}+\tau_{x x}^{2}\right)=6 k^{2}
$$

where $k$ is the yield stress in pure shear.

For plane strain deformation in the $x y$ plane:

$$
\begin{aligned}
& \sigma_{z}=\frac{1}{2}\left(\sigma_{x}+\sigma_{y}\right) \\
& \tau_{x z}=\tau_{y z}=0
\end{aligned}
$$

Therefore, from Eqs. (2.1) and (2.2) it can be found: 


$$
\frac{1}{4}\left(\sigma_{x}-\sigma_{y}\right)^{2}+\tau_{x y}^{2}=k^{2}
$$

The equilibrium equations to be satisfied are:

$$
\begin{aligned}
& \frac{\partial \sigma_{x}}{\partial x}+\frac{\partial \tau_{x y}}{\partial y}=0 \\
& \frac{\partial \sigma_{y}}{\partial y}+\frac{\partial \tau_{x y}}{\partial x}=0
\end{aligned}
$$

Equations (2.3) and (2.4) represent three equations in the three unknowns $\sigma_{x}, \sigma_{y}$ and $\tau_{x y}$. If the boundary conditions are given in terms of stresses, these equations are sufficient to give the stress distribution without any reference to the stress-strain relations. Such problems are called statically determined.

The principal stresses in the plastic region are:

$$
\begin{aligned}
& \sigma_{1}=\frac{1}{2}\left(\sigma_{x}+\sigma_{y}\right)+\left[\frac{1}{4}\left(\sigma_{x}-\sigma_{y}\right)^{2}+\tau_{x y}^{2}\right]^{1 / 2} \\
& \sigma_{2}=\sigma_{z}=\frac{1}{2}\left(\sigma_{x}+\sigma_{y}\right)=p \\
& \sigma_{3}=\frac{1}{2}\left(\sigma_{x}+\sigma_{y}\right)-\left[\frac{1}{4}\left(\sigma_{x}-\sigma_{y}\right)^{2}+\tau_{x y}^{2}\right]^{1 / 2}
\end{aligned}
$$


The maximum shear stress in the plane of flow is given by

$$
\tau_{\max }=\tau=\frac{1}{2}\left(\sigma_{1}-\sigma_{3}\right)=\left[\frac{1}{4}\left(\sigma_{x}-\sigma_{y}\right)^{2}+\tau_{x y}^{2}\right]^{1 / 2}
$$

Therefore, the principal stresses can be expressed as

$$
\sigma_{1}=p+k, \quad \sigma_{2}=\sigma_{z}=p, \quad \sigma_{3}=p-k
$$

Since the volume of an element of rigid-perfectly plastic material does not alter, each incremental distortion in a state of plane strain consists of a pure shear. Therefore, the sate of stresses at each point is a pure shear stress $k$ together with a hydrostatic pressure $p$.

If there is no work-hardening, and if the yielding is not influenced by hydrostatic pressure, $k$ must be constant and its value depends on the yield criterion.

There is no difference between the functional relation of stresses representing either the Huber-Mises or Tresca yield criteria under plane strain. 


\subsection{Shear Lines or Slip Lines}

The magnitudes of the principal stresses in the plastically deforming field are given by Eqs. (2.5) or, alternatively, by Eqs. (2.7) in terms of the hydrostatic pressure $p$ and the yield shear stress $k$. The first principal direction is defined as the direction of the algebraic maximum principal stress $\sigma_{1}$. Let $\phi$ be the angle between the first principal direction and the $x$ axis as shown in Fig. 2.1.

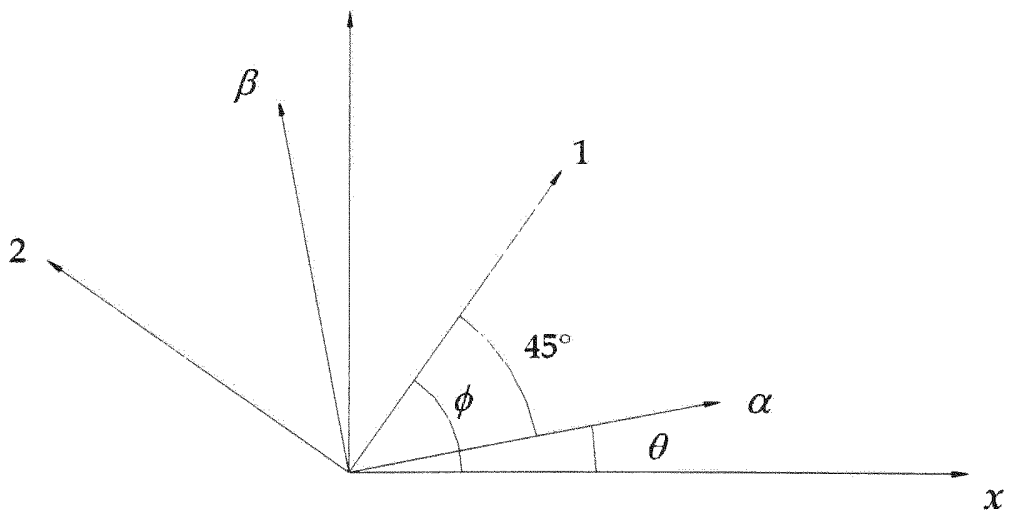

Fig. 2.1 Principal stress directions and the $\alpha$ and $\beta$ directions at a point in a plastically deforming region. 
Then from the equations for the principal directions, it follows that

$$
\tan 2 \phi=\frac{2 \tau_{x y}}{\sigma_{x}-\sigma_{y}}
$$

which gives two values of $\phi$ differing by $90^{\circ}$. The second principal direction is taken $90^{\circ}$ counterclockwise from the first. It should be noted that the intermediate principal stress, $\sigma_{2}$, has a direction which is normal to Fig. 2.1 in the direction of zero strain.

The maximum shear stresses have the values

$$
\tau_{\max }= \pm \frac{1}{2}\left(\sigma_{1}-\sigma_{3}\right)= \pm k
$$

The maximum shear stresses act on surfaces which make angles of $\pm \pi / 4$ with the principal directions. The directions of these surfaces on which the shear stress attains a maximum value $k$ are usually designated the $\alpha$ and $\beta$ directions. $\alpha$, called the first shear direction, is taken $45^{\circ}$ clockwise from the first principal direction, and $\beta$, the second shear direction, is $90^{\circ}$ counterclockwise from the first shear direction. 
Let $\theta$ be the angle which the first shear direction makes with the $x$ axis measured counterclockwise. Then,

$$
\begin{aligned}
& \theta=\phi-\pi / 4 \\
& \tan 2 \theta=-\frac{1}{\tan 2 \phi}
\end{aligned}
$$

and, from Eqs. (2.8) and (2.10)

$$
\tan 2 \theta=\frac{\sigma_{y}-\sigma_{x}}{2 \tau_{x y}}
$$

It follows that

$$
\begin{aligned}
& \cos 2 \theta=\frac{\tau_{x y}}{k} \\
& \sin 2 \theta=\frac{\sigma_{y}-\sigma_{x}}{2 k}
\end{aligned}
$$

At every point in the plastic field, the angle which the maximum shear direction makes with the $x$ axis is determined by equations (2.11) or (2.12). If curves are now drawn in the $x y$ plane such that at every point of each curve the tangent coincides with one of maximum shear directions, then two families of curves called shear lines, or slip lines, will be obtained. Since the maximum and 
minimum shear directions at a point are orthogonal to each other, the two families of slip lines will form an orthogonal set.

It should be carefully noted that along an $\alpha$ line $\alpha$ is varying and $\beta$ is constant, and along a $\beta$ line $\beta$ is varying and $\alpha$ is constant. $\alpha$ and $\beta$ are merely parameters or curvilinear coordinates used to designate the point under consideration, just as $x$ and $y$ designate the point.

The normal stresses acting on the maximum shear planes equals the average of the principal stresses. Thus the stresses acting normal and tangential to the $\alpha$ and $\beta$ lines are given by

$$
\begin{aligned}
& \sigma_{\alpha}=\sigma_{\beta}=\frac{1}{2}\left(\sigma_{1}+\sigma_{2}\right)=p \\
& \tau_{\alpha \beta}=\frac{1}{2}\left(\sigma_{1}-\sigma_{2}\right)=k
\end{aligned}
$$

Finally, $\sigma_{x}, \sigma_{y}$ and $\tau_{x y}$ can be expressed in terms of $p$ and $\theta$ as follows:

$$
\begin{aligned}
& \sigma_{x}=p-k \sin 2 \theta \\
& \sigma_{y}=p+k \sin 2 \theta \\
& \tau_{x y}=k \cos 2 \theta
\end{aligned}
$$




\subsection{Hencky Stress Equations}

The state of stress at a point can be expressed by equation (2.14) in terms of the independent quantities $p, k$, and $\theta$. The equilibrium equation can be written in terms of these quantities by substituting (2.14) into (2.4). Thus,

$$
\begin{aligned}
& \frac{\partial p}{\partial x}-2 k\left(\cos 2 \theta \frac{\partial \theta}{\partial x}+\sin 2 \theta \frac{\partial \theta}{\partial y}\right)=0 \\
& \frac{\partial p}{\partial y}+2 k\left(\cos 2 \theta \frac{\partial \theta}{\partial y}-\sin 2 \theta \frac{\partial \theta}{\partial x}\right)=0
\end{aligned}
$$

Equations (2.15) are the partial differential equations of equilibrium for the plane strain deformation of a rigid-perfectly plastic material and are hyperbolic. A rigorous solution to these equations can be obtained by the method of characteristics. The characteristic curves or characteristics of the hyperbolic, in this case, coincide with the slip lines.

The choice of $x$ and $y$ in equations (2.15) is arbitrary. If the $x$ and $y$ axes are chosen at a given point to coincide with the $\alpha$ and $\beta$ directions at this point, then $\theta=0$ and 


$$
\frac{\partial}{\partial x}=\frac{\partial}{\partial \alpha} \quad \frac{\partial}{\partial y}=\frac{\partial}{\partial \beta}
$$

equation (2.15) become

$$
\begin{aligned}
& \frac{\partial p}{\partial \alpha}-2 k \frac{\partial \theta}{\partial \alpha}=0 \\
& \frac{\partial p}{\partial \beta}+2 k \frac{\partial \theta}{\partial \beta}=0
\end{aligned}
$$

Since the point for which these equations are valid is arbitrary, it follows that equations (2.17) are applicable to all point along the slip lines. Integrating thus produces the following relationships applicable to the slip lines

$$
\begin{aligned}
& p-2 k \theta=C_{1} \quad \text { along the } \alpha \text { curve } \\
& p+2 k \theta=C_{2} \quad \text { along the } \beta \text { curve }
\end{aligned}
$$

where $C_{1}$ and $C_{2}$ are constants. These equations were first derived by Hencky in 1923. It is evident that if $p$ and $\theta$ are prescribed on the boundary, then it may be possible to proceed along constant $\alpha$ and $\beta$ lines to determine the value of the hydrostatic pressure everywhere in the slip line field. If the displacement or velocities are prescribed over part of the boundary, as is sometimes the case, 
then the Hencky stress equations are not sufficient to obtain a solution. It is then necessary to use the velocity equations.

\subsection{Geiringer Velocity Equations}

Consider an element in a plastically deforming region in the vicinity of certain point. Let its velocity at a given instant be $V$ and the components of this velocity along the $\alpha$ and $\beta$ slip lines be $v_{\alpha}$ and $v_{\beta}$, respectively.

Considering the velocity components $v_{\alpha}$ and $v_{\beta}$ along the slip lines in the $x$ and $y$ directions gives:

$$
\begin{aligned}
& v_{x}=v_{\alpha} \cos \theta-v_{\beta} \sin \theta \\
& v_{y}=v_{\alpha} \sin \theta+v_{\beta} \cos \theta
\end{aligned}
$$

If the element crosses a slip line it may be subjected to shear deformation in the shear direction. However, since it is assumed that only maximum shear stress can exist along shear lines and the normal stresses are everywhere equal to the hydrostatic pressure, no extension or contraction can occur along the slip lines although the element can distort in pure shear. Therefore, 


$$
\frac{\partial_{\alpha}}{\partial \alpha}=\frac{\partial_{\beta}}{\partial \beta}=0
$$

If $\theta=0$, the $x$ and $y$ axes will coincide with the $\alpha$ and $\beta$ directions. Then,

$$
\begin{aligned}
& \frac{\partial_{\alpha}}{\partial \alpha}=\frac{\partial v_{x}}{\partial x}=0 \\
& \frac{\partial_{\beta}}{\partial \beta}=\frac{\partial_{y}}{\partial y}=0
\end{aligned}
$$

Differentiating (2.19) and substituting (2.21) it follows:

$$
\begin{aligned}
& \frac{\partial_{\alpha}}{\partial \alpha}-v_{\beta} \frac{\partial \theta}{\partial \alpha}=0 \\
& \frac{\partial v_{\beta}}{\partial \beta}+v_{\alpha} \frac{\partial \theta}{\partial \beta}=0
\end{aligned}
$$

If $\beta$ is kept constant in the first equation and $\alpha$ in the second equation, then

$$
\begin{aligned}
& d v_{\alpha}-v_{\beta} d \theta=0 \quad \text { along an } \alpha \text { line } \\
& d v_{\beta}+v_{\alpha} d \theta=0 \quad \text { along an } \beta \text { line }
\end{aligned}
$$

These are the compatibility equations for velocities first derived by Geiringer in 1930. 
If the problem is statically determinate, the slip line field and the stresses can be defined from Eqs. (2.18) and the stress boundary conditions. The velocities can be determined from Eqs. (2.23) using the velocity boundary condition. However, if the problem is statically indeterminate when the stress boundary conditions are insufficient to obtain a unique slip line field then the Hencky stress equations must be solved simultaneously with the Geiringer velocity equations using both the stress boundary conditions and the velocity boundary condition. 


\section{CHAPTER 3}

\section{AN INTERFACE CRACK}

\subsection{Introduction}

In this work the mathematical model for the problem of the stresses inside the plastic region at the crack tip for an inhomogeneous material is based on the type of materials described in chapter 2. For this purpose, a composite material made of two different elastic-perfectly plastic materials with a crack is considered. This study is focused in the plastic region near the crack tip. The size of the plastic zone has the order of $K^{2} / \tau_{1}^{2}$ and $K^{2} / \tau_{2}^{2}$ in the first and second materials, where $K$ is the stress intensity factor computed for ideally elastic incompressible material pair with zero region size, and $\tau_{1}$ and $\tau_{2}$ are the yield stresses of the corresponding materials.

To estimate stresses at a crack tip inside the plastic region, it is convenient to use the model of an incompressible elastic-perfectly plastic material obeying the Huber-Mises yielding condition criterion. The second invariant of the stress deviator tensor in the material is the only material constant in this model. For plane strain, the Huber-Mises criterion coincides with the maximum shear stress or Tresca criterion. 
Following Cherepanov (1997) the solution for the stresses inside the plastic region in the case of an interface crack between two different elastic-perfectly plastic materials is presented in this chapter. The analysis of the solution, as well as the solution for the velocities, is given here for the first time and will be published elsewhere.

\subsection{Problem Statement}

Mathematically, the problem of stresses at the crack front is reduced to the following boundary value problem in plane strain:

$$
\begin{aligned}
& \frac{\partial \sigma_{x}}{\partial x}+\frac{\partial \tau_{x y}}{\partial y}=0 \\
& \frac{\partial \tau_{x y}}{\partial x}+\frac{\partial \sigma_{y}}{\partial y}=0 \\
& \left(\sigma_{y}-\sigma_{x}\right)^{2}+4 \tau_{x y}^{2}=\left\{\begin{array}{lll}
4 \tau_{1}^{2} & \text { when } & y>0 \\
4 \tau_{2}^{2} & \text { when } & y<0
\end{array}\right.
\end{aligned}
$$


Here, $x$ and $y$ are Cartesian rectangular coordinates, $\sigma_{x}, \tau_{x y}$ and $\sigma_{y}$ are stresses, and $\tau_{1}$ and $\tau_{2}$ are shear stress yield limits of the first and second solids correspondingly. From now on, in the yield criterion, Eq. (2.3), $k$ will take the value of $\tau_{1}$ or $\tau_{2}$ depending on the material 1 or material 2 respectively. The brackets $[. .$.$] denote the discontinuity of the quantity in the brackets along the$ bonded interface.

The non-linear equation system (3.1) to (3.3) belongs to the hyperbolic type with two families of characteristic lines issuing from a free boundary under 45 degrees to the tangent at every point. As was explained in chapter 2, the characteristics of the hyperbolic equations, in this case, coincide with the slip lines. From here, it follows that, in the rectangular equilateral triangles $O A B$ and OAF (see Fig. 3.1) having traction-free crack banks as the diagonal, the only stress field is:

$$
\sigma_{y}=\tau_{x y}=0, \quad \sigma_{x}=2 \tau_{1} \text { in } \triangle \mathrm{OAB}, \quad \sigma_{x}=2 \tau_{2} \text { in } \triangle \mathrm{OAF}
$$

The positive sign of $\sigma_{x}$ chosen in both triangles means aposteriori that the opening mode tensile crack is considered (this is correct for the model under study, but not for an elastic-brittle crack!). The crack tip, $O$, is the singular point of the boundary value problem. 


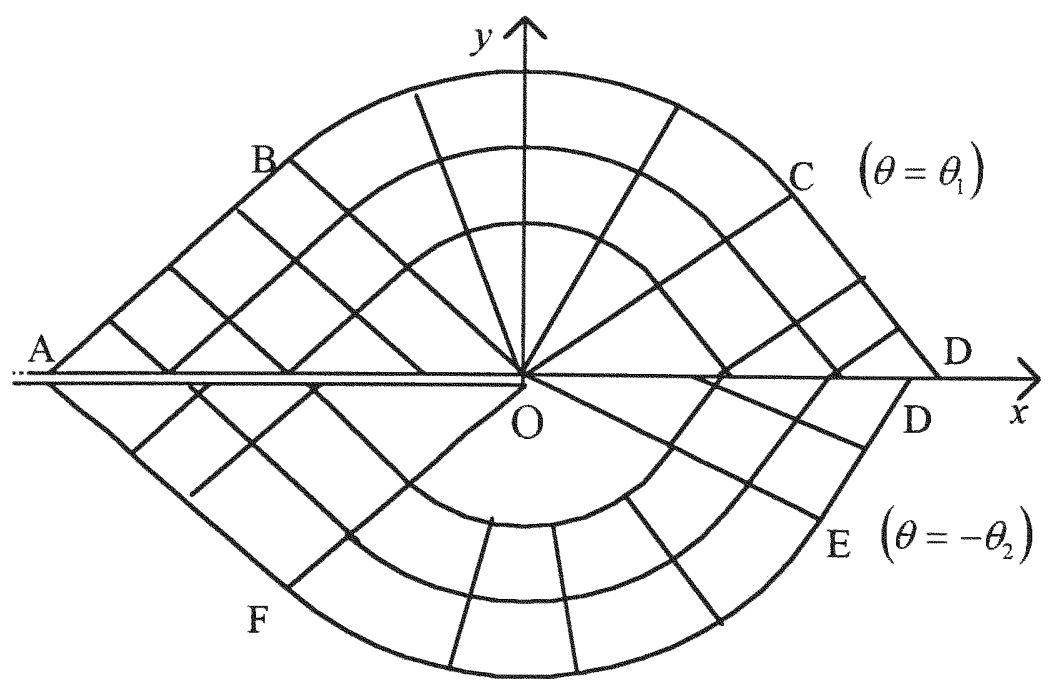

Fig. 3.1 The interface crack tip between two perfectly plastic materials: the geometry of characteristic lines in the crack tip neighborhood.

The solution has the following form:

a. In curvilinear triangles $\mathrm{OBC}$ and $\mathrm{OFE}$, the stresses $\sigma_{r}, \sigma_{\theta}$ and $\tau_{r \theta}$ are some functions of only $\theta$.

b. In rectangular triangles $\mathrm{OCD}$ and $\mathrm{ODE}$, the stresses are some constants.

Here, $r$ and $\theta$ are polar coordinates centered at $\mathrm{O}$, and $\sigma_{r}, \sigma_{\theta}$ and $\tau_{r \theta}$ are the corresponding stresses. 
The characteristic lines in $\triangle O B C$ and $\triangle O F E$ form a "fan" by radial and concentric lines (only in the first solution), and in $\triangle O C D$ and $\triangle O D E$ the characteristic lines form two rectangular grids of different orientation.

Equilibrium and yielding condition equations in polar coordinates are:

$$
\begin{aligned}
& \frac{d \tau_{r \theta}}{d \theta}+\sigma_{r}-\sigma_{\theta}=0 \\
& \frac{d \sigma_{\theta}}{d \theta}+2 \tau_{r \theta}=0 \\
& \left(\sigma_{r}-\sigma_{\theta}\right)^{2}+4 \tau_{r \theta}^{2}=\left\{\begin{array}{lll}
4 \tau_{1}^{2} & \text { when } & y>0 \\
4 \tau_{2}^{2} & \text { when } & y<0
\end{array}\right.
\end{aligned}
$$

Introduce the new function, $f(\theta)$ :

$$
\begin{aligned}
& \sigma_{r}-\sigma_{\theta}=2 \tau_{i} \sin f(\theta) \\
& \tau_{r \theta}=\tau_{i} \cos f(\theta)
\end{aligned} \quad(i=1,2)
$$

so that, Eq. (3.9) is met in the upper triangle for $i=1$, and in the lower triangle for $i=2$. Substituting Eq. (3.10) into Eqs. (3.7) and (3.8) provides 


$$
\begin{aligned}
& \left(2-\frac{d f}{d \theta}\right) \sin f(\theta)=0 \\
& \frac{d \sigma_{\theta}}{d \theta}=-2 \tau_{i} \cos f(\theta) .
\end{aligned}
$$

As a result, we have two possible solutions for $i=1$ :

1. $\sin f(\theta)=0, \quad \cos f(\theta)= \pm 1$

or

$$
\text { 2. } f=2 \theta+C_{1}, \quad \sigma_{\theta}=-\tau_{1} \sin \left(2 \theta+C_{1}\right)+C_{2} .
$$

The corresponding stress field is:

1. $\sigma_{r}=\sigma_{\theta}=\mp 2 \tau_{1} \theta+C_{3}, \quad \tau_{r \theta}= \pm \tau_{1}$

or

$$
\text { 2. } \begin{aligned}
\sigma_{r} & =\tau_{1} \sin \left(2 \theta+C_{1}\right)+C_{2}, \\
\sigma_{\theta} & =-\tau_{1} \sin \left(2 \theta+C_{1}\right)+C_{2}, \\
\tau_{r \theta} & =\tau_{1} \cos \left(2 \theta+C_{1}\right)
\end{aligned}
$$

Here, $C_{1}, C_{2}$ and $C_{3}$ are arbitrary constants. In Eq. (3.15), either the upper or lower sign should be taken. 
By replacing $\tau_{1}$ with $\tau_{2}$, the solutions for $\triangle \mathrm{OFE}$ can be found. Consequently, for $i=2$ it follows in $\triangle \mathrm{OFE}$

1. $\sigma_{r}=\sigma_{\theta}=\mp 2 \tau_{2} \theta+C_{3}, \quad \tau_{r \theta}= \pm \tau_{2}$

or

$$
\text { 2. } \begin{aligned}
\sigma_{r} & =\tau_{2} \sin \left(2 \theta+C_{1}\right)+C_{2}, \\
\sigma_{\theta} & =-\tau_{2} \sin \left(2 \theta+C_{1}\right)+C_{2}, \\
\tau_{r \theta} & =\tau_{2} \cos \left(2 \theta+C_{1}\right)
\end{aligned}
$$

Equations (3.15) and (3.17) correspond to a continuous solution and equations (3.16) and (3.18) correspond to a discontinuous solution.

\subsection{Continuous Solution}

According to Eq. (3.6), it follows:

$$
\sigma_{\theta}=\tau_{1}, \quad \tau_{r \theta}=\tau_{1} \quad \text { along } \quad \theta=\frac{3 \pi}{4}
$$

The stresses $\sigma_{\theta}$ and $\tau_{r \theta}$ should be continuous along OB. Matching the solutions given by Eqs. (3.15) with this condition it can be found in $\triangle O B C$ : 


$$
\sigma_{r}=\sigma_{\theta}=-2 \tau_{1} \theta+\tau_{1}\left(1+\frac{3 \pi}{2}\right), \quad \tau_{r \theta}=\tau_{1}
$$

Similarly, we can find in $\triangle \mathrm{OFE}$ :

$$
\sigma_{r}=\sigma_{\theta}=+2 \tau_{2} \theta+\tau_{2}\left(1+\frac{3 \pi}{2}\right), \quad \tau_{r \theta}=-\tau_{2}
$$

In the triangles OCD and ODE, the $\sigma_{x}$ are different and the $\sigma_{y}$ and $\tau_{x y}$ are the same due to boundary condition (3.5). Using Eq. (3.3) it follows:

$$
\sigma_{x}= \begin{cases}\sigma_{y}-2 \sqrt{\tau_{1}^{2}-\tau_{x y}^{2}} & (\Delta \mathrm{OCD}) \\ \sigma_{y}-2 \sqrt{\tau_{2}^{2}-\tau_{x y}^{2}} & (\Delta \mathrm{ODE})\end{cases}
$$

Assumed here is that $\sigma_{y}>\sigma_{x}$ in these triangles which is physically evident for opening mode tensile cracks.

The stresses, $\sigma_{\theta}$ and $\tau_{r \theta}$, should be continuous along $O C$ and $O E$. Designate: $\theta=\theta_{1}$ along $\mathrm{OC}$ and $\theta=-\theta_{2}$ along $\mathrm{OE}$. From here, it follows that: 


$$
\begin{aligned}
& -2 \tau_{1} \theta_{1}+\tau_{1}\left(1+\frac{3 \pi}{2}\right)=\left(\sigma_{y}-2 \sqrt{\tau_{1}^{2}-\tau_{x y}^{2}}\right) \sin ^{2} \theta_{1}+ \\
& +\sigma_{y} \cos ^{2} \theta_{1}-2 \tau_{x y} \sin \theta_{1} \cos \theta_{1} ; \\
& \tau_{1}=\sqrt{\tau_{1}^{2}-\tau_{x y}^{2}} \sin 2 \theta_{1}+\tau_{x y} \cos 2 \theta_{1} ; \\
& -2 \tau_{2} \theta_{2}+\tau_{2}\left(1+\frac{3 \pi}{2}\right)=\left(\sigma_{y}-2 \sqrt{\tau_{2}^{2}-\tau_{x y}^{2}}\right) \sin ^{2} \theta_{2}+ \\
& +\sigma_{y} \cos ^{2} \theta_{2}+2 \tau_{x y} \sin \theta_{2} \cos \theta_{2} ; \\
& -\tau_{2}=-\sqrt{\tau_{2}^{2}-\tau_{x y}^{2}} \sin 2 \theta_{2}+\tau_{x y} \cos 2 \theta_{2} .
\end{aligned}
$$

There are four equations for determining $\theta_{1}, \theta_{2}, \sigma_{y}$, and $\tau_{x y}$. Below it will be shown that Eqs. (3.23) have the physical meaning only when $1 \geq \tau_{1} / \tau_{2} \geq 0.71$.

Excluding $\sigma_{y}$ and $\tau_{x y}$ in the equation system (3.23), the following equations for the continuous solution can be derived:

$$
\begin{aligned}
& \lambda \cos 2 \theta_{1}=-\cos 2 \theta_{2} \\
& 2\left(\theta_{2}-\lambda \theta_{1}\right)-(1-\lambda)\left(1+\frac{3 \pi}{2}\right)=\sin 2 \theta_{2}-\lambda \sin 2 \theta_{1}
\end{aligned}
$$

where

$$
\lambda=\frac{\tau_{1}}{\tau_{2}}
$$


The stresses, $\sigma_{y}$ and $\tau_{x y}$, in the triangles OCD and ODE are equal to:

$$
\begin{aligned}
& \sigma_{y}=\tau \sin 2 \theta+\tau\left(1+\frac{3 \pi}{2}\right)-2 \tau \theta, \quad \text { when } \theta_{1}>\theta>-\theta_{2} \\
& \tau_{x y}=\tau \cos 2 \theta, \\
& \text { and } \tau=\tau_{1} \text { when } 0<\theta<\theta_{1} \text { and } \tau=-\tau_{2} \text { when } 0>\theta>-\theta_{2} \text {. }
\end{aligned}
$$

When $\tau_{1}=\tau_{2}$, from Eqs. (3.24) and (3.25) it follows that $\theta_{1}=\theta_{2}=\pi / 4$, and

$$
\begin{aligned}
& \sigma_{y}=\tau_{1}(2+\pi), \\
& \sigma_{x}=\pi \tau_{1}, \\
& \tau_{x y}=0,
\end{aligned}
$$

This solution was obtained by Prandtl for the problem of a rigid smooth punch which as was shown by Hill and Sokolovsky, is mathematically identical to the crack problem (in homogeneous case). This solution is known as the Prandtl solution. A modified form of this solution was also studied by Hill and Sokolovsky.

The numerical solution of the system (3.24) provides $\theta_{1}$ and $\theta_{2}$ in terms of $\lambda$. This solution is shown in Table 3.1 and Figs. 3.2 and 3.3. Figures 3.4, 3.5 and 3.6 
show the graphs $\sigma_{y} / \tau_{1}$ vs. $\lambda, \tau_{x y} / \tau_{1}$ vs. $\lambda$ and $\sigma_{x} / \tau_{1}$ vs. $\lambda$ respectively, based on Eqs. (3.22) and (3.25).

Table 3.1. Numerical solution of the equation system (3.24), when $1 \geq \lambda \geq 0.71$.

\begin{tabular}{|c|c|c|c|c|c|c|c|c|c|c|}
\hline$\lambda=\frac{\tau_{1}}{\tau_{2}}$ & 1 & 0.9366 & 0.8818 & 0.8351 & 0.7962 & 0.7647 & 0.7405 & 0.7234 & 0.7133 & 0.71 \\
\hline$\theta_{1}$ & 45 & 40 & 35 & 30 & 25 & 20 & 15 & 10 & 5 & 0 \\
\hline$\theta_{2}$ & 45 & 49.68 & 53.77 & 57.34 & 60.39 & 62.93 & 64.94 & 66.41 & 67.31 & 67.61 \\
\hline
\end{tabular}

From the solution of the equation system (3.24) (Table 3.1), it is observed that the solution exists only for $0.71 \leq \lambda \leq 1$. The upper limiting case is the homogeneous solution and the lower limiting case is for $\lambda=0.71, \theta_{1}=0$, and $\theta_{2}=67.61^{\circ}$. 


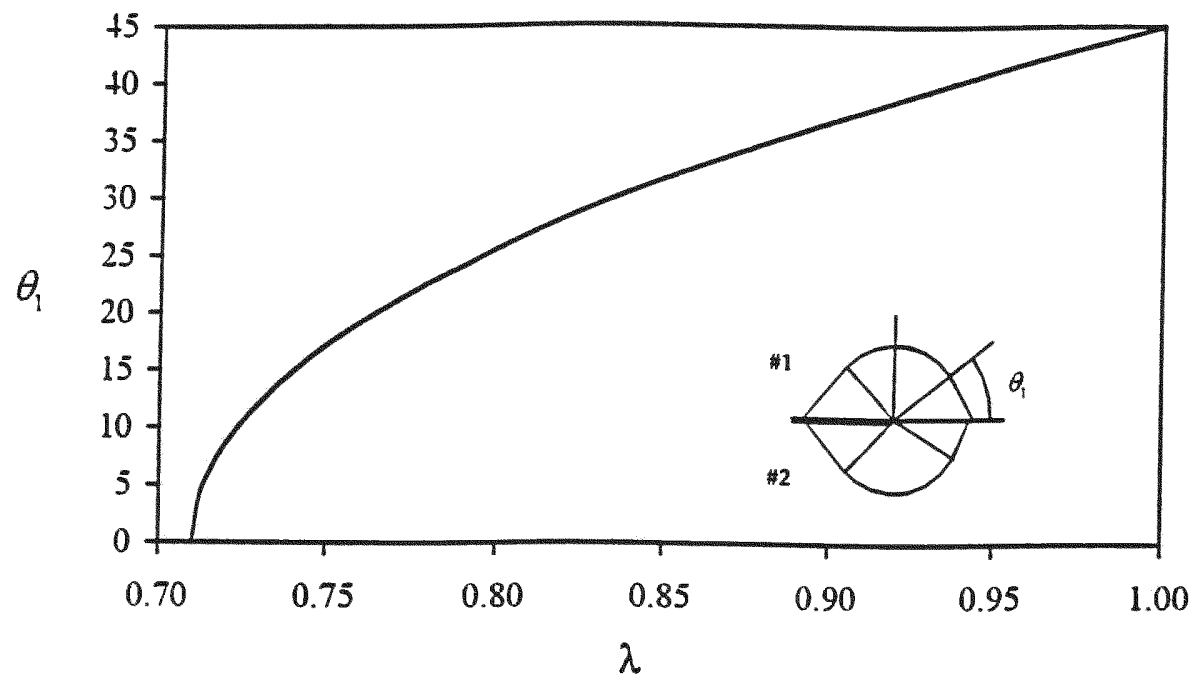

Fig 3.2 Graph $\theta_{1}$ vs. $\lambda=\tau_{1} / \tau_{2}$ for the continuous solution of the inhomogeneous problem, Eq. (3.24).

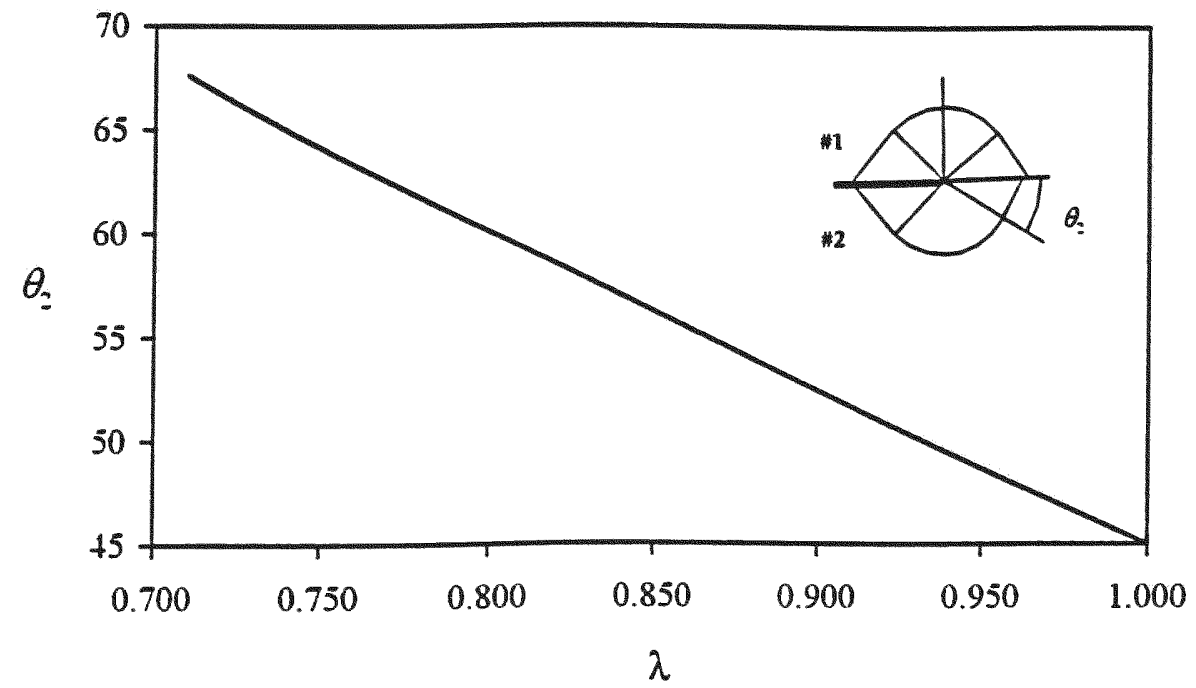

Fig 3.3 Graph $\theta_{2}$ vs. $\lambda=\tau_{1} / \tau_{2}$ where $\theta_{2}=\frac{1}{2} \cos ^{-1}\left[-\lambda \cos 2 \theta_{1}\right]$ for the continuous solution of the inhomogeneous problem, Eq. (3.24). 


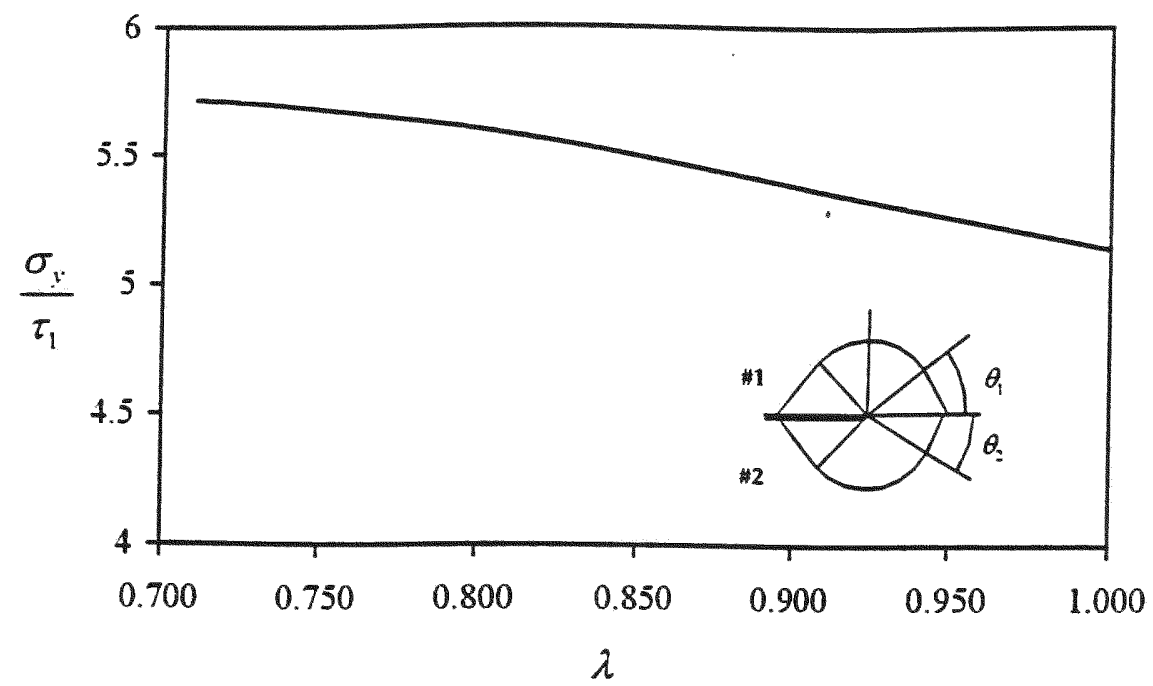

Fig 3.4 Graph $\frac{\sigma_{y}}{\tau_{1}}$ vs. $\lambda=\frac{\tau_{1}}{\tau_{2}}$ for $0.71 \leq \lambda \leq 1$.

The $\sigma_{y}$ is the corresponding stress for $\theta_{1}>\theta>-\theta_{2}$.

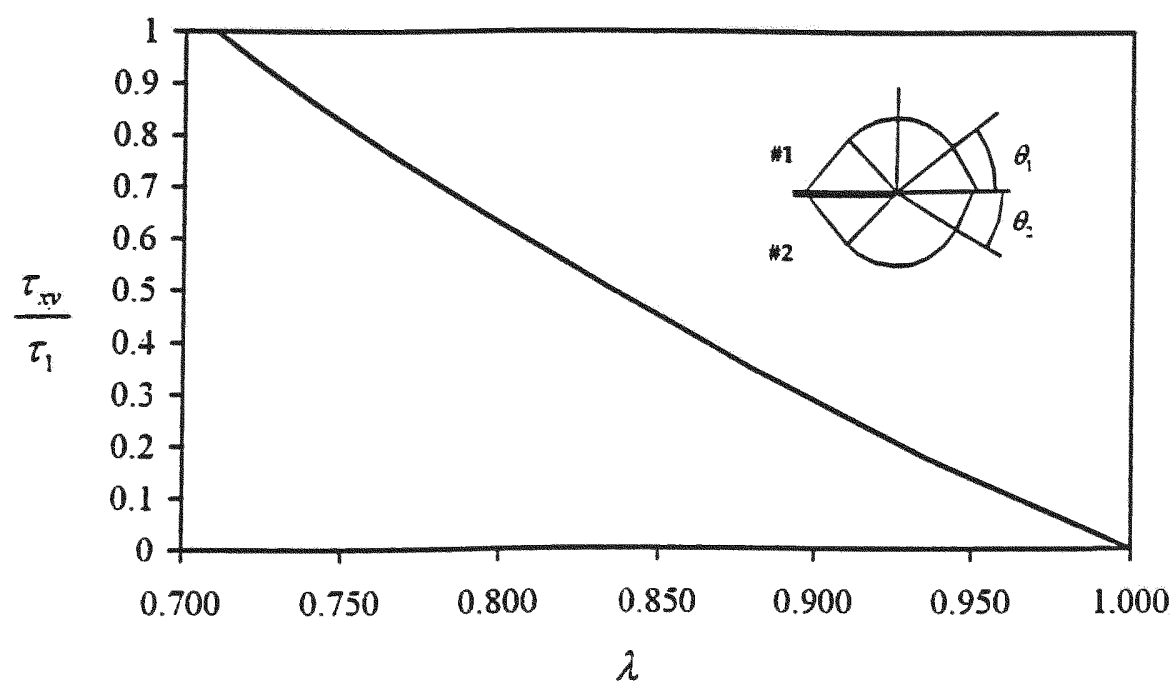

Fig $3.5 \mathrm{Graph} \frac{\tau_{x v}}{\tau_{1}}$ vs. $\lambda=\frac{\tau_{1}}{\tau_{2}}$ for $0.71 \leq \lambda \leq 1$.

The $\tau_{x y}$ is the corresponding stress for $\theta_{1}>\theta>-\theta_{2}$. 


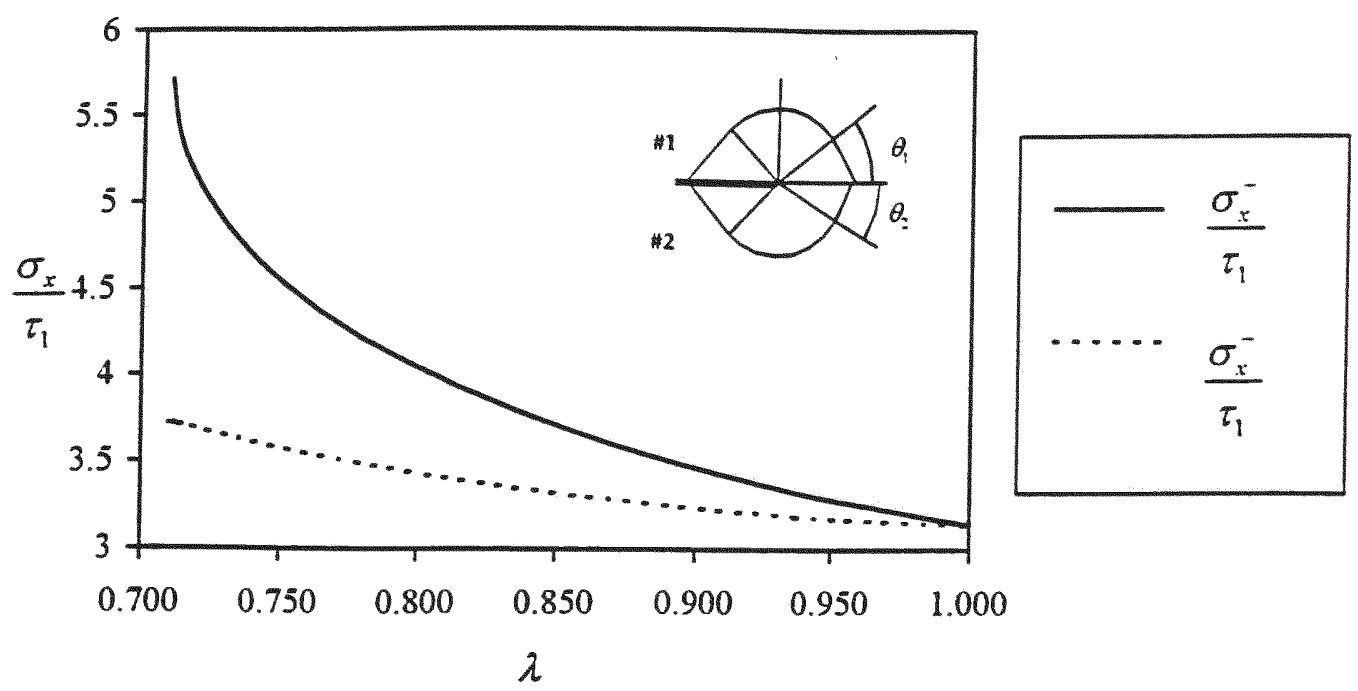

Fig 3.6 Graph $\frac{\sigma_{x}^{-}}{\tau_{1}}$ and $\frac{\sigma_{x}^{-}}{\tau_{1}}$ vs. $\lambda=\frac{\tau_{1}}{\tau_{2}}$ for $0.71 \leq \lambda \leq 1$.

The $\sigma_{x}^{*}$ is the corresponding stress for $\theta_{1}>\theta>0$, and

The $\sigma_{x}^{-}$is the corresponding stress for $0>\theta>-\theta_{2}$.

\subsection{Discontinuous Solution}

Similar to the analysis of the continuos solution, according to Eq. (3.6) it follows

$$
\sigma_{\theta}=\tau_{1}, \quad \tau_{r \theta}=\tau_{1} \quad \text { along } \quad \theta=\frac{3 \pi}{4}
$$

The stresses $\sigma_{\theta}$ and $\tau_{r \theta}$ should be continuous along $\mathrm{OB}$. Matching the solutions given by Eqs. (3.16) with this condition we can find in $\triangle O B C$ : 


$$
\begin{aligned}
& \sigma_{r}=\tau_{1} \cos 2 \theta+\tau_{1}, \\
& \sigma_{\theta}=-\tau_{1} \cos 2 \theta+\tau_{1}, \\
& \tau_{r \theta}=-\tau_{1} \sin 2 \theta . \quad(\Delta \mathrm{OBC})
\end{aligned}
$$

Similarly, we can find in $\triangle \mathrm{OFE}$ :

$$
\begin{aligned}
\sigma_{r} & =\tau_{2}(1+\cos 2 \theta), \\
\sigma_{\theta} & =\tau_{2}(1-\cos 2 \theta), \\
\tau_{r \theta} & =-\tau_{2} \sin 2 \theta . \quad(\Delta \mathrm{OFE})
\end{aligned}
$$

In the triangles OCD and ODE, the $\sigma_{x}$ are different and the $\sigma_{y}$ and $\tau_{x y}$ are the same due to boundary condition (3.5). Using Eq. (3.3) it follows:

$$
\sigma_{x}= \begin{cases}\sigma_{y}-2 \sqrt{\tau_{1}^{2}-\tau_{x y}^{2}} & (\Delta \mathrm{OCD}) \\ \sigma_{y}-2 \sqrt{\tau_{2}^{2}-\tau_{x y}^{2}} & (\Delta \mathrm{ODE})\end{cases}
$$

Assumed here is that $\sigma_{y}>\sigma_{x}$ in these triangles which is physically evident for opening mode tensile cracks. 
The stresses, $\sigma_{\theta}$ and $\tau_{r \theta}$, should be continuous along $\mathrm{OC}$ and $\mathrm{OE}$. Designate: $\theta=\theta_{1}$ along $\mathrm{OC}$ and $\theta=-\theta_{2}$ along $\mathrm{OE}$. From here, it follows that:

$$
\begin{aligned}
& \tau_{1}\left(1-\cos 2 \theta_{1}\right)=\left(\sigma_{y}-2 \sqrt{\tau_{1}^{2}-\tau_{x y}^{2}}\right) \sin ^{2} \theta_{1}+ \\
& +\sigma_{y} \cos ^{2} \theta_{1}-2 \tau_{x y} \sin \theta_{1} \cos \theta_{1} ; \\
& -\tau_{1} \sin 2 \theta_{1}=\sqrt{\tau_{1}^{2}-\tau_{x y}^{2}} \sin 2 \theta_{1}+\tau_{x y} \cos 2 \theta_{1} ; \\
& \tau_{2}\left(1-\cos 2 \theta_{2}\right)=\left(\sigma_{y}-2 \sqrt{\tau_{2}^{2}-\tau_{x y}^{2}}\right) \sin ^{2} \theta_{2}+ \\
& +\sigma_{y} \cos ^{2} \theta_{2}+2 \tau_{x y} \sin \theta_{2} \cos \theta_{2} ; \\
& \tau_{2} \sin 2 \theta_{2}=-\sqrt{\tau_{2}^{2}-\tau_{x y}^{2}} \sin 2 \theta_{2}+\tau_{x y} \cos 2 \theta_{2} .
\end{aligned}
$$

There are four equations for determining $\theta_{1}, \theta_{2}, \sigma_{y}$, and $\tau_{x y}$.

Excluding $\sigma_{y}$ and $\tau_{x y}$ in the equation system (3.31), the following equations can be derived:

$$
\begin{aligned}
& \lambda \sin 4 \theta_{1}=-\sin 4 \theta_{2} \\
& \lambda\left(1-\cos 2 \theta_{1}+2 \cos 4 \theta_{1} \sin ^{2} \theta_{1}-\sin 4 \theta_{1} \sin 2 \theta_{1}\right)= \\
& =\left(1-\cos 2 \theta_{2}+2 \cos 4 \theta_{2} \sin ^{2} \theta_{2}-\sin 4 \theta_{2} \sin 2 \theta_{2}\right) \quad\left(\lambda=\tau_{1} / \tau_{2}\right) .
\end{aligned}
$$

The stresses, $\sigma_{y}$ and $\tau_{x y}$, in the triangles OCD and ODE (Fig. 2) are equal to: 


$$
\begin{aligned}
& \sigma_{y}=\tau\left(1-\cos 2 \theta+2 \cos 4 \theta \sin ^{2} \theta-\sin 4 \theta \sin 2 \theta\right), \\
& \tau_{x y}=-\tau \sin 4 \theta, \text { when } \theta_{1}>\theta>-\theta_{2},
\end{aligned}
$$

where $\tau=\tau_{1}$ when $0<\theta<\theta_{1}$ and $\tau=\tau_{2}$ when $0>\theta>-\theta_{2}$.

The numerical analysis has shown that the equation system (3.32) has only the following solutions:

Case 1.

$$
\theta_{1}=\theta_{2}=0 \quad \text { for any } \lambda
$$

Case 2.

$$
\theta_{1}=\theta_{2}=\frac{\pi}{4} \quad \text { for any } \lambda
$$

Case 3.

$$
\theta_{1}=\theta_{2}=\frac{\pi}{2} \quad \text { when } \lambda=1 \quad \text { (homogeneous case) }
$$

For the first and second case, from Eq. (3.31) it follows, that in these cases:

$$
\sigma_{y}=\tau_{x y}=0, \quad \text { and } \quad \sigma_{x}=-2 \tau \quad \text { (in } \triangle \mathrm{OCD} \text { and } \triangle \mathrm{ODE} \text { of Fig. 2) }
$$

where $\tau=\tau_{1}$ when $0<\theta<\theta_{1}$ and $\tau=\tau_{2}$ when $0>\theta>-\theta_{2}$

which has no physical meaning. 
For a homogeneous material (Case 3), from equations (3.30), (3.33) and (3.36) it follows that in this case:

$$
\begin{aligned}
& \sigma_{y}=4 \tau_{1}, \\
& \sigma_{x}=2 \tau_{1}, \quad \text { when } \quad\left(\lambda=\frac{\tau_{1}}{\tau_{2}}=1\right) \\
& \tau_{x y}=0,
\end{aligned}
$$

which may have a physical meaning.

In the particular case when $\lambda=0, \theta_{2}=0, \pi / 4$ or $\pi / 2$ satisfies Eqs. (3.32) for any $\theta_{1}$, so that in this case from Eqs. (3.30), and (3.33) it follows:

$$
\begin{aligned}
& \sigma_{y}=4 \tau_{2}, \\
& \sigma_{x}=2 \tau_{2}, \quad \text { when } \theta_{2}=\frac{\pi}{2}, \quad\left(\lambda=0 \text { for any } \theta_{1}\right) \\
& \tau_{x y}=0,
\end{aligned}
$$

This solution corresponds to the punch problem when the stresses in the wedge ODE are given by Eq. (3.39). 
So, only in the cases of $\lambda=0$ or $\lambda=1$ the equation system (3.32) has the physically meaningful solutions.

The second solution at $\lambda=1$ is the discontinuous solution of the classic theory of plasticity unnoticed by Prandtl, Hill, and Sokolovsky. The structure of characteristic lines in this solution is shown in Fig. 3.7b while Fig. 3.7a gives the characteristic line structure in the classic Prandtl's solution.

\subsection{Velocity Distribution}

The characteristic lines of the Reuss equations for velocity coincide with those indicated above for stresses. Therefore, the velocity field is also formed by a combination of constant velocity fields and "fan" fields when:

$$
v_{r}=0 \text { and } v_{\theta}=V_{0} \text { in "fan", }
$$

where $v_{r}$ and $v_{\theta}$ are the radial and tangential velocity components respectively ( $v_{r}=0$ because of a rigid radial constraint and $V_{0}$ is a constant specified by boundary conditions). 
The results of simple calculations are given below:

1. The continuous solution:

$$
\begin{aligned}
& v_{x}=\frac{\sqrt{2}}{2} V_{0}, \quad v_{y}=\frac{\sqrt{2}}{2} V_{0} \quad \text { (in } \quad \triangle \mathrm{OAB}, \text { Fig.3.1) } \\
& v_{x}=V_{0} \sin \theta, \quad v_{y}=-V_{0} \cos \theta \quad \text { (in } \Delta \mathrm{OBC}, \text { Fig.3.1) } \\
& v_{x}=V_{0} \sin \theta_{1}, \quad v_{y}=-V_{0} \cos \theta_{1} \quad \text { (in } \Delta \mathrm{OCD}, \text { Fig. 3.1) }
\end{aligned}
$$

Along the interface the normal velocity is continuous. Therefore,

$$
v_{x}=-V_{0} \cos \theta_{1} \tan \theta_{2}, \quad v_{y}=-V_{0} \cos \theta_{1} \quad \text { (in } \triangle \mathrm{ODE}, \text { Fig.3.1) }
$$

$$
v_{x}=-V_{0} \frac{\cos \theta_{1}}{\cos \theta_{2}} \sin \theta, \quad v_{y}=-V_{0} \frac{\cos \theta_{1}}{\cos \theta_{2}} \cos \theta \quad \text { (in } \triangle \mathrm{OEF}, \text { Fig. 3.1) }
$$

$$
v_{x}=-\frac{\sqrt{2}}{2} V_{0} \frac{\cos \theta_{1}}{\cos \theta_{2}}, \quad v_{y}=\frac{\sqrt{2}}{2} V_{0} \frac{\cos \theta_{1}}{\cos \theta_{2}} \quad \text { (in } \triangle \mathrm{OAF}, \text { Fig.3.1) }
$$


2. The discontinuous solution. $\left(\lambda=1\right.$, that is, $\left.\tau_{1}=\tau_{2}\right)$ :

$v_{x}=\frac{\sqrt{2}}{2} V_{0}, \quad v_{y}=\frac{\sqrt{2}}{2} V_{0} \quad$ in $\triangle \mathrm{OAB}$, and $\mathrm{OBC}$, Fig.3.7b)

$v_{x}=\frac{\sqrt{2}}{2} V_{0}, \quad v_{y}=-\frac{\sqrt{2}}{2} V_{0} \quad$ (in $\triangle \mathrm{OCD}$, Fig. $3.7 \mathrm{~b}$ )

$v_{x}=-\frac{\sqrt{2}}{2} V_{0}, \quad v_{y}=-\frac{\sqrt{2}}{2} V_{0} \quad$ (in $\triangle \mathrm{ODE}$, Fig. $3.7 \mathrm{~b}$ )

$v_{x}=-\frac{\sqrt{2}}{2} V_{0}, \quad v_{y}=\frac{\sqrt{2}}{2} V_{0}$ (in $\triangle \mathrm{OEF}$, and OFA, Fig.3.7b) (3.50)

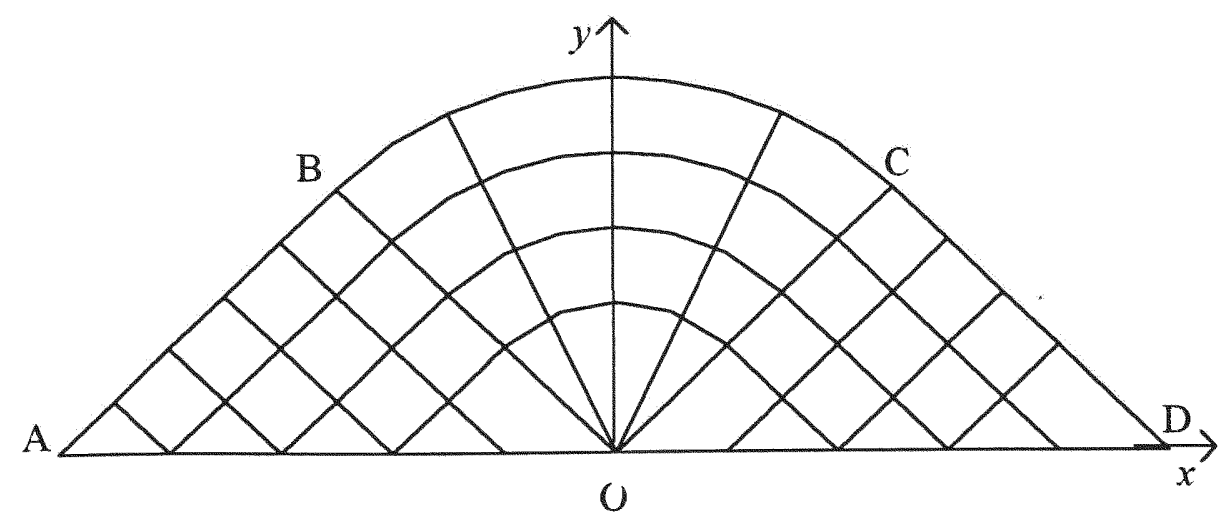

Fig 3.7a. Characteristic lines for the first solution of the plasticity theory.

$$
\sigma_{y}=\tau_{1}(2+\pi), \sigma_{x}=\pi \tau_{1}, \quad \tau_{x y}=0 \text { when }|\theta|<\frac{\pi}{4} .
$$




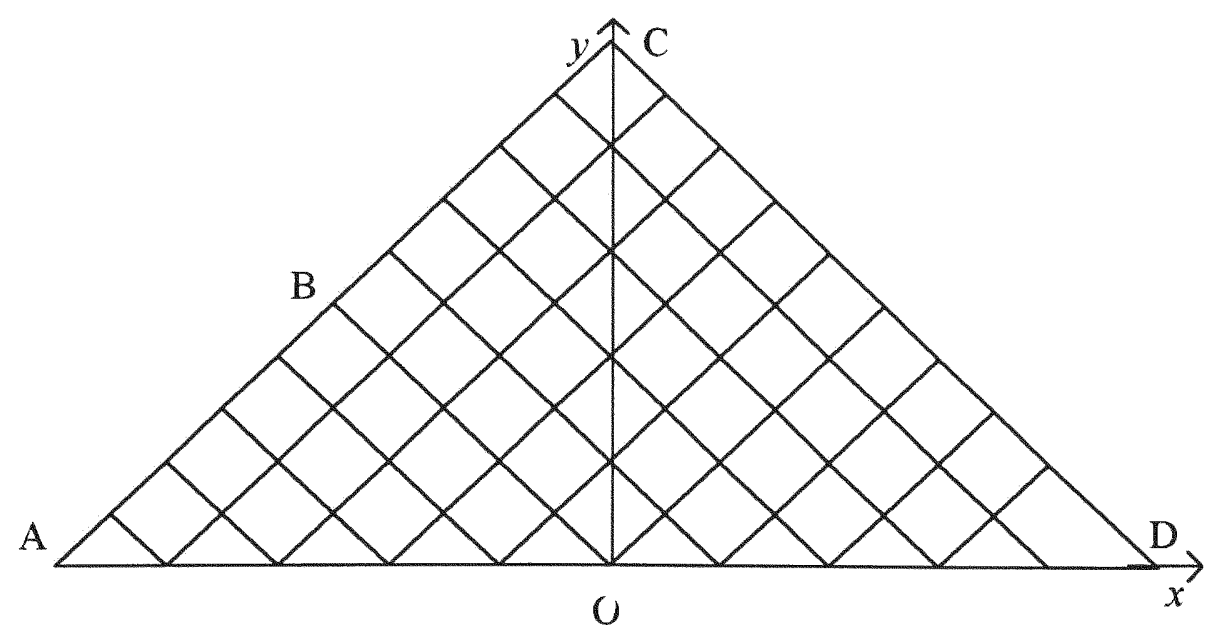

Fig 3.7b. Characteristic lines for the second solution of the plasticity theory.

$$
\sigma_{y}=4 \tau_{1}, \quad \sigma_{x}=2 \tau_{1}, \tau_{x y}=0 \text { when } \theta_{1}=\theta_{2}=\frac{\pi}{2}
$$




\section{CHAPTER 4}

\section{A CRACK IMPINGING THE INTERFACE AT A RIGTH ANGLE}

\subsection{Introduction}

In the previous chapter, the model of an incompressible elastic-perfectly plastic material obeying the Huber-Mises yielding criterion was considered and the condition of plane strain was assumed to estimate the stresses inside the plastic region using the theory of shear lines or slip lines. In chapter 3 the case of an interface crack between two different elastic-perfectly plastic solids was studied.

This chapter is devoted to the study of the stress state inside the plastic region at the crack tip when a crack impinges the interface of two different elasticperfectly plastic materials under the right angle (see Fig. 4.1 below). Here the same type of materials and assumptions used in chapter 3 are considered. The main difference with respect to the previous chapter is the boundary condition along the interface. 


\subsection{Problem Statement}

The problem of stresses at the crack front is reduced to the following boundary value problem in plane strain:

$$
\frac{\partial \sigma_{x}}{\partial x}+\frac{\partial \tau_{x y}}{\partial y}=0 \quad(|x|<\infty,|y|<\infty)
$$

$$
\begin{aligned}
& \frac{\partial \tau_{y x}}{\partial x}+\frac{\partial \sigma_{y}}{\partial y}=0 \quad(|x|<\infty,|y|<\infty), \\
& \left(\sigma_{y}-\sigma_{x}\right)^{2}+4 \tau_{x y}^{2}=\left\{\begin{array}{ll}
4 \tau_{1}^{2} & \text { when } x<0 \\
4 \tau_{2}^{2} & \text { when } x>0
\end{array},\right.
\end{aligned}
$$

$$
\sigma_{y}=\tau_{x y}=0 \quad \text { when } \quad y=0, x<0
$$

$$
\left[\sigma_{\theta}\right]=\left[\tau_{r \theta}\right]=0 \text { when } x=0 \text { and } \tau_{x y}=0 \text { when } y=0, x>0
$$

Here, the stress component $\sigma_{\theta}$ and the shear stress $\tau_{r \theta}$ should be continuous along the interface (line OC in Fig. 4.1). 
Again, the non-linear equation system (4.1) to (4.3) belongs to the hyperbolic type with two families of characteristic lines issuing from a free boundary under 45 degrees to the tangent at every point. From here, it follows that, in the rectangular equilateral triangle $\mathrm{OAB}$ (see Fig. 4.1) having traction-free crack banks as the diagonal, the only stress field is:

$$
\sigma_{y}=\tau_{x y}=0, \quad \sigma_{x}=2 \tau_{1} \quad \text { in } \quad \triangle \mathrm{OAB}
$$

The positive sign of $\sigma_{x}$ chosen in the triangle means aposteriori that the opening mode tensile crack is considered (this is correct for the model under study, but not for an elastic-brittle crack!). The crack tip, $O$, is the singular point of the boundary value problem. 


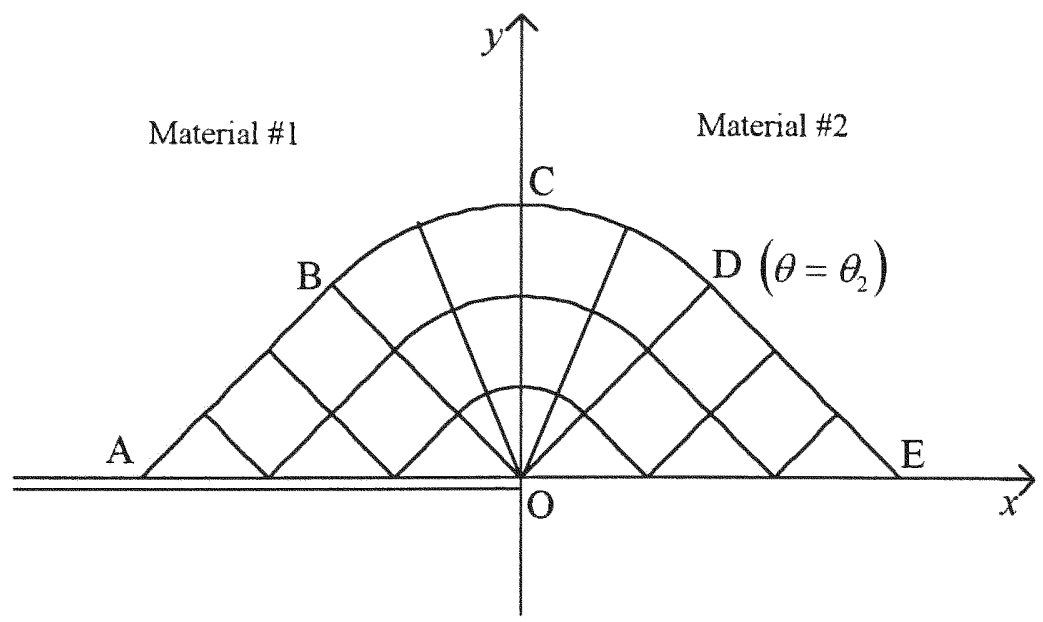

Fig. 4.1 The crack impinging the interface of two elastic-perfectly plastic materials bonded along $x=0$.

The solution has the following form:

a. In curvilinear triangles $\mathrm{OBC}$ and $\mathrm{OCD}$, the stresses $\sigma_{r}, \sigma_{\theta}$ and $\tau_{r \theta}$ are some functions of only $\theta$.

b. In rectangular triangle ODE, the stresses are some constants.

Here, $r$ and $\theta$ are polar coordinates centered at $\mathrm{O}$, and $\sigma_{r}, \sigma_{\theta}$ and $\tau_{r \theta}$ are the corresponding stresses.

Equilibrium and yielding condition equations in polar coordinates are: 


$$
\frac{d \tau_{r \theta}}{d \theta}+\sigma_{r}-\sigma_{\theta}=0
$$

$$
\frac{d \sigma_{\theta}}{d \theta}+2 \tau_{r \theta}=0
$$

$$
\left(\sigma_{r}-\sigma_{\theta}\right)^{2}+4 \tau_{r \theta}^{2}=\left\{\begin{array}{lll}
4 \tau_{1}^{2} & \text { when } & x<0 \\
4 \tau_{2}^{2} & \text { when } & x>0
\end{array}\right.
$$

Introduce the new function $f(\theta)$ :

$$
\begin{aligned}
& \sigma_{r}-\sigma_{\theta}=2 \tau_{1} \sin f(\theta) \\
& \tau_{r \theta}=\tau_{1} \cos f(\theta)
\end{aligned}
$$

so that, equation (4.9) is met in the region $\mathrm{OAB}(x<0)$. Substituting $(4.10)$ into (4.7) and (4.8) provides

$$
\begin{aligned}
& \left(2-\frac{d f}{d \theta}\right) \sin f(\theta)=0 \\
& \frac{d \sigma_{\theta}}{d \theta}=-2 \tau_{1} \cos f(\theta) .
\end{aligned}
$$


As a result, we have two possible solutions:

1. $\sin f(\theta)=0, \quad \cos f(\theta)= \pm 1$

or

$$
\text { 2. } f=2 \theta+C_{1}, \quad \sigma_{\theta}=-\tau_{1} \sin \left(2 \theta+C_{1}\right)+C_{2} \text {. }
$$

The corresponding stress field is:

$$
\text { 1. } \sigma_{r}=\sigma_{\theta}=\mp 2 \tau_{1} \theta+C_{3}, \quad \tau_{r \theta}= \pm \tau_{1}
$$

or

$$
\text { 2. } \begin{aligned}
\sigma_{r} & =\tau_{1} \sin \left(2 \theta+C_{1}\right)+C_{2}, \\
\sigma_{\theta} & =-\tau_{1} \sin \left(2 \theta+C_{1}\right)+C_{2}, \\
\tau_{r \theta} & =\tau_{1} \cos \left(2 \theta+C_{1}\right)
\end{aligned}
$$

Here, $C_{1}, C_{2}$ and $C_{3}$ are arbitrary constants. In Eq. (4.12), either the upper or lower sign should be taken. 


\subsection{Continuous Solution}

According to Eq. (4.6), it follows:

$$
\sigma_{\theta}=\tau_{1}, \tau_{r \theta}=\tau_{1} \quad(\text { in } \triangle \mathrm{OAB})
$$

The stresses $\sigma_{\theta}$ and $\tau_{r \theta}$ should be continuous along $\mathrm{OB}$. Then,

$$
\sigma_{\theta}=\tau_{1}, \tau_{r \theta}=\tau_{1} \quad \text { along } \theta=\frac{3 \pi}{4}(\mathrm{OB})
$$

From Eqs. (4.15) and (4.18) it follows:

$$
\begin{aligned}
& \sigma_{r}=\sigma_{\theta}=-2 \tau_{1} \theta+\tau_{1}\left(1+\frac{3 \pi}{2}\right) \\
& \tau_{r \theta}=\tau_{1}
\end{aligned}
$$

According to condition (4.5), the stresses $\sigma_{\theta}$ and $\tau_{r \theta}$ should be continuous along $\theta=\pi / 2(\mathrm{OC})$. Then from Eqs. (4.5), (4.15) and (4.19) it follows:

$$
\sigma_{r}=-2 \tau_{2} \theta+\pi\left(\tau_{2}-\tau_{1}\right)+\tau_{1}\left(1+\frac{3 \pi}{2}\right)
$$




$$
\begin{aligned}
& \left.\sigma_{\theta}=-2 \tau_{2} \theta+\pi\left(\tau_{2}-\tau_{1}\right)+\tau_{1}\left(1+\frac{3 \pi}{2}\right), \quad \text { (in } \Delta \mathrm{OCD}\right) \\
& \tau_{r \theta}=\tau_{1}
\end{aligned}
$$

From Eqs. (4.20) it can be seen that the plasticity condition is not met in the region OCD since $\tau_{r \theta}=\tau_{1}$ then Eq. (4.9) is not satisfied for $x>0$. However, for the homogeneous case when $\tau_{1}=\tau_{2}$, Eqs. (4.20) become

$$
\begin{aligned}
& \sigma_{r}=-2 \tau_{1} \theta+\tau_{1}\left(1+\frac{3 \pi}{2}\right), \\
& \sigma_{\theta}=-2 \tau_{1} \theta+\tau_{1}\left(1+\frac{3 \pi}{2}\right), \\
& \tau_{r \theta}=\tau_{1}
\end{aligned}
$$

and condition (4.9) is met everywhere.

In the triangle ODE it follows from Eq. (4.3) for the homogeneous case:

$$
\sigma_{x}=\sigma_{y}-2 \sqrt{\tau_{1}^{2}-\tau_{x y}^{2}} \quad(\Delta \mathrm{ODE})
$$

Assumed here is that $\sigma_{y}>\sigma_{x}$ in this triangle which is physically evident for opening mode tensile cracks. 
The stresses, $\sigma_{\theta}$ and $\tau_{r \theta}$, should be continuous along OD. Designate $\theta=\theta_{1}$ along OD. Expressing the stresses in the Cartesian coordinate system it follows that:

$$
\begin{aligned}
& -2 \tau_{1} \theta_{1}+\tau_{1}\left(1+\frac{3 \pi}{2}\right)=\sigma_{x} \sin ^{2} \theta_{1}+\sigma_{y} \cos ^{2} \theta_{1}- \\
& -2 \tau_{x y} \sin \theta_{1} \cos \theta_{1} \\
& \tau_{1}=\left(\sigma_{y}-\sigma_{x}\right) \sin \theta_{1} \cos \theta_{1}+\tau_{x y} \cos 2 \theta_{1} .
\end{aligned}
$$

Substituting Eq. (4.22) into (4.23), it follows:

$$
\begin{aligned}
& -2 \tau_{1} \theta_{1}+\tau_{1}\left(1+\frac{3 \pi}{2}\right)=\left(\sigma_{y}-2 \sqrt{\tau_{1}^{2}-\tau_{x y}^{2}}\right) \sin ^{2} \theta_{1}+ \\
& +\sigma_{y} \cos ^{2} \theta_{1}-2 \tau_{x y} \sin \theta_{1} \cos \theta_{1} \\
& \tau_{1}=\sqrt{\tau_{1}^{2}-\tau_{x y}^{2}} \sin 2 \theta_{1}+\tau_{x y} \cos 2 \theta_{1}
\end{aligned}
$$

There are two equations and the condition of $\tau_{x y}=0$ when $y=0$ and $x>0$ for determining $\theta_{1}$, and $\sigma_{y}$.

From Eq. (4.24) and the condition $\tau_{x y}=0, \theta_{1}$ can be found: 


$$
\theta_{1}=\frac{\pi}{4}
$$

From the equation system (4.24) the continuous solution for the homogeneous case can be derived:

$$
\begin{aligned}
& \sigma_{y}=-2 \tau_{1} \theta_{1}+\tau_{1}\left(1+\frac{3 \pi}{2}\right)+2 \tau_{1} \sin ^{2} \theta_{1} \\
& \sigma_{x}=-2 \tau_{1} \theta_{1}+\tau_{1}\left(1+\frac{3 \pi}{2}\right)-2 \tau_{1} \cos ^{2} \theta_{1} \quad(\Delta \mathrm{ODE}) \\
& \tau_{x y}=0
\end{aligned}
$$

From (4.26) and (4.25) it follows,

$$
\begin{array}{ll}
\sigma_{y} & =\tau_{1}(2+\pi), \\
\sigma_{x} & =\pi \tau_{1}, \\
\tau_{x y} & =0,
\end{array}
$$

Again, this is the first solution of the classical problem of the theory of plasticity obtained by Prandtl for the problem of a rigid smooth punch which as was shown by Hill and Sokolovsky, is mathematically identical to the crack problem (in homogeneous case). This solution, known as the Prandtl solution, coincides with that found previously in chapter 3. 


\subsection{Discontinuous Solution}

According to Eq. (4.6), it follows:

$$
\sigma_{\theta}=\tau_{1}, \tau_{r \theta}=\tau_{1} \quad(\text { in } \triangle \mathrm{OAB})
$$

The stresses $\sigma_{\theta}$ and $\tau_{r \theta}$ should be continuous along $\mathrm{OB}$. Then,

$$
\sigma_{\theta}=\tau_{1}, \tau_{r \theta}=\tau_{1} \quad \text { along } \theta=\frac{3 \pi}{4}(\mathrm{OB})
$$

From Eqs. (4.16) and (4.29) it can be found:

$$
\begin{array}{ll}
\sigma_{r} & =\tau_{1}(1+\cos 2 \theta), \\
\sigma_{\theta} & \left.=\tau_{1}(1-\cos 2 \theta), \quad \text { (in } \triangle \mathrm{OBC}\right) \\
\tau_{r \theta} & =-\tau_{1} \sin 2 \theta .
\end{array}
$$

According to condition (4.5), the stresses $\sigma_{\theta}$ and $\tau_{r \theta}$ should be continuous along $\theta=\pi / 2(\mathrm{OC})$. Then from Eqs. (4.5), (4.16) and (4.30) it follows: 


$$
\begin{aligned}
& \sigma_{r}=\tau_{2}(\cos 2 \theta-1)+2 \tau_{1}, \\
& \left.\sigma_{\theta}=-\tau_{2}(\cos 2 \theta+1)+2 \tau_{1}, \quad \text { (in } \triangle \mathrm{OCD}\right) \\
& \tau_{r \theta}=-\tau_{2} \sin 2 \theta .
\end{aligned}
$$

In the triangle ODE it follows from Eq. (4.3):

$$
\sigma_{x}=\sigma_{y}-2 \sqrt{\tau_{2}^{2}-\tau_{x y}^{2}}
$$

Assumed here is that $\sigma_{y}>\sigma_{x}$ in this triangle which is physically evident for opening mode tensile cracks.

The stresses, $\sigma_{\theta}$ and $\tau_{r \theta}$, should be continuous along OD. Designate $\theta=\theta_{2}$ along OD. Expressing the stresses in the Cartesian coordinate system it follows that:

$$
\begin{aligned}
& -\tau_{2}\left(\cos 2 \theta_{2}+1\right)+2 \tau_{1}=\sigma_{x} \sin ^{2} \theta_{2}+\sigma_{y} \cos ^{2} \theta_{2}- \\
& -2 \tau_{x y} \sin \theta_{2} \cos \theta_{2} \\
& -\tau_{2} \sin 2 \theta_{2}=\left(\sigma_{y}-\sigma_{x}\right) \sin \theta_{2} \cos \theta_{2}+\tau_{x y} \cos 2 \theta_{2}
\end{aligned}
$$

Substituting Eq. (4.32) into (4.33): 


$$
\begin{aligned}
& -\tau_{2}\left(\cos 2 \theta_{2}+1\right)+2 \tau_{1}=\left(\sigma_{y}-2 \sqrt{\tau_{2}^{2}-\tau_{x y}^{2}}\right) \sin ^{2} \theta_{2}+ \\
& +\sigma_{y} \cos ^{2} \theta_{2}-2 \tau_{x y} \sin \theta_{2} \cos \theta_{2} \\
& -\tau_{2} \sin 2 \theta_{2}=\sqrt{\tau_{2}^{2}-\tau_{x y}^{2}} \sin 2 \theta_{2}+\tau_{x y} \cos 2 \theta_{2}
\end{aligned}
$$

From the equation system (4.34) it follows:

$$
\begin{aligned}
& \sigma_{y}=\tau_{2}\left(2 \cos 4 \theta_{2} \sin ^{2} \theta_{2}-\sin 4 \theta_{2} \sin 2 \theta_{2}-\cos 2 \theta_{2}-1\right)+2 \tau_{1} \\
& \tau_{x y}=-\tau_{2} \sin 4 \theta_{2}
\end{aligned}
$$

Since $\tau_{x y}=0$ along $y=0, x>0$, substituting back into Eq. (4.35) $\theta_{2}$ can be determined. It follows from here that $\theta_{2}$ has the following solutions:

Case 1. $\theta_{2}=0 \quad$ for any $\lambda=\frac{\tau_{1}}{\tau_{2}}$

Case 2. $\theta_{2}=\frac{\pi}{4} \quad$ for any $\lambda=\frac{\tau_{1}}{\tau_{2}}$

Case 3.

$$
\theta_{2}=\frac{\pi}{2} \quad \text { for any } \lambda=\frac{\tau_{1}}{\tau_{2}}
$$

For the first and second case, from Eqs. (4.35) and (4.32) it follows that in these cases: 


$$
\left.\sigma_{y}=2\left(\tau_{1}-\tau_{2}\right), \quad \sigma_{x}=2\left(\tau_{1}-2 \tau_{2}\right), \quad \tau_{x y}=0 \quad \text { (in } \Delta \mathrm{ODE}\right)
$$

From Eqs. (4.39) $\sigma_{y}$ and $\sigma_{x}$ are compression stresses since $\tau_{1}<\tau_{2}(0 \leq \lambda \leq 1)$. Consequently, these two solutions have no physical meaning since we are considering the opening mode tensile crack where extension stresses are expected.

Considering the third case for $\theta_{2}=\pi / 2$, from Eqs. (4.35) and (4.32) it follows:

$$
\begin{array}{ll}
\sigma_{y} & =2\left(\tau_{1}+\tau_{2}\right), \\
\sigma_{x} & \left.=2 \tau_{1}, \quad \text { (in } \triangle \mathrm{ODE}\right) \\
\tau_{x y} & =0 .
\end{array}
$$

From here it can be concluded that points $\mathrm{C}$ and $\mathrm{D}$ in the original figure (Fig. 4.1) coincide in the characteristic line graph. Therefore, there is a unique region for $x>0$ with constant stresses with the values expressed in Eqs. (4.40). Equations (4.40) are valid for any $\lambda$ from 0 to 1 .

For the case of homogeneous material (Case 3) when $\tau_{1}=\tau_{2}$ from Eqs. (4.40) it follows: 


$$
\begin{aligned}
& \sigma_{y}=4 \tau_{1}, \\
& \sigma_{x}=2 \tau_{1}, \quad \text { when } \theta_{2}=\frac{\pi}{2} \quad(\triangle \mathrm{ODE}) \\
& \tau_{x y}=0
\end{aligned}
$$

This solution coincides with that found previously in Cherepanov (1997) [50]. The structure of the field of characteristic lines in this solution is shown in Fig.4.2b while the Fig. $4.2 \mathrm{a}$ gives the characteristic line structure in the classic Prandtl's solution.

From these results, it can be seen that this solution has a discontinuity in the stresses along the interface (line $\mathrm{OC}$ in Fig. 4.2b). In the triangle $\mathrm{OBC}$ the stresses are: $\sigma_{x}=2 \tau_{1}, \sigma_{y}=0$ and $\tau_{x y}=0$ while the stresses in the ODE region are: $\sigma_{x}=2 \tau_{1}, \sigma_{y}=2\left(\tau_{1}+\tau_{2}\right)$ and $\tau_{x y}=0$. The stresses in the $y$ direction are discontinuous. This result suggests that the velocity of the displacement along the interface is different which is reasonable in this type of problem.

\subsection{Velocity Distribution}

The characteristic lines of the Reuss equations for velocity coincide with those indicated above for stresses. Therefore, the velocity field is also formed by a combination of constant velocity fields and "fan" fields when: 


$$
v_{r}=0 \text { and } v_{\theta}=V_{0} \text { in "fan", }
$$

where $v_{r}$ and $v_{\theta}$ are the radial and tangential velocity components respectively ( $v_{r}=0$ because of a rigid radial constraint and $V_{0}$ is a constant specified by boundary conditions).

The results of simple calculations are given below:

1. The continuous solution:

$$
\left.v_{x}=\frac{\sqrt{2}}{2} V_{0}, \quad v_{y}=\frac{\sqrt{2}}{2} V_{0} \quad \text { (in } \quad \Delta \mathrm{OAB}, \text { Fig. } 4.2 \mathrm{a}\right)
$$

When $\theta=3 \pi / 4$, the tangential velocity is continuous. Then,

$$
\left.v_{x}=V_{0} \sin \theta, \quad v_{y}=-V_{0} \cos \theta \quad \text { (in } \Delta \mathrm{OBC}, \text { Fig. } 4.2 \mathrm{a}\right)
$$

Along the interface (line OC in Fig. 4.2a) the tangential velocity is continuous. Therefore, 


$$
v_{x}=V_{0} \cos \theta, \quad v_{y}=-V_{0} \cos \theta \quad \text { (in } \triangle \mathrm{OCD}, \text { Fig.4.2a) }
$$

When $\theta=\pi / 4$, the tangential velocity is continuous. Then,

$$
v_{x}=\frac{\sqrt{2}}{2} V_{0}, \quad v_{y}=-\frac{\sqrt{2}}{2} V_{0} \quad(\text { in } \Delta \mathrm{ODE}, \text { Fig.4.2a) }
$$

2. The discontinuous solution:

$$
\left.v_{x}=\frac{\sqrt{2}}{2} V_{0}, \quad v_{y}=\frac{\sqrt{2}}{2} V_{0} \quad \text { (in } \triangle \mathrm{OAB}, \text { and } \mathrm{OBC}, \text { Fig } 4.2 \mathrm{~b}\right)(
$$

Along the interface when $\theta=\pi / 2$, the tangential velocity is continuous. Therefore,

$$
v_{x}=\frac{\sqrt{2}}{2} V_{0}, \quad v_{y}=-\frac{\sqrt{2}}{2} V_{0} \quad \text { (in } \Delta \mathrm{ODE}, \text { Fig.4.2b) }
$$

From Eqs. (4.47) and (4.48) is observed that along the interface the velocity on the $x$ direction is continuous while the velocity on the $y$ direction is discontinuous. 


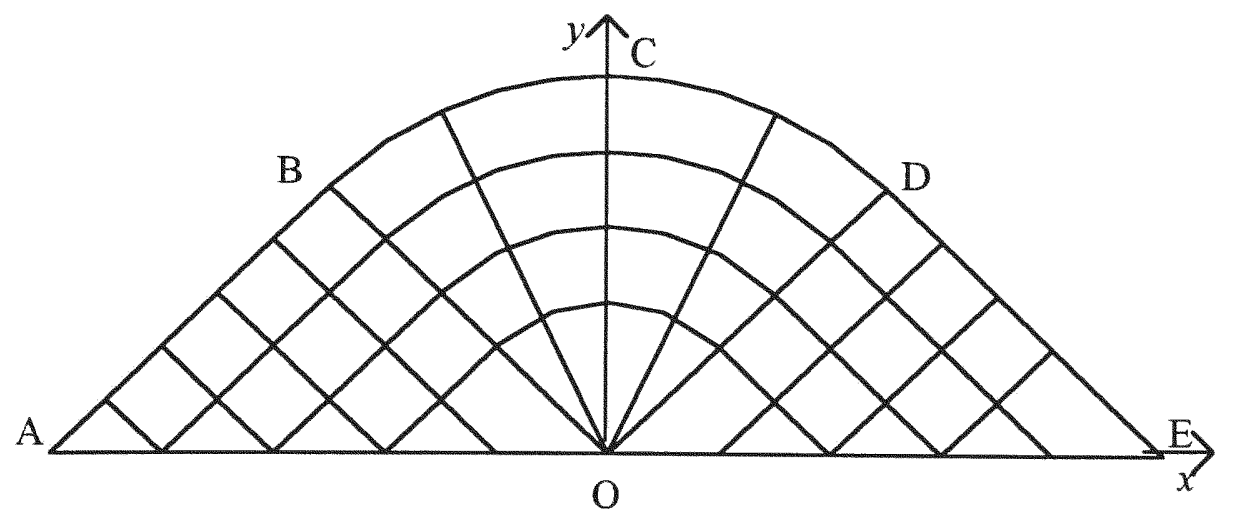

Fig 4.2a. Characteristic lines for the first solution of the plasticity theory. $\sigma_{y}=\tau_{1}(2+\pi), \sigma_{x}=\pi \tau_{1}, \tau_{x y}=0$ when $\theta_{2}=\frac{\pi}{4}$.

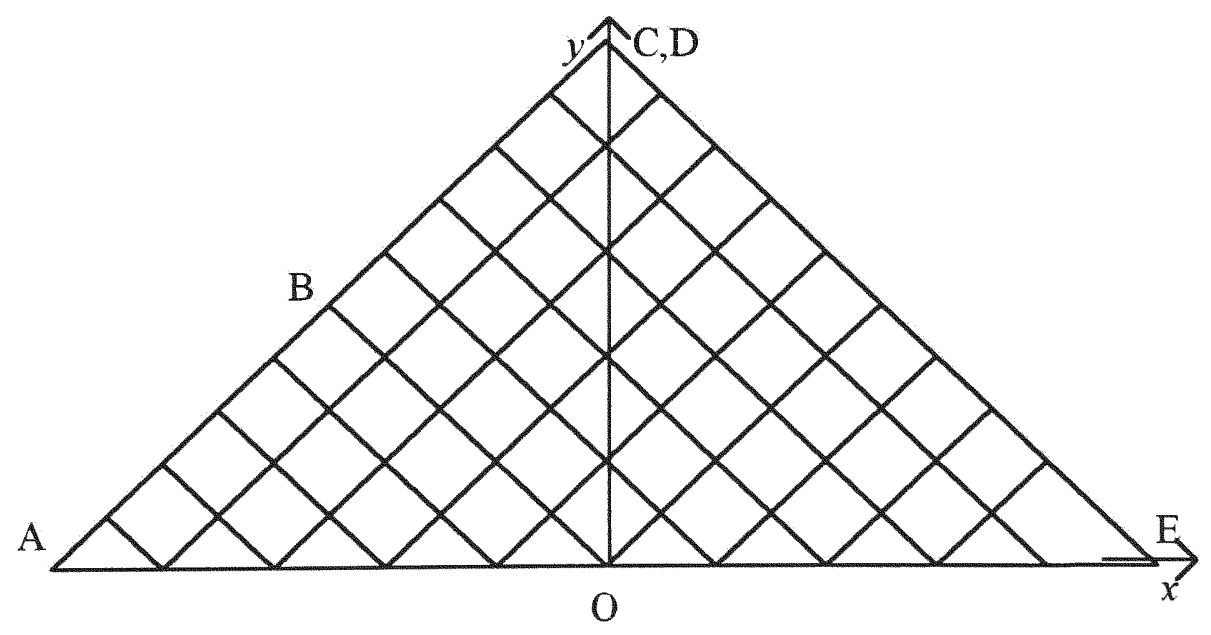

Fig 4.2b. Characteristic lines for the second solution of the plasticity theory.

$$
\sigma_{y}=4 \tau_{1}, \sigma_{x}=2 \tau_{1}, \tau_{x y}=0 \text { when } \theta_{2}=\frac{\pi}{2}
$$




\section{CHAPTER 5}

\section{A CRACK IMPINGING THE INTERFACE AT AN ARBITRARY ANGLE}

\subsection{Introduction}

In the last two chapters the problems for the case of an interface crack and the case of a crack impinging the interface at a right angle were considered. These can be considered as limiting cases for a more general problem of a crack impinging the interface of two different elastic-perfectly plastic materials at an arbitrary angle (see Fig. 5.1 on page 65). This chapter is devoted to the study of the latter general case. It will be shown that a feasible solution is found for the case when the arbitrary angle is greater than $45^{\circ}$. For the limiting case when the arbitrary angle is $90^{\circ}$, the solution coincides with that found previously in chapter 4 .

Again the materials are assumed here to be elastic-perfectly plastic solids. The plane strain condition is also assumed and the theory of shear lines or slip lines is applied to the solution of this problem. 


\subsection{Statement of the Problem}

Mathematically, the problem of stresses at the crack front is reduced to the following boundary value problem in plane strain:

$$
\begin{aligned}
& \frac{\partial \sigma_{x}}{\partial x}+\frac{\partial \tau_{x y}}{\partial y}=0 \quad(|x|<\infty,|y|<\infty), \\
& \frac{\partial \tau_{y x}}{\partial x}+\frac{\partial \sigma_{y}}{\partial y}=0 \quad(|x|<\infty,|y|<\infty), \\
& \left(\sigma_{y}-\sigma_{x}\right)^{2}+4 \tau_{x y}^{2}= \begin{cases}4 \tau_{1}^{2} & \text { for material \#1 } \\
4 \tau_{2}^{2} & \text { for material \#2}\end{cases} \\
& \sigma_{y}=\tau_{x y}=0 \quad \text { when } y=0, \quad x<0 \\
& {\left[\sigma_{\theta}\right]=\left[\tau_{r \theta}\right]=0 \text { along the interface. }}
\end{aligned}
$$

Equation (5.5) states that the tangential stress, $\sigma_{\theta}$, and the shear stress, $\tau_{r \theta}$, should be continuos along the interface.

As it has been considered in the previous chapters, the non-linear equation system (5.1) to (5.3) belongs to the hyperbolic type with two families of characteristic lines, that coincides with the slip lines, issuing from a free 
boundary under 45 degrees to the tangent at every point. From here, it follows that, in the rectangular equilateral triangle $O A B$ (see Fig. 5.1) having tractionfree crack banks as the diagonal, the only stress field is:

$$
\sigma_{y}=\tau_{x y}=0, \quad \sigma_{x}=2 \tau_{1} \quad \text { in } \quad \triangle \mathrm{OAB}
$$

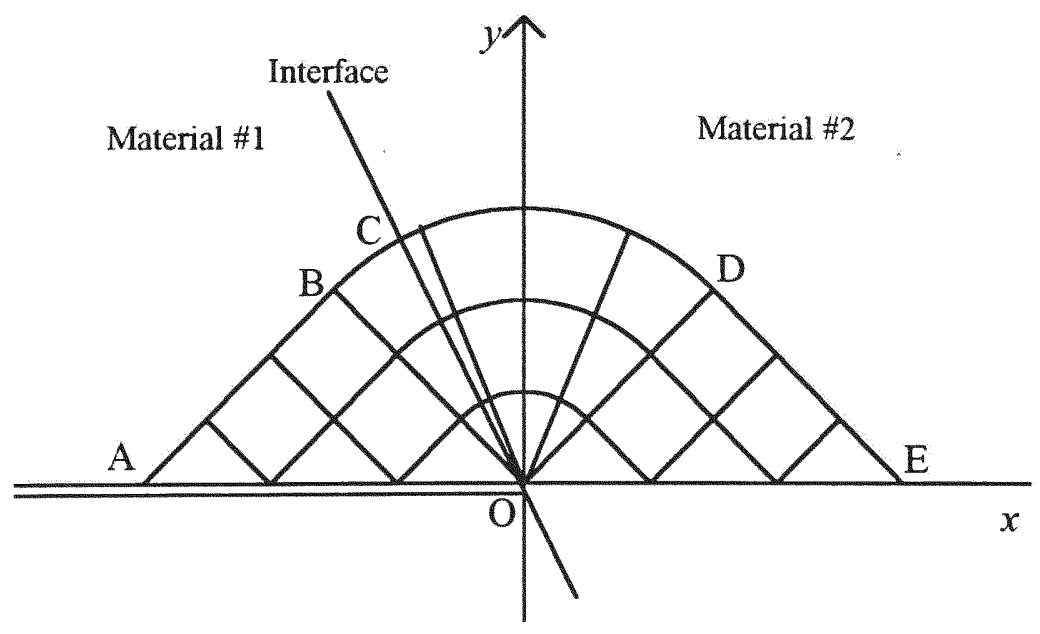

Fig. 5.1 The crack impinging the interface of two elastic-perfectly plastic materials bonded at an arbitrary angle.

Similar to the previous cases analyzed in chapters 3 and 4 the equations (4.7) and (4.8) in polar coordinates are the same for this case. The plasticity condition in polar coordinates becomes: 


$$
\left(\sigma_{r}-\sigma_{\theta}\right)^{2}+4 \tau_{r \theta}^{2}= \begin{cases}4 \tau_{1} & \text { for material \#1 } \\ 4 \tau_{2} & \text { for material \#2 }\end{cases}
$$

Equations (4.10) to (4.14) are valid for this problem. Therefore, the corresponding stress field for material \#1 is:

$$
\text { 1. } \sigma_{r}=\sigma_{\theta}=\mp 2 \tau_{1} \theta+C_{3}, \quad \tau_{r \theta}= \pm \tau_{1}
$$

or

$$
\text { 2. } \begin{aligned}
\sigma_{r} & =\tau_{1} \sin \left(2 \theta+C_{1}\right)+C_{2}, \\
\sigma_{\theta} & =-\tau_{1} \sin \left(2 \theta+C_{1}\right)+C_{2}, \\
\tau_{r \theta} & =\tau_{1} \cos \left(2 \theta+C_{1}\right)
\end{aligned}
$$

Here, $C_{1}, C_{2}$ and $C_{3}$ are arbitrary constants. In Eq. (5.8), either the upper or lower sign should be taken. By replacing $\tau_{1}$ with $\tau_{2}$, one can also get the corresponding stress field for material \#2.

The stress field for the continuous solution is that of Eq. (5.8) meanwhile the stress field for the discontinuous solution is that of Eq. (5.9). 
Let be $\varphi$ the arbitrary angle of the interface measured from line OA (see Fig. 5.2) in clockwise direction. Since there is a constant stress field in region $\mathrm{OAB}$ with a families of characteristic lines issuing from a free boundary under $45^{\circ}$, the cases when $\pi / 4<\varphi \leq \pi / 2$ will be considered in this study (see Fig. 5.2).

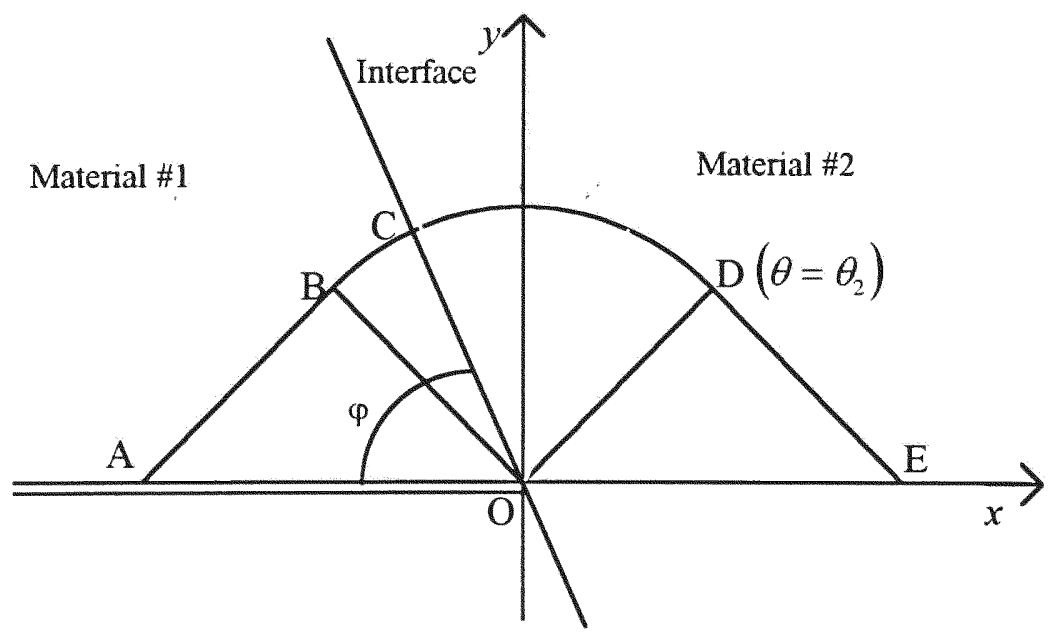

Fig. 5.2 The crack impinging an interface of two different elastic-plastic solids under an arbitrary angle $\varphi$ : the case when $\pi / 4 \leq \varphi \leq \pi / 2$. 


\subsection{Continuous Solution}

According to Eq. (5.6), it follows in triangle $\mathrm{OAB}$ :

$$
\sigma_{\theta}=\tau_{1}, \quad \tau_{r \theta}=\tau_{1} \quad(\text { in } \triangle \mathrm{OAB})
$$

The stresses $\sigma_{\theta}$ and $\tau_{r \theta}$ should be continuous along $\mathrm{OB}$. Then

$$
\sigma_{\theta}=\tau_{1}, \quad \tau_{r \theta}=\tau_{1} \quad \text { along } \quad \theta=\frac{3 \pi}{4} \quad(\mathrm{OC})
$$

From Eqs. (5.8) and (5.11) in $\triangle \mathrm{OBC}$ :

$$
\begin{aligned}
& \sigma_{r}=\sigma_{\theta}=-2 \tau_{1} \theta+\tau_{1}\left(1+\frac{3 \pi}{2}\right) \\
& \tau_{r \theta}=\tau_{1}
\end{aligned}
$$

According to condition (5.5), the stresses $\sigma_{\theta}$ and $\tau_{r \theta}$ should be continuous along the interface when $\theta=\pi-\varphi$ (OC). Then from Eqs. (5.8) and (5.12) it can be found in $\triangle O C D$ : 


$$
\begin{aligned}
& \sigma_{r}=\sigma_{\theta}=-2 \tau_{2} \theta+2(\pi-\varphi)\left(\tau_{2}-\tau_{1}\right)+\tau_{1}\left(1+\frac{3 \pi}{2}\right) \\
& \tau_{r \theta}=\tau_{1}
\end{aligned}
$$

From (5.13) it can be seen that the plasticity condition (Eq. (5.7)) is not met in the region OCD. Therefore, Eq. (5.13) is only valid in the case of a homogeneous material when $\tau_{1}=\tau_{2}$. Consequently, Eq. (5.3) becomes,

$$
\begin{aligned}
& \sigma_{r}=\sigma_{\theta}=-2 \tau_{1} \theta+\tau_{1}\left(1+\frac{3 \pi}{2}\right) \\
& \tau_{r \theta}=\tau_{1}
\end{aligned}
$$

Then, similar to the case study in chapter 4 for the continuous case the following solution is found in triangle ODE

$$
\sigma_{y}=\tau_{1}(2+\pi), \quad \sigma_{x}=\pi \tau_{1}, \quad \tau_{x y}=0, \quad \text { when } \theta_{2}=\frac{\pi}{4}
$$

This solution coincides with the result found previously by Prandtl which as was shown by Hill and Sokolovsky, is mathematically identical to the crack problem in homogeneous case. 


\subsection{Discontinuous Solution}

Again, according to Eq. (5.6) it follows in triangle OAB:

$$
\left.\sigma_{\theta}=\tau_{1}, \quad \tau_{r \theta}=\tau_{1} \quad \text { (in } \triangle \mathrm{OAB}\right)
$$

The stresses $\sigma_{\theta}$ and $\tau_{r \theta}$ should be continuous along $\mathrm{OB}$. Then

$$
\sigma_{\theta}=\tau_{1}, \quad \tau_{r \theta}=\tau_{1} \quad \text { along } \quad \theta=\frac{3 \pi}{4} \quad(\mathrm{OC})
$$

From Eqs. (5.9) and (5.17) it can be found in $\triangle O B C$ :

$$
\begin{aligned}
& \sigma_{r}=\tau_{1}(1+\cos 2 \theta) \\
& \sigma_{\theta}=\tau_{1}(1-\cos 2 \theta) \\
& \tau_{r \theta}=-\tau_{1} \sin 2 \theta
\end{aligned}
$$

According to condition (5.5), the stresses $\sigma_{\theta}$ and $\tau_{r \theta}$ should be continuous along the interface when $\theta=\pi-\varphi(\mathrm{OC})$. Then from Eqs. (5.9) and (5.18) it can be found in $\triangle O C D$ : 


$$
\begin{aligned}
& \sigma_{r}=\tau_{2} \sin \left(2 \theta+C_{1}\right)+\tau_{1}(1-\cos 2 \varphi)+\tau_{2} \sin \left(C_{1}-2 \varphi\right) \\
& \sigma_{\theta}=-\tau_{2} \sin \left(2 \theta+C_{1}\right)+\tau_{1}(1-\cos 2 \varphi)+\tau_{2} \sin \left(C_{1}-2 \varphi\right) \\
& \tau_{r \theta}=\tau_{2} \cos \left(2 \theta+C_{1}\right)
\end{aligned}
$$

where

$$
C_{1}=2 \varphi-\arccos (\lambda \sin 2 \varphi), \quad \text { and } \quad \lambda=\frac{\tau_{1}}{\tau_{2}}
$$

$C_{1}$ can be found numerically. Its solution is shown in Table 5.1.

In the triangle ODE it follows:

$$
\sigma_{x}=\sigma_{y}-2 \sqrt{\tau_{2}^{2}-\tau_{x y}^{2}}
$$

Assumed here is that $\sigma_{y}>\sigma_{x}$ in this triangle which is physically evident for opening mode tensile cracks.

The stresses, $\sigma_{\theta}$ and $\tau_{r \theta}$ should be continuous along OD. Designate $\theta=\theta_{2}$ along OD. Expressing the stresses in the Cartesian coordinate system: 
Table 5.1. Numerical solution of equation (5.20)

\begin{tabular}{|c|c|c|c|c|c|c|c|c|c|c|c|}
\hline$\lambda=\frac{\tau_{1}}{\tau_{2}}$ & 0 & 0.1 & 0.2 & 0.3 & 0.4 & 0.5 & 0.6 & 0.7 & 0.8 & 0.9 & 1.0 \\
\hline$\varphi$ & & & & & & $C_{1}$ & & & & & \\
\hline 45 & 0 & 5.7319 & 11.536 & 17.457 & 23.578 & 30 & 36.869 & 44.427 & 53.13 & 64.158 & 90 \\
\hline 50 & 10 & 15.651 & 21.359 & 27.184 & 33.198 & 39.498 & 46.219 & 53.579 & 61.984 & 72.415 & 90 \\
\hline 55 & 20 & 25.392 & 30.832 & 36.374 & 42.078 & 48.024 & 54.320 & 61.131 & 68.742 & 77.749 & 90 \\
\hline 60 & 30 & 34.968 & 39.974 & 45.058 & 50.267 & 55.658 & 61.306 & 67.316 & 73.853 & 81.207 & 90 \\
\hline 65 & 40 & 44.393 & 48.812 & 53.286 & 57.843 & 62.521 & 67.363 & 72.427 & 77.794 & 83.585 & 90 \\
\hline 70 & 50 & 53.685 & 57.386 & 61.118 & 64.898 & 68.747 & 72.685 & 76.74 & 80.946 & 85.345 & 90 \\
\hline 75 & 60 & 62.865 & 65.739 & 68.629 & 71.536 & 74.477 & 77.457 & 80.487 & 83.578 & 86.743 & 90 \\
\hline 80 & 70 & 71.96 & 73.922 & 75.889 & 77.863 & 79.846 & 81.841 & 83.851 & 85.879 & 87.927 & 90 \\
\hline 85 & 80 & 80.994 & 81.990 & 82.986 & 83.982 & 84.980 & 85.980 & 86.918 & 87.985 & 88.991 & 90 \\
\hline 90 & 90 & 90 & 90 & 90 & 90 & 90 & 90 & 90 & 90 & 90 & 90 \\
\hline
\end{tabular}




$$
\begin{aligned}
& -\tau_{2} \sin \left(2 \theta_{2}+C_{1}\right)+\tau_{1}(1-\cos 2 \varphi)+\tau_{2} \sin \left(C_{1}-2 \varphi\right)=\sigma_{x} \sin ^{2} \theta_{2}+ \\
& +\sigma_{y} \cos ^{2} \theta_{2}-2 \tau_{x y} \sin \theta_{2} \cos \theta_{2} \\
& \tau_{2} \cos \left(2 \theta_{2}+C_{1}\right)=\left(\sigma_{y}-\sigma_{x}\right) \sin \theta_{2} \cos \theta_{2}+\tau_{x y} \cos 2 \theta_{2}
\end{aligned}
$$

From Eqs. (5.21) and (5.22) it follows:

$$
\begin{aligned}
& -\tau_{2} \sin \left(2 \theta_{2}+C_{1}\right)+\tau_{1}(1-\cos 2 \varphi)+\tau_{2} \sin \left(C_{1}-2 \varphi\right)= \\
& \left.=\left(\sigma_{y}-2 \sqrt{\tau_{2}^{2}-\tau_{x y}^{2}}\right) \sin ^{2} \theta_{2}+\sigma_{y} \cos ^{2} \theta_{2}-2 \tau_{x y} \sin \theta_{2} \cos \theta_{2} \quad \text { (in } \Delta \mathrm{ODE}\right) \\
& \tau_{2} \cos \left(2 \theta_{2}+C_{1}\right)=\sqrt{\tau_{2}^{2}-\tau_{x y}^{2}} \sin 2 \theta_{2}+\tau_{x y} \cos 2 \theta_{2} .
\end{aligned}
$$

From the equation system (5.23) and the condition of $\tau_{x y}=0$ along $y=0, x>0$, $\theta_{2}$ can be determined. The numerical solution for $\theta_{2}$ is shown in Table 5.2.

From equation system (5.23) and equation (5.21) we have in triangle ODE:

$$
\begin{aligned}
& \sigma_{y}=-\tau_{2} \sin \left(2 \theta_{2}+C_{1}\right)+\tau_{1}(1-\cos 2 \varphi)+\tau_{2} \sin \left(C_{1}-2 \varphi\right)+2 \tau_{2} \sin ^{2} \theta_{2} \\
& \sigma_{x}=-\tau_{2} \sin \left(2 \theta_{2}+C_{1}\right)+\tau_{1}(1-\cos 2 \varphi)+\tau_{2} \sin \left(C_{1}-2 \varphi\right)-2 \tau_{2} \cos ^{2} \theta_{2}
\end{aligned}
$$

The equation system (5.24) can be easily solved numerically. The solution of this is shown in Table 5.3a and Table 5.3b and Figs. 5.3 and 5.4 (some values of $\varphi$ ) for $\sigma_{y} / \tau_{2}$ and $\sigma_{x} / \tau_{z}$ respectively. 
Table 5.2. Numerical solution for $\theta_{2}$.

\begin{tabular}{|c|c|c|c|c|c|c|c|c|c|c|c|c|}
\hline$\lambda=\tau_{1} / \tau_{2}$ & 0 & 0.1 & 0.2 & 0.3 & 0.4 & 0.5 & 0.6 & 0.7 & 0.8 & 0.9 & 1.0 \\
\hline$\varphi$ & & \multicolumn{7}{|c|}{$\theta_{2}$} \\
\hline 45 & 112.5 & 111.06 & 109.61 & 108.13 & 106.6 & 105 & 103.28 & 101.39 & 99.217 & 96.46 & 90 \\
\hline 50 & 110 & 108.58 & 107.16 & 105.7 & 104.2 & 102.62 & 100.94 & 99.105 & 97.003 & 94.396 & 90 \\
\hline 55 & 107.5 & 106.15 & 104.79 & 103.40 & 101.98 & 100.49 & 98.919 & 97.217 & 95.314 & 93.062 & 90 \\
\hline 60 & 105 & 103.75 & 102.5 & 101.23 & 99.933 & 98.585 & 97.173 & 95.67 & 94.036 & 92.198 & 90 \\
\hline 65 & 102.5 & 101.40 & 100.29 & 99.178 & 98.039 & 96.869 & 95.659 & 94.393 & 93.051 & 91.603 & 90 \\
\hline 70 & 100 & 99.078 & 98.153 & 97.22 & 96.275 & 95.313 & 94.328 & 93.314 & 92.263 & 91.163 & 90 \\
\hline 75 & 97.5 & 96.783 & 96.065 & 95.343 & 94.615 & 93.880 & 93.135 & 92.378 & 91.605 & 90.814 & 90 \\
\hline 80 & 95 & 94.51 & 94.019 & 93.527 & 93.034 & 92.538 & 92.039 & 91.537 & 91.03 & 90.518 & 90 \\
\hline 85 & 92.5 & 92.251 & 92.002 & 91.753 & 91.504 & 91.254 & 91.004 & 90.754 & 90.503 & 90.252 & 90 \\
\hline 90 & 90 & 90 & 90 & 90 & 90 & 90 & 90 & 90 & 90 & 90 & 90 \\
\hline
\end{tabular}


Table 5.3a. Numerical solution of the equation system (5.24) for $\frac{\sigma_{y}}{\tau_{2}}$.

\begin{tabular}{|c|c|c|c|c|c|c|c|c|c|c|c|c|c|}
\hline$\lambda=\tau_{1} / \tau_{2}$ & \multicolumn{1}{|c|}{0.1} & 0.2 & 0.3 & 0.4 & 0.5 & 0.6 & 0.7 & 0.8 & 0.9 & 1.0 \\
\hline$\varphi$ & \multicolumn{10}{|c|}{$\sigma_{y} / \tau_{2}$} \\
\hline 45 & 1.4142 & 1.5882 & 1.7693 & 1.9585 & 2.1568 & 2.366 & 2.5888 & 2.8297 & 3.0973 & 3.4134 & 4 \\
\hline 50 & 1.5320 & 1.7158 & 1.9061 & 2.1036 & 2.3096 & 2.5253 & 2.7532 & 2.9969 & 3.2635 & 3.5697 & 4 \\
\hline 55 & 1.6383 & 1.8290 & 2.0254 & 2.2281 & 2.4377 & 2.6555 & 2.8831 & 3.1230 & 3.3798 & 3.6627 & 4 \\
\hline 60 & 1.7320 & 1.9275 & 2.1275 & 2.3324 & 2.5428 & 2.7594 & 2.9832 & 3.2156 & 3.4590 & 3.7176 & 4 \\
\hline 65 & 1.8126 & 2.0108 & 2.2125 & 2.4178 & 2.6269 & 2.8404 & 3.0586 & 3.2824 & 3.5126 & 3.7510 & 4 \\
\hline 70 & 1.8793 & 2.0790 & 2.2810 & 2.4853 & 2.6922 & 2.9017 & 3.1142 & 3.3298 & 3.5489 & 3.7721 & 4 \\
\hline 75 & 1.9318 & 2.1320 & 2.3335 & 2.5364 & 2.7407 & 2.9464 & 3.1537 & 3.3625 & 3.5731 & 3.7855 & 4 \\
\hline 80 & 1.9696 & 2.1698 & 2.3706 & 2.5720 & 2.7740 & 2.9767 & 3.1800 & 3.3839 & 3.5886 & 3.7939 & 4 \\
\hline
\end{tabular}


Table 5.3b. Numerical solution of the equation system (5.24) for $\frac{\sigma_{x}}{\tau_{2}}$.

\begin{tabular}{|c|c|c|c|c|c|c|c|c|c|c|c|c|}
\hline$\lambda=\tau_{1} / \tau_{2}$ & 0 & 0.1 & 0.2 & 0.3 & 0.4 & 0.5 & 0.6 & 0.7 & 0.8 & 0.9 & 1.0 \\
\hline$\varphi$ & \multicolumn{9}{|c|}{$\sigma_{x} / \tau_{2}$} \\
\hline 45 & -0.585 & -0.411 & -0.230 & -0.041 & 0.1568 & 0.3660 & 0.5888 & 0.8297 & 1.0973 & 1.4134 & 2 \\
\hline 50 & -0.467 & -0.284 & -0.093 & 0.1036 & 0.3096 & 0.5253 & 0.7532 & 0.9969 & 1.2635 & 1.5697 & 2 \\
\hline 55 & -0.361 & -0.170 & 0.0254 & 0.2281 & 0.4377 & 0.6555 & 0.8831 & 1.1230 & 1.3798 & 1.6620 & 2 \\
\hline 60 & -0.267 & -0.072 & 0.1275 & 0.3324 & 0.5428 & 0.7594 & 0.9832 & 1.2156 & 1.4590 & 1.7176 & 2 \\
\hline 65 & -0.187 & 0.0108 & 0.2125 & 0.4178 & 0.6269 & 0.8404 & 1.0586 & 1.2824 & 1.5126 & 1.7510 & 2 \\
\hline 70 & -0.120 & 0.0790 & 0.2810 & 0.4853 & 0.6922 & 0.9017 & 1.1142 & 1.3298 & 1.5489 & 1.7721 & 2 \\
\hline 75 & -0.068 & 0.1320 & 0.3335 & 0.5364 & 0.7407 & 0.9464 & 1.1537 & 1.3625 & 1.5731 & 1.7855 & 2 \\
\hline 80 & -0.030 & 0.1698 & 0.3706 & 0.5720 & 0.7740 & 0.9767 & 1.1800 & 1.3839 & 1.5886 & 1.7939 & 2 \\
\hline 85 & -0.007 & 0.1924 & 0.3926 & 0.5930 & 0.7935 & 0.9942 & 1.1950 & 1.3960 & 1.5972 & 1.7985 & 2 \\
\hline 90 & 0 & 0.2 & 0.4 & 0.6 & 0.8 & 1.0 & 1.2 & 1.4 & 1.6 & 1.8 & 2 \\
\hline
\end{tabular}




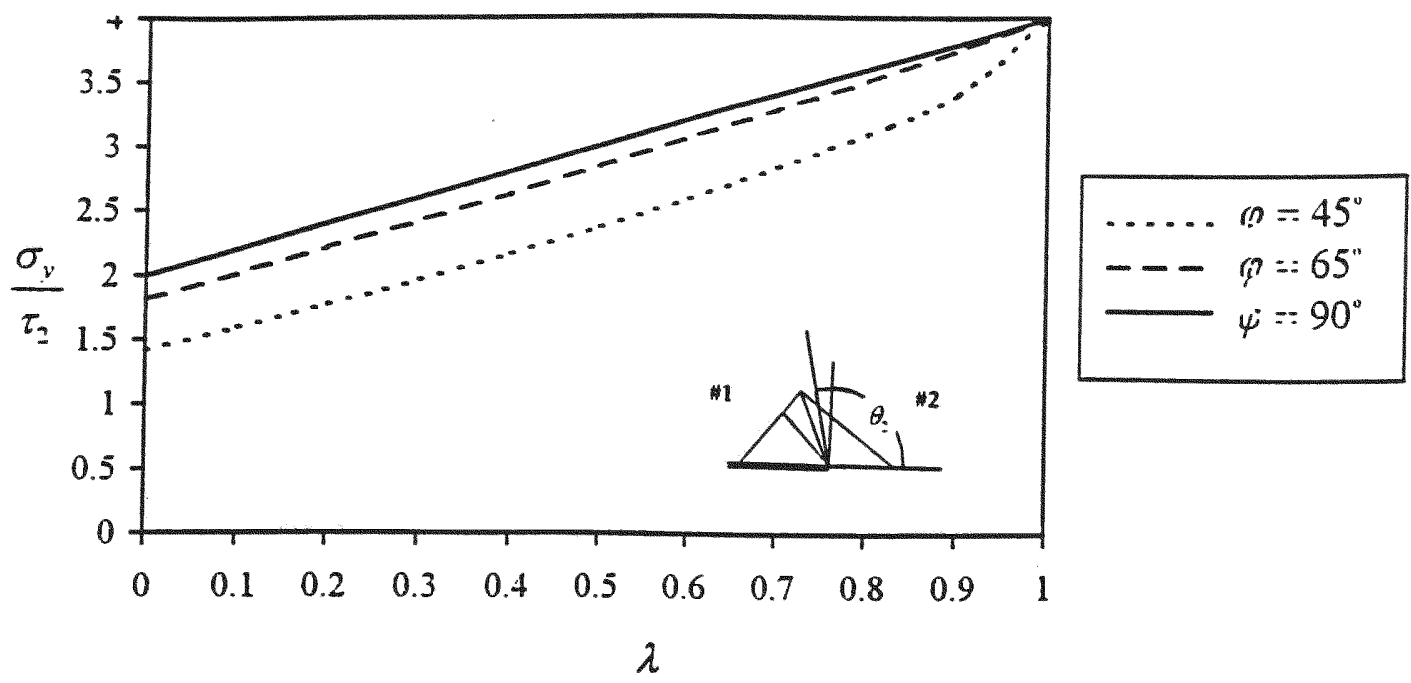

Fig $5.3 \mathrm{Graph} \frac{\sigma_{y}}{\tau_{2}}$ vs. $\lambda$ for discontinuous solution in the case of a crack impinging an interface at arbitrary angle. The $\sigma_{y}$ is the corresponding stress for $\theta_{2}>\theta>0$.

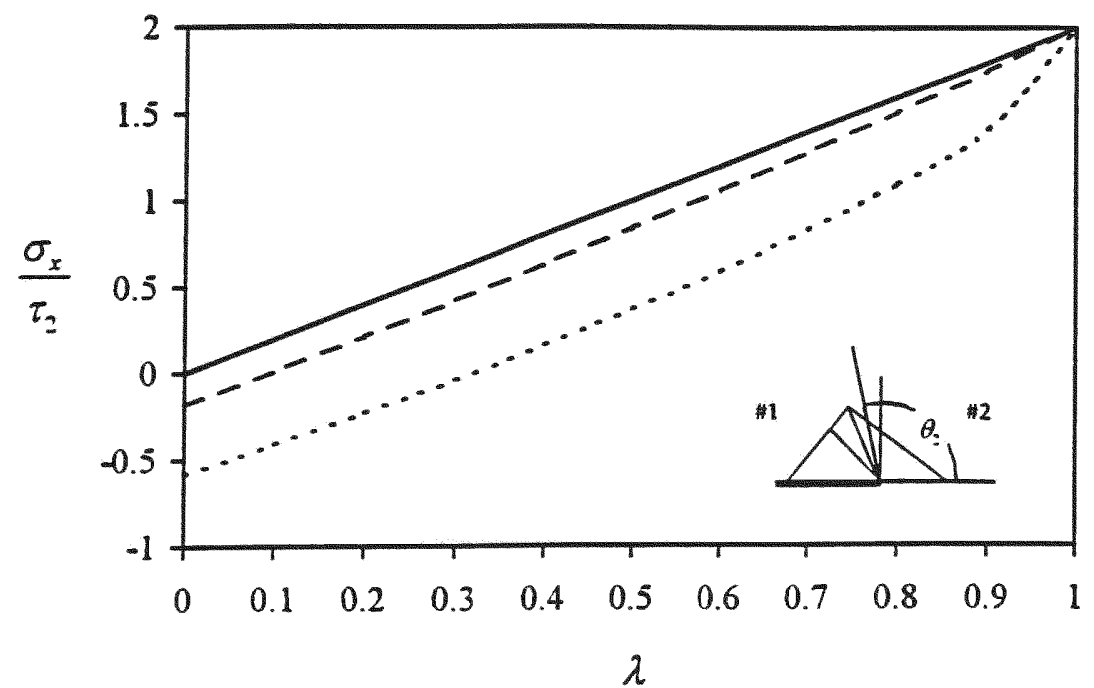

$$
\begin{aligned}
\ldots \ldots & \varphi=45^{\circ} \\
-- & \psi \\
- & =65^{\circ} \\
\psi & =90^{\circ}
\end{aligned}
$$

Fig 5.4 Graph $\frac{\sigma_{x}}{\tau_{z}}$ vs. $\lambda$ for discontinuous solution in the case of a crack impinging an interface at an arbitrary angle. The $\sigma_{x}$ is the corresponding stress for $\theta_{2}>\theta>0$. 
From the Eq. (5.20) it can be seen that when $\varphi=\pi / 2$ then $C_{1}=\pi / 2$ and $\theta_{2}=\pi / 2$. Consequently, the equation system (5.24) becomes:

$$
\begin{aligned}
& \sigma_{y}=2\left(\tau_{1}+\tau_{2}\right) \\
& \sigma_{x}=2 \tau_{1} \\
& \tau_{x y}=0
\end{aligned}
$$

From Eq. (5.25), it can be seen that for the case of homogeneous material when $\tau_{1}=\tau_{2}$, it follows

$$
\begin{aligned}
& \sigma_{y}=4 \tau_{1}, \\
& \sigma_{x}=2 \tau_{1}, \quad \text { when } \quad \theta_{2}=\frac{\pi}{2} \\
& \tau_{x y}=0
\end{aligned}
$$

This solution coincides with that found previously in Cherepanov [50] for this particular case.

\subsection{Velocity Distribution}

The characteristic of the stresses and velocity coincides; therefore, using the Geiringer velocity equations and introducing the following velocity components, 


$$
v_{r}=0 \text { and } v_{\theta}=V_{0} \text { in "fan", }
$$

where $v_{r}$ and $v_{\theta}$ are the radial and tangential velocity components respectively ( $v_{r}=0$ because of a rigid radial constraint and $V_{0}$ is a constant specified by boundary conditions).

The results for the continuous solution are the same of those expressed in Eqs. (4.43) to (4.46) in the previous chapter.

In the discontinuous case, the velocity components coincide with those expressed in Eqs. (4.47) and (4.48). However, in this general case for an arbitrary angle, the region $\mathrm{OCD}$ (Fig. 5.2) should be included and the velocity components are the same of those of Eq. (4.48). From here it can be seen that velocity components are continuous along the line OD in Fig. 5.1 but discontinuity appears along the interface (line OC Fig. 5.1). 


\section{CHAPTER 6}

\section{PROBLEM OF SMOOTH RIGID PUNCH}

\subsection{Introduction}

In the previous chapters the states of stress inside the plastic region at the crack tip for an inhomogeneous material have been studied when the crack is along the interface or impinging the interface of two different elastic-perfectly plastic solids. However, according to Hill [11] and Sokolovsky [12], the stresses at the crack tip in the case of a homogeneous material coincide with those at the punch edge discovered earlier by Prandtl [8]. Analogously, this idea can be extended for the case of an inhomogeneous material subjected to a punch force as shown in Fig. 6.1. Therefore, in this chapter the problem of the indentation of a semiinfinite inhomogeneous body by a flat rigid punch in the form of an infinite strip is studied (see Fig. 6.1 on Page 81).

\subsection{Problem Statement}

It is assumed that the surface of the punch and body are perfectly lubricated, so that there is no friction between them (smooth surface line OE in Fig. 6.1). The constituent materials are also assumed elastic-perfectly plastic material and the theory of shear lines or slip lines is used in the same way that it was used in the 
previous chapters. In this case the stresses found here can be used to determine the limiting load.

Mathematically, the problem of stresses at the punch surface is identical to the boundary value problem of stresses at the crack tip front in plane strain. Consequently, Eqs. (4.1) to (4.5) are valid in this case. Surface OE in Fig. 6.1 is free of traction then $\sigma_{y}=\tau_{x y}=0$.

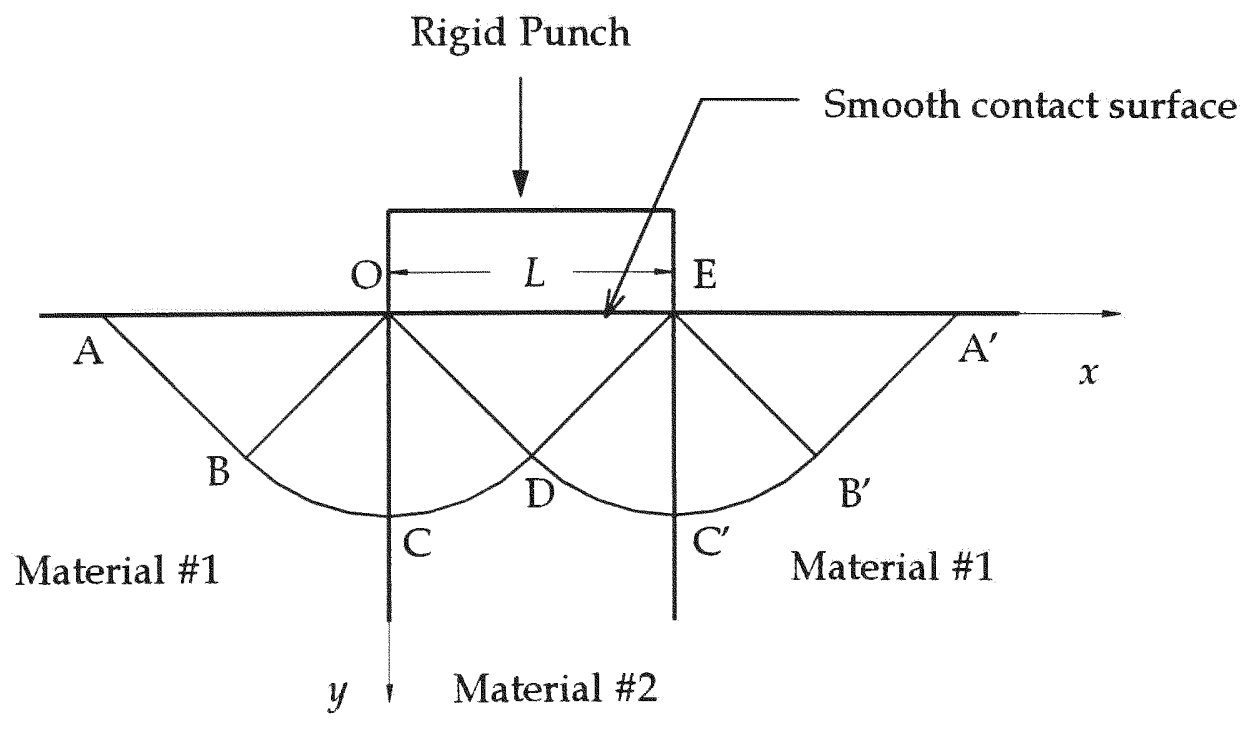

Fig. 6.1 The smooth rigid punch problem for an inhomogeneous material. 
In region $\mathrm{OAB}$ (Fig. 6.1) the only stress field is:

$$
\sigma_{y}=\tau_{x y}=0, \quad \sigma_{x}=2 \tau_{1} \quad \text { in } \quad \triangle \mathrm{OAB}
$$

Equilibrium and yielding condition equations in polar coordinates are the same of those expressed in Eqs. (4.7) to (4.9).

Assuming the function $f(\theta)$ in the form of Eq. (4.10) and substituting back into (4.7) and (4.8) two possible solutions are also found for the stress field:

1. $\sigma_{r}=\sigma_{\theta}=\mp 2 \tau_{1} \theta+C_{3}, \quad \tau_{r \theta}= \pm \tau_{1}$

or

2. $\sigma_{r}=\tau_{1} \sin \left(2 \theta+C_{1}\right)+C_{2}$,

$$
\begin{aligned}
& \sigma_{\theta}=-\tau_{1} \sin \left(2 \theta+C_{1}\right)+C_{2}, \\
& \tau_{r \theta}=\tau_{1} \cos \left(2 \theta+C_{1}\right)
\end{aligned}
$$

\subsection{Continuous Solution}

According to Eq. (4.6), it follows: 


$$
\sigma_{\theta}=\tau_{1}, \tau_{r \theta}=\tau_{1} \quad(\text { in } \quad \Delta \mathrm{OAB})
$$

The stresses $\sigma_{\theta}$ and $\tau_{r \theta}$ should be continuous along $\mathrm{OB}$. Then,

$$
\sigma_{\theta}=\tau_{1}, \quad \tau_{r \theta}=\tau_{1} \quad \text { along } \theta=\frac{3 \pi}{4}
$$

From Eqs. (4.15) and (6.5) it follows:

$$
\begin{aligned}
& \left.\sigma_{r}=\sigma_{\theta}=-2 \tau_{1} \theta+\tau_{1}\left(1+\frac{3 \pi}{2}\right) \quad \text { (in } \triangle \mathrm{OBC}\right) \\
& \tau_{r \theta}=\tau_{1}
\end{aligned}
$$

According to condition (4.5), the stresses $\sigma_{\theta}$ and $\tau_{r \theta}$ should be continuous along $\theta=\pi / 2(\mathrm{OC})$. This condition and the plasticity condition (Eq. (4.9) are met only for the homogeneous case when $\tau_{1}=\tau_{2}$,

$$
\begin{aligned}
& \sigma_{r}=-2 \tau_{1} \theta+\tau_{1}\left(1+\frac{3 \pi}{2}\right), \\
& \left.\sigma_{\theta}=-2 \tau_{1} \theta+\tau_{1}\left(1+\frac{3 \pi}{2}\right), \quad \text { (in } \triangle \mathrm{OCD}\right) \\
& \tau_{r \theta}=\tau_{1}
\end{aligned}
$$


In the triangle ODE it follows from Eq. (4.3) for the homogeneous case:

$$
\sigma_{x}=\sigma_{y}-2 \sqrt{\tau_{1}^{2}-\tau_{x y}^{2}}
$$

Assumed here is that $\sigma_{y}>\sigma_{x}$ in this triangle which is physically evident for opening mode tensile cracks.

The stresses, $\sigma_{\theta}$ and $\tau_{r \theta}$, should be continuous along OD. Designate $\theta=-\theta_{1}$ along OD. Expressing the stresses in the Cartesian coordinate system it follows that:

$$
\begin{aligned}
& -2 \tau_{1} \theta_{1}+\tau_{1}\left(1+\frac{3 \pi}{2}\right)=\left(\sigma_{y}-2 \sqrt{\tau_{1}^{2}-\tau_{x y}^{2}}\right) \sin ^{2} \theta_{1}+ \\
& +\sigma_{y} \cos ^{2} \theta_{1}-2 \tau_{x y} \sin \theta_{1} \cos \theta_{1} \\
& \tau_{1}=\sqrt{\tau_{1}^{2}-\tau_{x y}^{2}} \sin 2 \theta_{1}+\tau_{x y} \cos 2 \theta_{1}
\end{aligned}
$$

There are two equations and the condition of $\tau_{x y}=0$ when $y=0$ and $x>0$ (smooth contact surface $\mathrm{OE}$ ) for determining $\theta_{1}$, and $\sigma_{y}$.

From Eq. (4.9) and the condition $\tau_{x y}=0, \theta_{1}$ can be found: 


$$
\theta_{1}=\frac{\pi}{4}
$$

From the equation system (6.9) the continuous solution for the homogeneous case can be derived,

$$
\begin{aligned}
& \sigma_{y}=\tau_{1}(2+\pi), \\
& \sigma_{x}=\pi \tau_{1}, \quad \text { when } \theta_{1}=\frac{\pi}{4} . \\
& \tau_{x y}=0,
\end{aligned}
$$

This is the first solution of the classical problem of the theory of plasticity obtained by Prandtl for the problem of a rigid smooth punch which as was shown by Hill and Sokolovsky, is mathematically identical to the crack problem (in homogeneous case).

\subsection{Discontinuous Solution}

According to Eq. (4.6), it follows:

$$
\sigma_{\theta}=\tau_{1}, \tau_{r \theta}=\tau_{1} \quad(\text { in } \triangle \mathrm{OAB})
$$


The stresses $\sigma_{\theta}$ and $\tau_{r \theta}$ should be continuous along $\mathrm{OB}$. Then,

$$
\sigma_{\theta}=\tau_{1}, \quad \tau_{r \theta}=\tau_{1} \quad \text { along } \theta=\frac{3 \pi}{4}(\mathrm{OB})
$$

From Eqs. (4.16) and (6.13) it can be found:

$$
\begin{array}{ll}
\sigma_{r} & =\tau_{1}(1+\cos 2 \theta), \\
\sigma_{\theta} & =\tau_{1}(1-\cos 2 \theta), \quad \quad(\text { in } \triangle \mathrm{OBC}) \\
\tau_{r \theta} & =-\tau_{1} \sin 2 \theta .
\end{array}
$$

According to condition (4.5), the stresses $\sigma_{\theta}$ and $\tau_{r \theta}$ should be continuous along $\theta=\pi / 2(\mathrm{OC})$. Then from Eqs. (4.5), (4.16) and (6.14) it follows:

$$
\begin{aligned}
& \sigma_{r}=\tau_{2}(\cos 2 \theta-1)+2 \tau_{1}, \\
& \left.\sigma_{\theta}=-\tau_{2}(\cos 2 \theta+1)+2 \tau_{1}, \quad \text { (in } \triangle O C D\right) \\
& \tau_{r \theta}=-\tau_{2} \sin 2 \theta .
\end{aligned}
$$

In the triangle ODE it follows from Eq. (4.3): 


$$
\sigma_{x}=\sigma_{y}-2 \sqrt{\tau_{2}^{2}-\tau_{x y}^{2}}
$$

The stresses, $\sigma_{\theta}$ and $\tau_{r \theta}$, should be continuous along OD. Designate $\theta=\theta_{2}$ along OD. Expressing the stresses in the Cartesian coordinate system it follows that:

$$
\begin{aligned}
& -\tau_{2}\left(\cos 2 \theta_{2}+1\right)+2 \tau_{1}=\left(\sigma_{y}-2 \sqrt{\tau_{2}^{2}-\tau_{x y}^{2}}\right) \sin ^{2} \theta_{2}+ \\
& +\sigma_{y} \cos ^{2} \theta_{2}-2 \tau_{x y} \sin \theta_{2} \cos \theta_{2} \\
& -\tau_{2} \sin 2 \theta_{2}=\sqrt{\tau_{2}^{2}-\tau_{x y}^{2}} \sin 2 \theta_{2}+\tau_{x y} \cos 2 \theta_{2} .
\end{aligned}
$$

From the equation system (6.17) it follows:

$$
\begin{aligned}
& \sigma_{y}=\tau_{2}\left(2 \cos 4 \theta_{2} \sin ^{2} \theta_{2}-\sin 4 \theta_{2} \sin 2 \theta_{2}-\cos 2 \theta_{2}-1\right)+2 \tau_{1} \\
& \tau_{x y}=-\tau_{2} \sin 4 \theta_{2}
\end{aligned}
$$

Since $\tau_{x y}=0$ along $y=0, x>0$ (smooth contact surface), substituting back into Eq. (6.18) $\theta_{2}$ can be determined. It follows from here that $\theta_{2}$ has the following solution:

$$
\theta_{2}=\frac{\pi}{2} \quad \text { for any } \lambda=\frac{\tau_{1}}{\tau_{2}}
$$


Then,

$$
\begin{aligned}
& \sigma_{y}=2\left(\tau_{1}+\tau_{2}\right), \\
& \left.\sigma_{x}=2 \tau_{1}, \quad \text { (in } \triangle \mathrm{ODE}\right) \\
& \tau_{x y}=0 .
\end{aligned}
$$

For the case of homogeneous material when $\tau_{1}=\tau_{2}$ from Eqs. (6.20) it follows:

$$
\begin{aligned}
& \sigma_{y}=4 \tau_{1}, \\
& \sigma_{x}=2 \tau_{1}, \quad \text { when } \theta_{2}=\frac{\pi}{2} \quad(\Delta \mathrm{ODE}) \\
& \tau_{x y}=0
\end{aligned}
$$

This solution again coincides with that found previously in Cherepanov [50] for this particular case.

From these results, it can be seen that this solution has a discontinuity in the stresses along the interface (line OC in Fig. 6.1). 


\subsection{Limiting Load}

The stresses found in this work, that are identical to those found previously in the case of a crack impinging an interface at a right angle, can be used to determine the limiting load or $P_{c r}$. This limiting load per unit of length is defined as:

$$
P_{c r}=\sigma_{y} L
$$

Where $\sigma_{y}$ is the normal stress in the $y$ direction in the region ODE, and $L$ is the length of contact of the punch (line OE in Fig. 6.1).

Using the solutions shown in this chapter, the limiting load for both solutions can be determined in the following form:

For the homogeneous case there are two possible solutions one is discontinuous and the other discontinuous.

a. Continuous case: From Eq. (6.11) it follows

$$
P_{c r}=\tau_{1}(2+\pi) L
$$


b. Discontinuous case: From Eq. (6.21) it follows

$$
P_{c r}=4 \tau_{1} L
$$

From Eqs. (6.23) and (6.24) it can be seen that $P_{c r}$ is greater for the case of the continuos solution than for the case of discontinuous solution.

For the inhomogeneous case, there is only one possible solution given by the discontinuous case. Substituting Eq. (6.20) into Eq. (6.22) it follows,

$$
P_{c r}=2\left(\tau_{1}+\tau_{2}\right) L=2 \tau_{2}(\lambda+1)
$$

Eq. (6.25) is valid for any $\lambda=\tau_{1} / \tau_{2}$.

\subsection{Velocity Distribution}

The velocity distribution is readily determined from the Geiringer equations. If the punch is moving with the velocity $V_{0}$ in the $y$ direction (Fig. 6.1), then region ODE moves as a rigid body attached to the punch with the same velocity. 
The results of simple calculations are given below:

1. The continuous solution:

$$
\begin{array}{lll}
v_{x}=0, & v_{y}=V_{0} & \text { in } \triangle \mathrm{ODE} \\
v_{r}=0, & v_{\theta}=\sqrt{2} V_{0} & \text { in } \triangle \mathrm{OBCD} \\
v_{x}=-V_{0}, & v_{y}=-V_{0} & \text { in } \triangle \mathrm{OAB}
\end{array}
$$

2. The discontinuous solution:

$$
\begin{array}{lll}
v_{x}=0, & v_{y}=V_{0} & \text { in } \triangle \mathrm{ODE} \\
v_{x}=0, & v_{y}=-V_{0} & \text { in } \triangle \mathrm{OABC}
\end{array}
$$




\section{CHAPTER 7}

\section{THE PUSH-IN AND PULL-OUT PROBLEMS}

\subsection{Introduction}

The push-in or pull-out problem has been considered extensively in order to understand the reinforcing effect of fibers in aligned composites. The mechanism of load transfer between the matrix and the reinforcement has been studied to determine the response of the composite under the action of axial loading such as that incurred by axial force at the top of the fiber during the push-in or pullout process.

In this chapter a previous result of an efficient solution of the pull-out problem developed by Cherepanov and Esparragoza (1995) is presented. Additional, the analysis of the stress state inside the plastic region at the crack tip during the push-in or pull-out process is considered. This analysis is useful to determine the critical load in this type of problem. Consequently, the type of mechanism of failure can be predicted using this analysis.

The push-in and pull-out problems are mathematically identical the only difference is the direction of the acting force. 


\subsection{Some Results of Fiber Stress Analysis}

In this section the main results of the study by Cherepanov and Esparragoza are summarized. For more details see the reference [51]. Consider a linearly elastic round cylinder embedded in a linearly elastic half-space of another material on a finite length (Fig. 7.1). The force, $F$, is applied to the open end of the fiber. The boundary of the half-space and lateral open surface of the cylinder is free of traction. Residual stresses are ignored in this work. Let us designate $r_{0}$ as the radius of the fiber, $l$, the length of the embedded part of the cylinder, $E_{f}$ and $E_{m}$ the Young's modulus of the fiber and matrix respectively, and $v_{f}$ and $v_{m}$ the Poisson's ratio of the fiber and matrix respectively. Introducing the following dimensionless quantities,

$$
\xi=\frac{r_{0}}{l}, \quad \varepsilon=\frac{E_{m}}{E_{f}}
$$

For sufficiently low force, $F$, there exists a perfect bonding between the fiber and the matrix, so that the no-slip and no-opening bonding conditions hold over all the interface including the lateral surface and the bottom. The problem is axisymmetric, so that $z$ is the axis of symmetry and $r$ is the radial distance from 
the axis. The case of most practical relevancy to composite when both $\xi$ and $\varepsilon$ are small is considered.

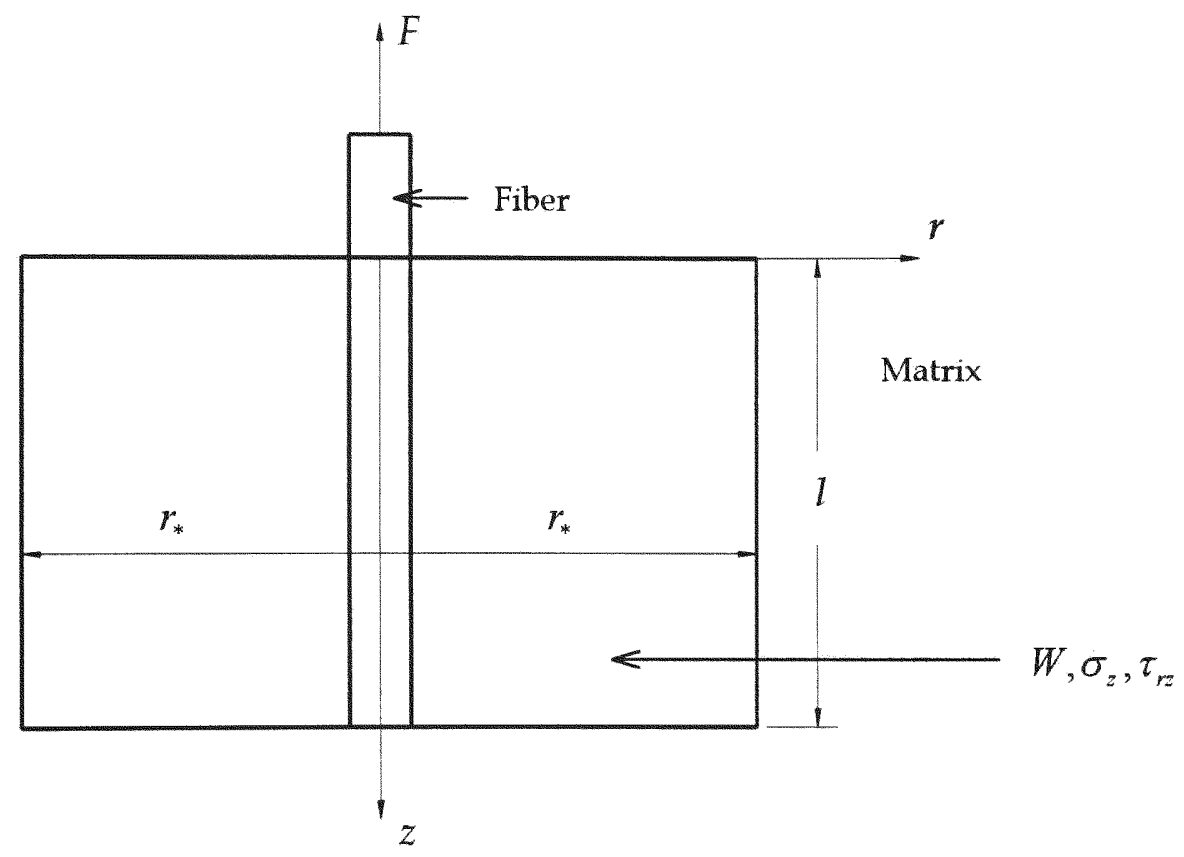

Fig. 7.1 The axisymmetric problem of pull-out of a foregoing elastic fiber embedded in a softer elastic matrix. The cylindrical domain of non-zero perturbed field.

In the case when $\xi<<1$ the stress field around the fiber has a structure of a boundary layer, in which the stress gradients across the fiber are much greater than along the fiber. Therefore, in this case the stress gradients with respect to $z$ can be neglected in the equilibrium equations as compared with those with 
respect to $x$ and $y$. As a result, a combination of anti-plane strain and plane strain equations are obtained.

The approximate analytical approach brought in Cherepanov (1983) is used. According to this approach the displacement and stress-strain field in the fiber are described by the mean normal stress, $\sigma_{z}(z)$, in a cross-section of the fiber, the shear stress, $\tau_{r z}(z)$, on the lateral surface of the fiber, and the mean displacement of the cross-section of the fiber $W$ along the $z$-axis. Following the same approach, the perturbed field of stresses, strains and displacements in matrix exist only inside the cylindrical domain $r<r_{*}, 0<z<l$ shown in Fig. 7.1. $r_{*}$ should be found from the following equation of fitting:

$$
\frac{r_{*}}{r_{0}}=\left(\frac{t}{r_{0}}\right)^{\eta}
$$

where $\eta$ is taken from Cherepanov (1983) as 0.738. Inside this cylindrical domain the fields of stress, strain, and displacement are described by the stress components of the stress tensor $\sigma_{z}=\sigma_{z}(r, z)$ and $\tau_{r z}=\tau_{r z}(r, z)$, and by the $z$ component of the displacement vector $w=w(r, z)$. All other components of the stress tensor and displacement vector are considered small enough to be ignored. 
Taking into account the equilibrium equation in the fiber and in the matrix, the boundary conditions and the assumptions described by Cherepanov (1983), an efficient analytical solution to the problem of pull-out or push-in is presented in the following form:

$$
\begin{aligned}
& \sigma_{z}=\frac{\sigma_{0}}{\sinh (\kappa)} \sinh \left[\kappa\left(1-\frac{z}{l}\right)\right] \\
& \tau_{r z}=\frac{\xi \kappa \sigma_{0}}{2 \sinh (\kappa)} \cosh \left[\kappa\left(1-\frac{z}{l}\right)\right] \\
& W=-\frac{\sigma_{0} l}{\kappa E_{f} \sinh (\kappa)} \cosh \left[\kappa\left(1-\frac{z}{l}\right)\right]
\end{aligned}
$$

Eqs. (7.3) to (7.5) are valid in the fiber. Here, $\kappa$ is the following expression,

$$
\kappa^{2}=\frac{1.355 \varepsilon}{\xi^{2}\left(1+v_{m}\right) \ln \left(\frac{1}{\xi}\right)}
$$

and

$$
\sigma_{0}=\frac{F}{\pi r_{0}^{2}}
$$


From Eqs. (7.3) and (7.4) it follows that the maximum stresses are:

$$
\begin{aligned}
\sigma_{\max } & =\sigma_{0} \\
\tau_{\max } & =\frac{\xi \kappa \sigma_{0}}{2} \frac{1}{\tanh (\kappa)} \quad \text { at } z=0 .
\end{aligned}
$$

The elastic pull-out problem is a necessary prelude to fiber debonding . The analytical solution for the pull-out or push-in problem for the partial debonding without friction can be derived in the form of Eqs. (7.3) to (7.5) replacing $z$ by $z-l_{D}$ and $l$ by $l-l_{D}$ in these equations, where $l_{D}$ is the length of the debonding zone of the fiber. In the case of debonding the following relation

$$
2 \tau_{s} \tanh \left(\kappa_{D}\right)=\xi_{D} \kappa_{D} \sigma_{0}
$$

holds between $\sigma_{0}, l_{D}$ and maximum interface shear stress, $\tau_{s}$, where $\xi_{D}$ and $\kappa_{D}$ correspond to $\xi$ and $\kappa$ when $l$ is replaced by $l-l_{D}$.

From the Eq. (7.8) it can be seen that when the length of debonding increases the relation $\sigma_{0} / \tau_{s}$ decreases. From here it can be concluded that after reaching the limiting shear stress along the interface, $\tau_{s}$, the debonding length appears and the crack start growing along the interface unstable. 
The analysis presented in this numeral covers the elastic problem and the debonding case for the case of an inhomogeneous material. The rest of this chapter deal with the situation when the plasticity is reached in the crack tip of two different elastic-perfectly plastic solids under the condition of plain strain subjected to the push-in or pull-out force.

\subsection{Statement of the Plasticity Problem}

It is assumed that the surface of the punch and body are perfectly lubricated, so that there is no friction between them (smooth surface line $\mathrm{OE}$ in Fig. 7.2). The constituent materials are also assumed elastic-perfectly plastic material and the theory of shear lines or slip lines is used in the same way that it was used in the previous chapters. In this case the stresses found here can be used to determine the limiting load.

Mathematically, the problem of stresses at the punch surface is identical to the boundary value problem of stresses at the crack front in plane strain. Consequently, Eqs. (4.1) to (4.5) are valid in this case. Surface OE in Fig. 7.2 is free of traction then $\sigma_{y}=\tau_{x y}=0$. 


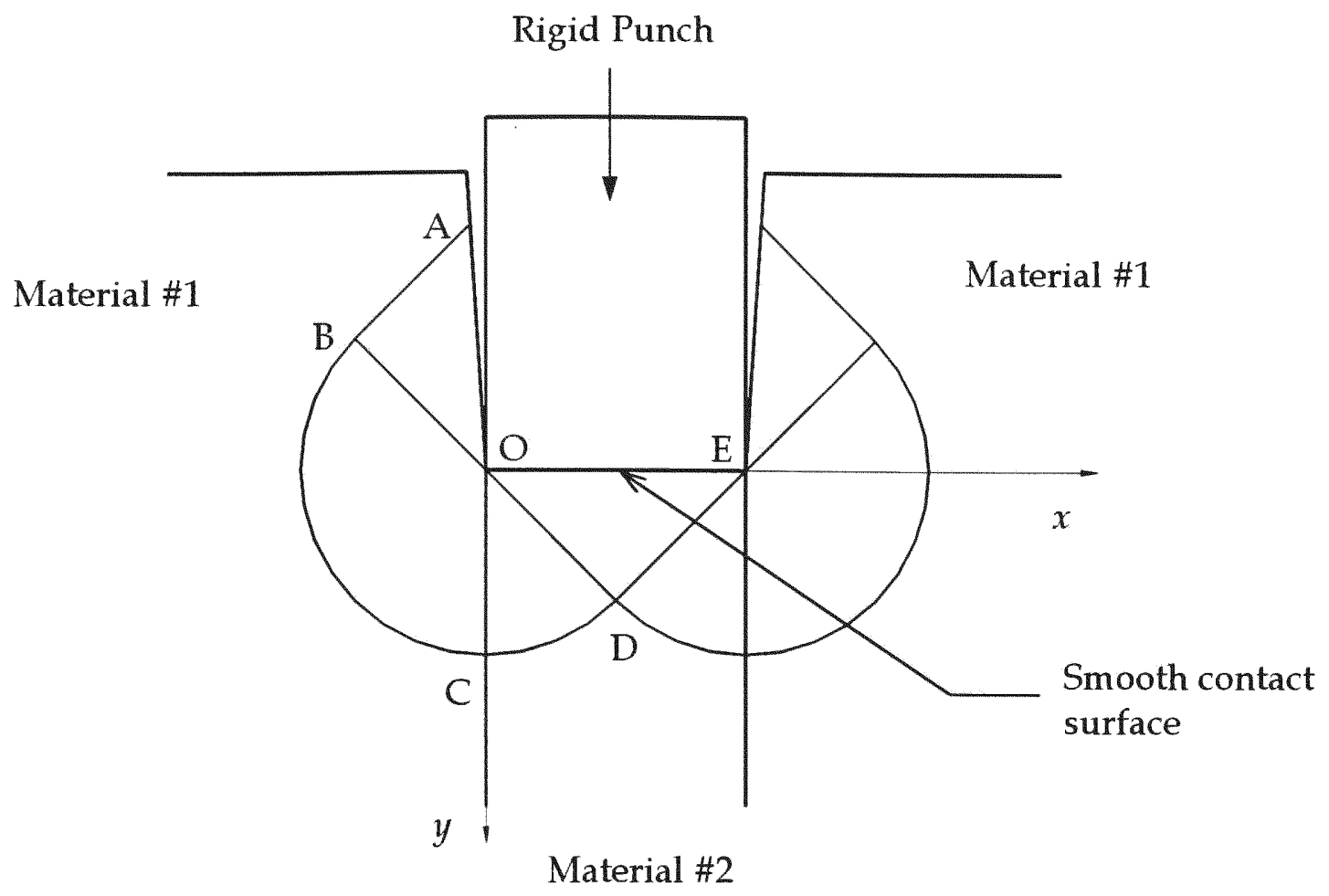

Fig. 7.2 The plasticity region in the push-in or pull out problem.

In region $O A B$ (Fig. 7.2) the only stress field is:

$$
\sigma_{x}=\tau_{x y}=0, \quad \sigma_{y}=2 \tau_{1} \quad \text { in } \quad \triangle \mathrm{OAB}
$$

Equilibrium and yielding condition equations in polar coordinates are the same of those expressed in Eqs. (4.7) to (4.9). 
Assuming the function $f(\theta)$ in the form of Eq. (4.10) and substituting back into (4.7) and (4.8) two possible solutions are also found for the stress field:

1. $\sigma_{r}=\sigma_{\theta}=\mp 2 \tau_{1} \theta+C_{3}, \quad \tau_{r \theta}= \pm \tau_{1}$

or

$$
\text { 2. } \begin{aligned}
\sigma_{r} & =\tau_{1} \sin \left(2 \theta+C_{1}\right)+C_{2}, \\
\sigma_{\theta} & =-\tau_{1} \sin \left(2 \theta+C_{1}\right)+C_{2}, \\
\tau_{r \theta} & =\tau_{1} \cos \left(2 \theta+C_{1}\right)
\end{aligned}
$$

\subsection{Continuous Solution}

According to Eq. (4.6), it follows:

$$
\sigma_{\theta}=\tau_{1}, \tau_{r \theta}=\tau_{1} \quad(\text { in } \Delta \mathrm{OAB})
$$

The stresses $\sigma_{\theta}$ and $\tau_{r \theta}$ should be continuous along OB. Then,

$$
\sigma_{\theta}=\tau_{1}, \quad \tau_{r \theta}=\tau_{1} \quad \text { along } \theta=\frac{5 \pi}{4}
$$


From Eqs. (4.15) and (7.13) it follows:

$$
\begin{aligned}
& \left.\sigma_{r}=\sigma_{\theta}=-2 \tau_{1} \theta+\tau_{1}\left(1+\frac{5 \pi}{2}\right) \quad \text { (in } \triangle \mathrm{OBC}\right) \\
& \tau_{r \theta}=\tau_{1}
\end{aligned}
$$

According to condition (4.5), the stresses $\sigma_{\theta}$ and $\tau_{r \theta}$ should be continuous along $\theta=\pi / 2(\mathrm{OC})$. This condition and the plasticity condition (Eq. (4.9) are met only for the homogeneous case when $\tau_{1}=\tau_{2}$,

$$
\begin{aligned}
& \sigma_{r}=-2 \tau_{1} \theta+\tau_{1}\left(1+\frac{5 \pi}{2}\right), \\
& \left.\sigma_{\theta}=-2 \tau_{1} \theta+\tau_{1}\left(1+\frac{5 \pi}{2}\right), \quad \text { (in } \triangle O C D\right) \\
& \tau_{r \theta}=\tau_{1}
\end{aligned}
$$

In the triangle ODE it follows from Eq. (4.3) for the homogeneous case:

$$
\sigma_{x}=\sigma_{y}-2 \sqrt{\tau_{1}^{2}-\tau_{x y}^{2}} \quad(\Delta \mathrm{ODE})
$$

Assumed here is that $\sigma_{y}>\sigma_{x}$ in this triangle which is physically evident for opening mode tensile cracks. 
The stresses, $\sigma_{\theta}$ and $\tau_{r \theta}$, should be continuous along OD. Designate $\theta=-\theta_{1}$ along OD. Expressing the stresses in the Cartesian coordinate system it follows that:

$$
\begin{aligned}
& -2 \tau_{1} \theta_{1}+\tau_{1}\left(1+\frac{5 \pi}{2}\right)=\left(\sigma_{y}-2 \sqrt{\tau_{1}^{2}-\tau_{x y}^{2}}\right) \sin ^{2} \theta_{1}+ \\
& +\sigma_{y} \cos ^{2} \theta_{1}-2 \tau_{x y} \sin \theta_{1} \cos \theta_{1} \\
& \tau_{1}=\sqrt{\tau_{1}^{2}-\tau_{x y}^{2}} \sin 2 \theta_{1}+\tau_{x y} \cos 2 \theta_{1}
\end{aligned}
$$

There are two equations and the condition of $\tau_{x y}=0$ along the smooth contact surface (line OE in Fig. 7.2 when $y=0$ and $x>0$ ) in order to determine $\theta_{1}$, and $\sigma_{y}$.

From Eq. (4.9) and the condition $\tau_{x y}=0, \theta_{1}$ can be found:

$$
\theta_{1}=\frac{\pi}{4}
$$

From the equation system (7.17) the continuous solution for the homogeneous case can be derived 


$$
\begin{array}{ll}
\sigma_{y} & =2 \tau_{1}(1+\pi), \\
\sigma_{x} & =2 \pi \tau_{1}, \\
\tau_{x y} & =0,
\end{array} \quad \text { when } \theta_{1}=\frac{\pi}{4}
$$

This is the continuous solution for the case of push-in or pull-out problem. Comparing Eqs. (6.11) and (7.19) it can be seen that the normal stresses in the region ODE are greater in the case of the push-in or pull-out problem than in the case of a smooth rigid punch.

\subsection{Discontinuous Solution}

According to Eq. (4.6), it follows:

$$
\sigma_{\theta}=\tau_{1}, \tau_{r \theta}=\tau_{1} \quad(\text { in } \triangle \mathrm{OAB})
$$

The stresses $\sigma_{\theta}$ and $\tau_{r \theta}$ should be continuous along $\mathrm{OB}$. Then,

$$
\sigma_{\theta}=\tau_{1}, \tau_{r \theta}=\tau_{1} \quad \text { along } \theta=\frac{5 \pi}{4}(\mathrm{OB})
$$


From Eqs. (4.16) and (7.21) it can be found:

$$
\begin{aligned}
& \sigma_{r}=\tau_{1}(1-\cos 2 \theta), \\
& \left.\sigma_{\theta}=\tau_{1}(1+\cos 2 \theta), \quad \text { (in } \triangle \mathrm{OBC}\right) \\
& \tau_{r \theta}=\tau_{1} \sin 2 \theta .
\end{aligned}
$$

According to condition (4.5), the stresses $\sigma_{\theta}$ and $\tau_{r \theta}$ should be continuous along $\theta=\pi / 2$ (OC). Then from Eqs. (4.5), (4.16) and (7.22) it follows:

$$
\begin{aligned}
& \sigma_{r}=\tau_{2}(1-\cos 2 \theta), \\
& \left.\sigma_{\theta}=\tau_{2}(1+\cos 2 \theta), \quad \text { (in } \triangle \mathrm{OCD}\right) \\
& \tau_{r \theta}=\tau_{2} \sin 2 \theta .
\end{aligned}
$$

In the triangle ODE it follows from Eq. (4.3):

$$
\sigma_{x}=\sigma_{y}-2 \sqrt{\tau_{2}^{2}-\tau_{x y}^{2}} \quad(\Delta \mathrm{ODE})
$$

The stresses, $\sigma_{\theta}$ and $\tau_{r \theta}$, should be continuous along $\mathrm{OD}$. Designate $\theta=\theta_{2}$ along OD. Expressing the stresses in the Cartesian coordinate system it follows that: 


$$
\begin{array}{ll}
\tau_{2}\left(1+\cos 2 \theta_{2}\right)=\left(\sigma_{y}-2 \sqrt{\tau_{2}^{2}-\tau_{x y}^{2}}\right) \sin ^{2} \theta_{2}+ & \\
+\sigma_{y} \cos ^{2} \theta_{2}-2 \tau_{x y} \sin \theta_{2} \cos \theta_{2} & \text { (in } \Delta \mathrm{ODE}) \\
\tau_{2} \sin 2 \theta_{2}=\sqrt{\tau_{2}^{2}-\tau_{x y}^{2}} \sin 2 \theta_{2}+\tau_{x y} \cos 2 \theta_{2} . &
\end{array}
$$

From the equation system (7.25) it follows:

$$
\begin{aligned}
\sigma_{y} & =\tau_{2}\left(1+\cos 2 \theta_{2}+2 \cos 4 \theta_{2} \sin ^{2} \theta_{2}+\sin 4 \theta_{2} \sin 2 \theta_{2}\right) \\
\tau_{x y} & =\tau_{2} \sin 4 \theta_{2}
\end{aligned}
$$

Since $\tau_{x y}=0$ along $y=0, x>0$ (smooth contact surface), substituting back into Eq. (7.26) $\theta_{2}$ can be determined. It follows from here that $\theta_{2}$ has the following solution:

$$
\theta_{2}=\frac{\pi}{2} \quad \text { for any } \lambda=\frac{\tau_{1}}{\tau_{2}}
$$

Then,

$$
\begin{aligned}
& \sigma_{y}=2 \tau_{2}, \\
& \sigma_{x}=0, \\
& \tau_{x y}=0 .
\end{aligned}
$$


For the case of homogeneous material when $\tau_{1}=\tau_{2}$ from Eqs. (7.28) it follows:

$$
\begin{aligned}
& \sigma_{y}=2 \tau_{1}, \\
& \sigma_{x}=0, \quad \text { when } \theta_{2}=\frac{\pi}{2} \quad(\triangle \mathrm{ODE}) \\
& \tau_{x y}=0
\end{aligned}
$$

This is the discontinuous solution of the stresses inside the plastic region for the push-in or pull-out problem. Comparing Eqs. (6.20) and (6.21) with Eqs. (7.28) and (7.29) it can be seen that the normal stresses in the region ODE are greater for the case of the smooth rigid punch than for the case of push-in or pull-out.

\subsection{Limiting Load}

The stresses components found for the continuous solution Eqs. (7.19) for the discontinuous case Eqs. (7.28) and (7.29) can be used to determine the limiting load per unit of length, $P_{c r}$, which is defined as:

$$
P_{c r}=\sigma_{y} L
$$

Where $\sigma_{y}$ is the normal stress in the $y$ direction in the region $\mathrm{ODE}$, and $L$ is the length of contact of the punch (line OE in Fig. 7.2). 
For the homogeneous case there are two possible solutions: one is discontinuous and the other discontinuous.

a. Continuous case: From Eq. (7.19) it follows

$$
P_{c r}=2 \tau_{1}(1+\pi) L
$$

b. Discontinuous case: From Eq. (7.29) it follows

$$
P_{c r}=2 \tau_{1} L
$$

From Eqs. (7.31) and (7.32) it can be seen that $P_{c r}$ is greater for the case of the continuos solution than that for the case of discontinuous solution.

For the inhomogeneous case, there is only one possible solution given by the discontinuous case. Substituting Eq. (7.28) into Eq. (7.30) it follows,

$$
P_{c r}=2 \tau_{2} L
$$

Eq. (7.33) is valid for any $\lambda=\tau_{1} / \tau_{2}$. 


\subsection{Velocity Distribution}

The velocity distribution is readily determined from the Geiringer equations. If the punch is moving with the velocity $V_{0}$ in the $y$ direction (Fig. 7.2), then region ODE moves as a rigid body attached to the punch with the same velocity.

The results of simple calculations are given below:

1. The continuous solution:

$$
\begin{array}{lll}
v_{x}=0, & v_{y}=V_{0} & \text { in } \triangle \mathrm{ODE} \\
v_{r}=0, & v_{\theta}=\sqrt{2} V_{0} & \text { in } \triangle \mathrm{OBCD} \\
v_{x}=V_{0}, & v_{y}=-V_{0} & \text { in } \triangle \mathrm{OAB}
\end{array}
$$

2. The discontinuous solution:

$$
\begin{array}{lll}
v_{x}=0, & v_{y}=V_{0} & \text { in } \triangle \mathrm{ODE} \\
v_{x}=0, & v_{y}=-V_{0} & \text { in } \triangle \mathrm{OCD} \\
v_{x}=0, & v_{y}=-V_{0} & \text { in } \triangle \mathrm{OCBA}
\end{array}
$$




\section{CHAPTER 8}

\section{DISCUSSION AND CONCLUSIONS}

\subsection{Introduction}

This dissertation has been devoted to the study of the stress state near the crack tip inside the plastic region for an inhomogeneous material made of two different elastic-perfectly plastic solids. Three different cases where considered: First, the case when the interface free of tractions was studied. Then, the case of the crack impinging the interface at a right angle was analyzed; finally, the case of the crack impinging the interface at arbitrary angle was considered. For any case both continuous and discontinuous solutions were presented based on the shear line or slip line theory.

Using the same idea by Hill and Sokolovsky who showed that the stresses at the crack tip coincides with those at the punch edge discovered by Prandtl for the case of a homogeneous material, this study was extended to the analysis of the stresses inside the plastic region when a rigid flat punch indents a semi-infinite inhomogeneous material. The same approach applies for the analysis of the push-in and pull-out problem. 
In this chapter, the discussion of the results as well as the conclusions and some recommendations for future work are treated.

\subsection{The Plasticity Problem at the Crack Tip for an Inhomogeneous Materials}

In this numeral the results of chapters 3 to 5 are discussed. General speaking, two different solutions have been found for each case: one is the continuous solution and the other is the discontinuous solution. It is necessary to determine which of these solutions is realized in practice because both of them cannot, evidently, be available simultaneously. So far, authors have been working with the continuous solutions (Prandtl's solution). However, the discontinuous solution cannot be thrown away without any reason.

For the homogeneous case it follows from Eqs. (3.26), (3.38), (4.27), (4.41) (5.15) and (5.26) that,

$$
\sigma_{y}=\tau_{1}(2+\pi), \quad \sigma_{x}=2 \pi, \quad \tau_{x y}=0 \quad \text { in } \triangle \mathrm{ODE}
$$

and

$$
\sigma_{y}=4 \tau_{1}, \quad \sigma_{x}=2 \tau_{1}, \quad \tau_{x y}=0 \quad \text { in } \triangle \mathrm{ODE}
$$


Where Eqs. (8.1) belong to the continuous solution while Eqs. (8.2) belong to discontinuous solution. There is a reason why the discontinuous solution is probably realized in practice, while the common continuos solution might not be met in practice. From the analysis of both solutions it can be seen that in the case of the homogeneous material the stresses $\sigma_{y}, \sigma_{x}$ in the wedge ODE for the continuos solution are greater than in the case of the discontinuous solution. Therefore, the material will yield first by the stresses of the discontinuous solution. Another reason comes from the comparison of both solutions for the punch problem: the discontinuous solution, with the discontinuity line issuing from the punch corner, seems physically more realistic than the smooth continuous solution by Prandtl. More sophisticated approaches based on the selection principle [53] (or the maximum energy dissipation principle [53]) also give the preference to the discontinuous solution. However, the final decision should be provided by experiments (probably, with pure cubic crystals best described by the elastic-perfectly plastic model).

In chapter 3 , in the case of a continuous solution the equation system (3.24)has a solution only for the case $0.71 \leq \lambda \leq 1$. Although different considerations were made to find a solution for the equation system (3.24), no solution was found in this work for $0.71 \geq \lambda \geq 0$. Experimental work is suggested to understand the behavior of the system under these circumstances. In this chapter it was also 
found that the discontinuous case has solution only for the limiting cases when $\lambda=1$ and when $\lambda=0$. The homogeneous case (when $\lambda=1$ ) was already analyzed. The case when $\lambda=0$ corresponds to a punch problem where the stresses in the wedge ODE (Fig. (3.1)) are those stated in Eq. (3.39).

In chapters 4 and 5 , the continuos case has a solution only for the case of a homogeneous material while the discontinuous solution is valid for any $\lambda$ from 0 to 1 . In the latter case, it is observed that the stress state in the plasticity region is formed only by constant areas and the discontinuity appears along the interface. It is expected from these results that the discontinuous case is the only possible solution and it might be realized in practice.

Using the found solutions in chapter 3 , the Reuss equations, and the equation of adhesion energy in the form of path-independent integral suggested by Cherepanov for a crack growth studies it can be shown that the adhesion energy is equal to zero in this model similar to the homogeneous case [53]. It means physically that the adhesion energy spent to initiate an interface crack in the elastic-perfectly plastic model is equal to zero, analogously to the homogeneous case [53], and the slow crack growth starts just after loading begins, and is accompanied by crack tip blunting. In the more accurate model the crack initiation based on the consideration of dislocation emission by a crack tip [54], it appears that the corresponding adhesion energy at the initial stage is non-zero, 
but very small (roughly ten times less that of an ideal crack growth with no dislocations emitted). In the well-developed crack growth range, it is necessary to take into account unloading and residual stresses and strains, which were not considered here. These circumstances do not allow efficient use of the elasticperfectly plastic model for calculation of adhesion energy characterizing the intermediate crack growth range, where the crack growth is comparable with the size of the plastic region near the crack tip.

\subsection{The Push-in and Pull-out Problem}

In the mechanics of push-in or pull-out, different situations can be considered. The case of a inhomogeneous surface indented by smooth rigid punch (chapter 6) is similar to the case of a push-in problem where one material is pushed-in into another material from the very surface. The case considered in chapter 7 numeral 7.3 implies that the process of push-in or pull-out has started already. Additionally, the elastic and debonding problem is also included in numeral 7.2.

First, the plasticity problem is analyzed. Since there are two different solutions one continuous and the other discontinuous, again it is necessary to determine which of these solutions is realized in practice because both cannot be available simultaneously. There are two reasons why the discontinuous solution is 
probably realized in practice while the common continuous solution might not be realized in practice. From chapter 6 Eqs. (6.23) and (6.24), it follows

$$
\begin{gathered}
P_{c r}=\tau_{1}(2+\pi) L \\
P_{c r}=4 \tau_{1} L
\end{gathered}
$$

and from chapter 7 Eqs. (7.31) and (7.32) it follows

$$
\begin{gathered}
P_{c r}=2 \tau_{1}(1+\pi) L \\
P_{c r}=2 \tau_{1} L
\end{gathered}
$$

Here Eqs. (8.3) and (8.5) belong to the continuous solution and Eqs. (8.4) and (8.6) belong to the discontinuous solution.

Therefore, the discontinuous solution provides a limiting load $P_{c r}$ smaller than that provided by the continuous solution in both the contact problem and the push-in and pull-out problem for the homogeneous case. Consequently, material will yield first by the limiting load $P_{c r}$ of the discontinuous solution. Secondly, the discontinuous solution provides a discontinuous displacement at the punch 
edge which is a more realistic profile of the local deformation than that suggested by the continuous solution giving a non-realistic smooth profile of local deformation near the punch ends.

For the case of an inhomogeneous material, only the discontinuous solution is available for any $\lambda$ and it might be realized in practice.

When an embedded material is subjected to a push-in or pull-out force, the following possible situations can be presented. First, the elastic behavior of the system occurs. In this case, the stresses along the embedded material under plain strain condition along the longitudinal direction are those expressed by Eqs. (7.3) and (7.4). Next, if the push-in or pull-out force is increased, then two things can happen. The maximum shear stress along the interface, Eq. (7.7), reaches the limiting shear stress at the interface that characterizes the ultimate strength of bonding forcing the bond to break. Then, the length of debonding starts growing but this growth is unstable, which means that the load decreases while the length of debonding grows. This behavior is predicted by Eq. (7.8) which shows that the normal stresses along the embedded material will decrease while the length of the debonding (the crack) grows. The other situation is that if the pushin or pull-out force is increased, the limiting load $P_{c r}$ can be reached. Therefore, the embedded material will yield and the Eq. (6.25) predicts this critical load. 
Comparing Eq. (6.25) with Eq. (7.33) it is observed that the case when the bonding interface holds along the whole interface (Fig. 6.1) produces a critical load, Eq. (6.25), greater than that for the case when the push-in or pull-out process started Eq. (7.33) (see Fig. 7.2). This result is consistent with the prediction of Eq. (7.8). The critical load to reach plasticity will be smaller for the case of push-in or pull-out Eq. (7.33) than that for the contact problem Eq. (6.25).

In any case, the debond zone tip is a singular point. Therefore, high stresses are expected at this point and plasticity zone will appear in the same form that for the crack tip. It means that once the limiting shear at the interface is reached and the debonding length appears a small plastic zone will develop at the debond tip and its size and distribution of stresses were already considered in this work.

\subsection{Final Remarks and Recommendations}

The stresses near the crack tip inside the plastic region for an inhomogeneous material made of two different elastic-perfectly plastic materials were studied for different positions of the crack with respect to the interface. In the corresponding homogeneous case two solutions were found one of which is the classical Prandtl's solution, and the other is a novel solution that was unnoticed by Prandtl, Hill ad Sokolovsky who earlier investigated this problem. 
The ideas were extended to the push-in and pull-out problem to provide more information to this classical problem in composite materials.

The study of a more sophisticated approach based on the selection principle (or the maximum energy dissipation principle) is also recommended and suggested as a complement work to this investigation to determine the preference of the discontinuous solution. However, the final decision should be provided by experiments (probably, with pure cubic crystals best described by the elasticperfectly plastic model). Then, it is also recommended to compare this results with experimental data. This information will be valuable to determine which solution is realized in practice and what happens in the case of an interface crack where there is still a range to cover for $0.71>\lambda \geq 0$.

Additional investigation related to this type of problem such as the case of a well-developed crack growth range taking into account unloading and residual stresses and strains, which were not considered here, is suggested as future research work.

The results of this dissertation published in three journal articles (two of them under revision [55], [56]) will be presented in the Invited Lecture on the $7^{\text {th }}$ International Conference on Plasticity (Cancun, Mexico, January (1999). 


\section{REFERENCES}

1. Tresca, H., 1864, Sur l'ecoulement des corps solides soumis à de fortes pression, C. R. Acad. Sci. Paris, 59, 754.

2. Saint-Venant, B., 1870, Memorie sur l'establissement des équations différentielles des mouvements intérieurs opéres dans les corps solides ductiles au delà des limites où l'élasticité pourrait les ramener à leur premier état, C. R. Acad. Paris, 70, 473.

3. Levy, M., 1870, Momoire sur les équations générales des mouvements intérieurs des corps solids ductiles au delà des limites où l'élasticité pourrait les ramener à leur premier état, C. R. Acad. Paris, 70, 1323.

4. Haar, A. and von Karman, T., 1909, Zur Theorie der Spannungszustände in plastichen und sandartigen Medien, Nachr. Ges. Wiss. Göttingen, 204.

5. Huber, M. T., 1904, Czasopismo techniczne, Lemberg, 22, 181.

6. Von Mises, R., 1913, Mechanik der fasten Körper im plastisch deformablen Zustant, Nachr. Ges. Wiss. Göttingen, 582.

7. Hencky, H., 1924, Zur Theorie Plasticher Deformationen und der hierduch im Material hervorgerufenen Nebenspannunger, Z. angew. Math. Mech., 4, 323.

8. Prandtl, L., 1920, Über die Härte Plasticher Körper, Nachr. Ges. Wiss. Göttingen, 74 .

9. Hencky, H., 1923, Über einige statisch bestimmte Fälle des Gleichgewitchts in plastichen Körpern, Z. angew. Math. Mech., 3, 241.

10. Geiringer, H., 1930, Beitrag zur Vollständigen ebenen Plastizitäts problem, Proc. $3^{\text {rd }}$ Intern. Congr. App. Mech., 2, 185.

11. Hill, R., 1950, The Mathematical Theory of Plasticity, Oxford University Press, London.

12. Sokolovsky, V.V., 1950, Theory of Plasticity, Gostekhizdat, Moscow. 
13. Prager, W., and Hodge, P. G., 1951, Theory of Perfectly Solids, Wiley, New York.

14. Green, A. P., 1951, A theoretical investigation of the compression of a ductile material between smooth flat dies, Phil. Mag., 42, 900.

15. Green, A. P., 1953, Plastic Yielding of notched bars due to bending, Q. $J l$. Mech. Appl. Math., 6, 223.

16. Green, A. P., 1954, On symmetrical extrusion in plane strain, J. Mech. Phys. Solids., 3, 189.

17. Prager, W., 1953, A geometrical discussion of the slip line field in plane plastic flow, Trans. R. Inst. Technol., Stockholm, 65, 27.

18. Alexander, J. M., 1955, The effect of Coulomb friction in the plane strain compression of a plastic-rigid material, J. Mech. Phys. Solids, 3, 233.

19. Johnson, W., 1956, Extrusion through square dies of large reduction, J. Mech. Phys. Solids, 4, 191.

20. Griffith, A. A., 1921, The phenomena of rupture and flow in solids, Phil. Trans. Roy. Soc. of London, A21, 163.

21. Muskhelishvili, N. I., 1933, Some basic problems of mathematical theory of elsticity. English translation, Noordhoff, 1953.

22. Westergaard, H. M., 1939, Bearing pressures and cracks, J. Appl. Mech., 61, A49.

23. Irwin, G. R., 1948, Fracture dynamics, Fracturing of Metals, ASM publ. 147.

24. Irwin, G. R., 1958, Fracture, Handbuch der Physic VI, Flügge Ed., Springer, 551.

25. Irwin, G. R., 1960, Plastic zone near a crack and fracture toughness,Proc. $7^{\text {th }}$ Sagamore Conf., IV-63.

26. Dugdale, D. S., 1960, Yielding of steel sheets containing slits, J. Mech. Phys. Sol., 8, 100 .

27. Wells, A. A., 1961, Unstable crack propagation in metals, cleavage and fast fracture, The crack propagation Symposium, Cranfield, 210. 
28. Cherepanov, G. P., 1967, Crack growth in continuous media, PMM, Vol 41, 3, 399. (in Russian). English translation in FRACTURE: Topical Encyclopedia of Current Knowledge, Krieger Publ., 1998, Chapter 3.

29. Rice, J. R., 1968, A path independent integral and the approximate analysis of strain concentrations by notches and cracks, J. Appl. Mech., 35, 379-386.

30. Hutchinson, J. W., 1968, Singular behavior at the end of a tensile crack tip in a hardening material, J. Mech. Phys. Sol., Vol. 16, 33.

31. Hutchinson, J. W., 1968, Plastic stress and strain fields at the crack tip, J. Mech. Phys. Sol., Vol. 16, 337.

32. Rice, J. R., and Rosengren, G. F., 1968, Plane strain deformation near a crack tip in a power-law hardening material, J. Mech. Phys. Sol., Vol. 16, 1.

33. Kahl, M., and Reifsnider, K. L., 1972, Influence of local variations of yield strength on plastic zones at crack tips, Engr. Fract. Mech., Vol. 4, 653-664.

34. Goldman, N. L., and Hutchinson, J. W., 1975, Fully plastic crack problems: the center-cracked strip under plane strain, Int. J. Sol. Structures, Vol. 11, 575591.

35. Amazigo, J. C., 1975, Fully plastic center-cracked strip under anti-plane shear, Int. J. Sol. Structures, Vol. 11, 1291-1299.

36. Amazigo, J. C., 1978, Some mathematical problems of elastic-plastic crack growth, presented at the AMS/SIAM Symposium on Mathematical Problems in Fracture Mechanics.

37. Shih, C. F., and Hutchinson, J. W., 1976, Fully plastic solutions and large scale yielding estimates for plane stress crack problems, J. Eng. Mat. Tech., ASME, Series H., Vol. 98, 289-295.

38. Shih, C. F., 1976, J Integral estimates for strain hardening materials in antiplane shear using fully plastic solutions, in Mechanics of Crack Growth, ASME STP 590, 3-22.

39. McMeeking, R. M., and Parks, D. M., 1977, On criteria for J-Dominance of crack tip fields, presented at the ASTM Symposium on Elastic-Plastic Fracture, Atlanta. 
40. Zahoor, A., and Paris, P. C., 1978, Instability analysis of a fully plastic centercracked strip of a power hardening material, Mechanical Engineering Department, Washington University.

41. Zahoor, A., and Paris, P. C., 1978, General instability analysis of a centercracked strip of an elastic-plastic strain hardening material, Mechanical Engineerin Department, Washington University.

42. Rice, J. R., 1978, Some mathematical problems of elastic-plastic crack growth, presented at the AMS/SIAM Symposium on Mathematical Problems in Fracture Mechanics.

43. Marcal, P. V., 1969, Finite element analysis with material nonlinearitiesTheory and practice, Brown University.

44. Adams, D. F., 1970, Inelastic analysis of a unidirectionla composite subjected to transverse normal loading, Rand Corp. Rep., RM-6245-PR.

45. Bert, C. W., 1972, Plasticity and creep analysis of filmentary metal-matrix composites, Sandia Corp. Rep., SC-DR-720055.

46. Repnau, T., and Adams, D. F., 1973, A finite element computer program for the elastoplastic analysis of crack propagation in a unidirectional composite. Rand Corp. Rep., R-1392-PR.

47. Nair, P, and Reifsnider, K. L., 1974, Unsymmetric deformartion fields in symmetric-geometry specimens with non-uniform properties, Proc. Southeastern Conf. On Theoretical and Applied Mechanics, Vol. 7, Catholic University, Washington, 183-196.

48. Reifsnider, K. L., Henneke, E. G., and Stinchcomb, W. W., 1976-77, Defect property relationships in composite materials, AFML-Technical Report, 7681.

49. Reifsnider, K. L., 1977, Some fundamentals aspects of the fatigue and fracture response of composite materials, Proc. Of $14^{\text {th }}$ Annual Meeting of the Society of Engineering Science, Lehigh University Press.

50. Cherepanov, G. P., 1997, Methods of Fracture Mechanics. Solid Matter Physics. Kluwer, Academic Publishers, Dordrecht. 
51. Cherepanov, G. P. and Esparragoza, I. E., 1995, The pullout problem, Mat. Science E Eng., A203, 332-342.

52. Cherepanov, G. P., 1983, Fracture Mechanics of Composite Materials, Moscow, Nauka Publisher, 308, (in Russian).

53. Cherepanov, G. P., 1974, Mekhanika Khrupkogo Razrushemia, Moscow, Nauka; English Edition, Mechanics of Brittle Fracture, Edited by R. de Wit and W. C. Cooley, McGraw Hill, New York, 1979.

54. Cherepanov, G. P., Richter, A., Verijenko, V. E., Adali, S., and Sutyrin, V., 1995, Dislocation generation and crack growth under monotonic loading, J. of Appl. Phys., Vol. 78, No. 10, 6249-6264.

55. Esparragoza, I. E., An interface crack between two different elastic-plastic solids, Under revision from Int. J. of Plasticity, 1997.

56. Esparragoza, I. E., The crack impinging an interface of two different elasticplastic solids under the right angle, Submitted to J. of Comp. Mat., 1998.

57. Esparragoza, I. E., The crack impinging an interface of two different elasticplastic solids under an arbitrary angle, Under preparation, 1998.

58. Cherepanov, G.P., and Esparragoza, I. E., Crack tip plasticity, Invited Lecture on the $7^{\text {th }}$ International Conference on Plasticity, Cancun, Mexico, January 1999. 
July 17, 1964

EDUCATION

1987

1993

1998

1985-1986

1985-1987

1988

1989-1991

EXPERIENCE
Born, Barranquilla, Colombia

B. Sc., Mechanical Engineering

Universidad Del Norte

Barranquilla, Colombia

M. Sc., Mechanical Engineering Florida International University

Miami, Florida

Ph.D., Mechanical Engineering Florida International University

Miami, Florida

Teaching Assistant

Universidad Del Norte

Barranquilla, Colombia

Chief Engineer

Ministerio de Comunicaciones

Barranquilla, Colombia

Lecturer

Universidad Del Norte

Barranquilla, Colombia

Superintendent

Aserradero Covadonga S.A.

Barranquilla, Colombia 
1992-1998

Research Assistant

Florida International University

Miami, Florida

1996-1998

Instructor

Florida International University

Miami, Florida

\section{PUBLICATIONS AND PRESENTATIONS}

Esparragoza, E. A., and Esparragoza, I. E., 1987, Diseño, construcción y montaje de un banco didáctico para determinar los parámetros básicos de ignición, arranque, carga y regulación de un motor a gasolina, Universidad Del Norte, Barranquilla, Colombia, (in Spanish).

Esparragoza, I. E., 1993, Numerical Analysis of the Pullout Problem of a Fiber Embedded in a Matrix: Comparison with an Approximate Analytical Solution, Florida International University, Miami, Fl.

Cherepanov, G. P., and Esparragoza, I. E., 1995, The Problem of Pullout, Materials Science and Engineering, A203, 332-342.

Esparragoza, I. E., An interface crack between two different elastic-plastic solids, Under revision International Journal of Plasticity, 1997.

Esparragoza, I. E., The crack impinging an interface of two different elasticplastic solids under the right angle, Submitted to Joumal of Composite Materials, 1998.

Esparragoza I. E., The crack impinging the interface of two different elasticplastic solids al an arbitrary angle, Under preparation, 1998.

Cherepanov, G. P., and Esparragoza, I. E., Crack tip plasticity, Invited Lecture on the $7^{\text {th }}$ Symposium of Plasticity, Cancun, Mexico, 1999. 